\title{
Gravitational spin-orbit coupling in binary systems at the second post-Minkowskian approximation
}

\author{
Donato Bini ${ }^{1}$ and Thibault Damour ${ }^{2}$ \\ ${ }^{1}$ Istituto per le Applicazioni del Calcolo "M. Picone", CNR, I-00185 Rome, Italy \\ ${ }^{2}$ Institut des Hautes Etudes Scientifiques, 91440 Bures-sur-Yvette, France
}

(Dated: May 29, 2018)

\begin{abstract}
We compute the rotations, during a scattering encounter, of the spins of two gravitationally interacting particles at second-order in the gravitational constant (second post-Minkowskian order). Following a strategy introduced in Phys. Rev. D 96, 104038 (2017), we transcribe our result into a correspondingly improved knowledge of the spin-orbit sector of the Effective One-Body (EOB) Hamiltonian description of the dynamics of spinning binary systems. We indicate ways of resumming our results for defining improved versions of spinning EOB codes which might help in providing a better analytical description of the dynamics of coalescing spinning binary black holes.
\end{abstract}

\section{INTRODUCTION}

The Effective One-Body (EOB) formalism [1] $[4]$ is a theoretical framework which yields a resummed analytical description of the gravitational dynamics of two spinning bodies. It can be essentially thought as mapping the dynamics (in the center-of-mass frame) of two bodies, of masses $m_{1}, m_{2}$ and spins $\mathbf{S}_{1}, \mathbf{S}_{2}$, onto a Hamiltonian description of the motion of a single body, of mass

$$
\mu \equiv \frac{m_{1} m_{2}}{m_{1}+m_{2}}
$$

and (suitably rescaled) spin

$$
\mathbf{S}_{*} \equiv \frac{m_{2}}{m_{1}} \mathbf{S}_{1}+\frac{m_{1}}{m_{2}} \mathbf{S}_{2},
$$

moving (modulo some higher-in-momenta corrections, and modified spin-orbit effects) in an effective metric that is a deformed version of a Kerr metric of mass

$$
M \equiv m_{1}+m_{2},
$$

and spin $\mathbf{S}_{0}=\mathbf{S}+\mathbf{S}_{*}$, where

$$
\mathbf{S} \equiv \mathbf{S}_{1}+\mathbf{S}_{2} .
$$

The key deformation parameter entering EOB theory is the symmetric mass ratio

$$
\nu \equiv \frac{\mu}{M}=\frac{m_{1} m_{2}}{\left(m_{1}+m_{2}\right)^{2}} .
$$

Since its introduction about twenty years ago [1-4] the EOB approach has been continuously improved by incorporating new relevant results obtained through various formalisms, such as Post-Newtonian (PN) theory, Numerical Relativity (NR), Gravitational Self-Force (GSF) theory, and, more recently Post-Minkowskian (PM) theory, and even Quantum Scattering Amplitudes. The EOB formalism has been the basis of the computation of many of the gravitational wave templates [5, 6] which have been used in the data analysis of the gravitational wave signals detected by the LIGO and Virgo interferometers $7-10$.
From the practical point of view, the EOB Hamiltonian is made of several building blocks involving various coupling functions or "potentials." The EOB potentials were initially computed in PN-expanded form, i.e. as power series in the inverse of the speed of light, $\frac{1}{c}$. This $\mathrm{PN}$-expanded approach has, however, several limitations. It, indeed, becomes inaccurate in two regimes that are relevant for theoretically describing present and future gravitational-wave observations, namely: (i) when two bodies of comparable masses become very close to each other, and also, (ii) when a small body moves on a highenergy orbit around a large one. The first limitation has been tamed by a combination of analytical resummation methods [3, 11] and of strong-field improvements of the EOB potentials by best fitting them to the results of a few NR simulations [12 14]. The second limitation has been shown to give rise to power-law singularities, at a finite radius corresponding to the light-ring, in the small$\nu$ expansion of the EOB potentials (as they are usually defined) [15].

The latter (light-ring related) limitation was, however, shown to be due to the combined use of a GSF expansion in powers of $\nu$, Eq. (1.5), and of a special choice of coordinates in phase-space. By using another way of fixing the phase-space coordinates, and by using the exact dependence of the resulting EOB potentials on $\nu$, one can avoid the presence of a singularity at the light ring [15, 16]. In order to reach such a conclusion, it was necessary to use a Post-Minkowskian (PM) approach to EOB theory [17], i.e. an expansion in powers of the gravitational constant $G$, without using an expansion in $\frac{1}{c}$ (i.e. without making any slow-motion assumption).

After its introduction in Ref. [17], the PM approach to EOB theory was extended to spin coupling effects in Ref. 18]. The spin-orbit contribution to the EOB effective Hamiltonian is parametrized by two gyrogravitomagnetic ratios, $g_{S}$ and $g_{S_{*}}$ :

$$
\begin{aligned}
H_{\mathrm{eff}} & =\sqrt{A\left(\mu^{2}+\mathbf{P}^{2}+\left(\frac{1}{B}-1\right) P_{R}^{2}+Q\right)} \\
& +\frac{G}{R^{3}}\left(g_{S} \mathbf{L} \cdot \mathbf{S}+g_{S_{*}} \mathbf{L} \cdot \mathbf{S}_{*}\right)
\end{aligned}
$$


where $\mathbf{S}$ and $\mathbf{S}_{*}$ have been defined in Eqs. (1.4), (1.2), above, and where $\mathbf{L}$ denotes the EOB orbital angular momentum

$$
\mathbf{L}=\mathbf{R} \times \mathbf{P}
$$

Here, and below, we use standard vectorial notation for various EOB vectorlike objects.

The values of $g_{S}$ and $g_{S_{*}}$ have been computed in Ref. 18] at the first post-Minkowskian (1PM) order (i.e., firstorder in $G$ but all orders in $v / c$ ). [In view of the factorization of one power of $G$ in the definition of the spin-orbit contribution to Eq. (1.6), this meant computing $g_{S}$ and $g_{S_{*}}$ as exact functions of the energy, at zeroth order in the gravitational potential $u \equiv G M /\left(c^{2} R\right)$.] For similar PM results for nonlinear effects in the spins, see Ref. [19].

As explained in Ref. [18, the PM computation of spinorbit couplings is achieved by considering the spatial rotations of the spin vectors of the two gravitationally interacting bodies during the full scattering process of an hyperboliclike encounter. The latter spatial rotations are the spin analogs of the orbital scattering angle, and are measured by the "spin holonomy" [18], whose definition is recalled below.

The aim of the present paper is to extend the accuracy of the computation of the spin holonomy to the second post-Minkowskian (2PM) level, and to deduce from it the 2PM-accurate values of the two EOB gyrogravitomagnetic couplings, i.e.

$$
\begin{aligned}
g_{S} & =g_{S}^{1 \mathrm{PM}}\left(H_{\mathrm{eff}}\right)+g_{S}^{2 \mathrm{PM}}\left(H_{\mathrm{eff}}\right) u+O\left(u^{2}\right), \\
g_{S *} & =g_{S *}^{1 \mathrm{PM}}\left(H_{\mathrm{eff}}\right)+g_{S *}^{2 \mathrm{PM}}\left(H_{\mathrm{eff}}\right) u+O\left(u^{2}\right),
\end{aligned}
$$

where we recall that $u \equiv G M /\left(c^{2} R\right)=O(G)$. Our 2PMlevel computation will make a crucial use of the 2PMaccurate explicit analytic computation of the metric generated by two (non-spinning) bodies done long ago by Bel et al. [20].

We use a mostly plus spacetime signature, and will often use units where $c=1$. The spin four-vectors of the two bodies are denoted by $s_{1}$ and $s_{2}$. The latter fourvectors are (when working, as we do, linearly in spins) orthogonal to the worldlines of the bodies. The EOB spin three-vectors $\mathbf{S}_{1}, \mathbf{S}_{2}$ boatin obtained by boosting $s_{1}$ and $s_{2}$ to the center-of-mass frame. [Viewed in the asymptotically flat spacetime the boosting of the fourvectors $s_{1}$ and $s_{2}$ defines corresponding spacetime fourvectors $S_{1}$ and $S_{2}$, whose (only non vanishing) spatial components define the EOB spin vectors $\mathbf{S}_{1}$ and $\mathbf{S}_{2}$.]

\section{SCATTERING HOLONOMY}

We consider the gravitational scattering of two massive and spinning bodies at linear order in spin. At this order, we can compute the spin holonomy simply from the knowledge of the metric $g\left(m_{1}, m_{2}\right)=$ $g_{\mu \nu} d x^{\mu} \otimes d x^{\nu}$ generated by two non spinning masses $m_{1}$, $m_{2}$. In addition, we can neglect the spin-curvature force present in the Mathisson-Papapetrou-Dixon equations, i.e. consider that each spin vector is parallely propagated in $g_{\mu \nu}\left(m_{1}, m_{2}\right)$. The explicit value of the metric $g_{\mu \nu}\left(m_{1}, m_{2}\right)$ generated by the energy-momentum tensor associated with the two masses $m_{1}, m_{2}$ has been explicitly computed at the $2 \mathrm{PM}$ level of approximation in Ref. 20]. [We shall not use here the less explicit 2PM-level formulation of the 2PM-accurate metric in Ref. [21].]

The worldline of body 1 (mass $m_{1}$ and spin $s_{1}$ ) is $\mathcal{L}_{1}$ with $g$-normalized timelike, future-pointing, tangent vector $u_{1}\left(u_{1} \cdot g u_{1} \equiv g_{\alpha \beta} u_{1}^{\alpha} u_{1}^{\beta}=-1\right)$ and linear momentum $p_{1}=m_{1} u_{1}\left(p_{1} \cdot g p_{1}=-m_{1}^{2}\right)$. The four-velocity $u_{1}$ satisfies the geodesic equation

$$
\frac{D_{g} u_{1}}{d \tau_{1}^{g}}=0
$$

where $\tau_{1}^{g}$ is the proper-time parametrization for $\mathcal{L}_{1}$ and $D_{g}$ is the covariant derivative along $u_{1}$, both associated with the metric $g$. The spin vector $s_{1}$ is constrained by the condition of being orthogonal to the linear momentum $p_{1}$

$$
s_{1} \cdot g p_{1}=0,
$$

and evolves along $\mathcal{L}_{1}$ by parallel transport

$$
\frac{D_{g} s_{1}}{d \tau_{1}^{g}}=0
$$

As a consequence, the magnitude of $s_{1}$ is conserved, and the orthogonality condition (2.2) is preserved. Corresponding equivalent statements can be done for $u_{2}$ and $s_{2}$ and their evolution along $\mathcal{L}_{2}$.

Using abstract notation and differential forms, the transport equations can be recast as

$$
D_{g} u_{1}=0=D_{g} s_{1}
$$

Here

$$
D_{g}=d+\omega_{1}
$$

where $d$ denotes the ordinary differential operation along $\mathcal{L}_{1}$ while $\omega_{1}$ denotes the evaluation along $\mathcal{L}_{1}$ of the LeviCivita connection one-form, $\omega$. In a coordinate frame the connection one-form acting on contravariant four-vectors is given by

$$
\omega_{\nu}^{\mu}=\Gamma_{\nu \lambda}^{\mu} d x^{\lambda}
$$

where

$$
\Gamma_{\nu \lambda}^{\mu}=\frac{1}{2} g^{\mu \sigma}\left(\partial_{\nu} g_{\lambda \sigma}+\partial_{\lambda} g_{\nu \sigma}-\partial_{\sigma} g_{\nu \lambda}\right) .
$$

When dealing, as we do here, with the metric generated by point masses, the metric and the connection are singular when evaluated on the worldlines. We will use their regularized values, as explicitly discussed and computed in Ref. [20]. 
Let us briefly recall the two basic concepts of scattering holonomy and spin holonomy, introduced in Ref. [18]. First, the scattering holonomy along the worldline $\mathcal{L}_{1}$ is the linear operator $\Lambda_{1}$ of integrated parallel-transport, acting on contravariant vectors. The integration along $\mathcal{L}_{1}$ is performed from the infinite past (i.e., before the interaction, where the spacetime is assumed to be flat) to the infinite future (i.e., after the interaction where again the spacetime is assumed to be flat, since we are studying here an isolated system). The two asymptotic Minkowski flat spacetimes are (at this order) unambiguously identified among them, so that $\Lambda_{1}=\Lambda_{1 \nu}^{\mu}$ is simply a Lorentz matrix (preserving the Poincaré-Minkowski metric $\eta_{\mu \nu}$ ).

The solution of the evolution equation of a parallely transported vector $v$

$$
d v=-\omega_{1} v
$$

can be iteratively solved as

$$
\begin{aligned}
v^{\mu}(\tau)= & v^{\mu}(-\infty)-\int_{-\infty}^{\tau} \omega_{1 \nu}^{\mu}(\tau) v^{\nu}(\tau) \\
= & v^{\mu}(-\infty)-\int_{-\infty}^{\tau} \omega_{1 \nu}^{\mu}(\tau)\left[v^{\nu}(-\infty)\right. \\
& \left.\quad-\int_{-\infty}^{\tau} \omega_{1 \sigma}^{\nu}\left(\tau^{\prime}\right) v^{\sigma}\left(\tau^{\prime}\right)\right]+\ldots
\end{aligned}
$$

so that when $\tau \rightarrow+\infty$ we find

$$
v_{+}^{\mu}=\Lambda_{1 \nu}^{\mu} v_{-}^{\nu},
$$

where $v^{\mu}( \pm \infty)=v_{ \pm}^{\mu}$. The scattering holonomy operator $\Lambda_{1}$ is therefore given by

$$
\begin{aligned}
\Lambda_{1} & =T_{\mathcal{L}_{1}}\left[e^{-\int \omega_{1}}\right] \\
& =I-\int_{-\infty}^{+\infty} \omega_{1}+\frac{1}{2} \int_{-\infty}^{+\infty} \int_{-\infty}^{+\infty} T\left[\omega_{1} \omega_{1}^{\prime}\right]+
\end{aligned}
$$

with $T_{\mathcal{L}_{1}}$ denoting Dyson's time-ordered product 22] along the worldline $\mathcal{L}_{1}$. The $T$-ordered integral is such that

$$
\frac{1}{2} \iint T_{\mathcal{L}_{1}}\left[\omega_{1} \omega_{1}^{\prime}\right]^{\mu}{ }_{\nu}=\int_{-\infty}^{+\infty} \omega_{1 \sigma}^{\mu}(\tau) \int_{-\infty}^{\tau} \omega_{1}^{\sigma}{ }_{\nu}\left(\tau^{\prime}\right) .
$$

[Note that in our differential-form formulation, $\omega_{1 \nu}^{\mu}(\tau)=$ $\Omega_{1 \nu}^{\mu}(\tau) d \tau$, the integration does not depend on the choice of any specific parametrization $\tau$ along the worldline.] The scattering holonomy map $\Lambda_{1}$ is then a linear map relating the asymptotic states $u_{1}^{-}, s_{1}^{-}$and $u_{1}^{+}, s_{1}^{+}$:

$$
u_{1}^{+}=\Lambda_{1} u_{1}^{-} ; \quad s_{1}^{+}=\Lambda_{1} s_{1}^{-} .
$$

The linear map $\Lambda_{1}$ contains information both about the orbital scattering angle, and the spin-rotation angle, of the first particle. Some extra operation is needed to extract the spin rotation of direct interest for computing the EOB spin-orbit coupling entering the effective
EOB Hamiltonian (1.6). What is needed is an operation transforming the covariant spin four-vectors $s_{1}, s_{2}$ into objects directly related to the spatial, canonical spin three-vectors $\mathbf{S}_{1}, \mathbf{S}_{2}$ that enter any Hamiltonian dynamics, like the EOB dynamics. As discussed in Ref. [18], $\mathbf{S}_{1}$ and $\mathbf{S}_{2}$ can be identified with the four-vectors $S_{1}, S_{2}$ defined by boosting $s_{1}$ and $s_{2}$, respectively, from their own local rest spaces ( $s_{1}$ is orthogonal to $u_{1}$ and $s_{2}$ is orthogonal to $u_{2}$ ) onto the local three-space defined by a global field of (future-directed) unit time-vectors $U=U^{\mu} e_{\mu}\left(e_{\mu}\right.$ denoting here a coordinate frame) orthogonal to the timeslicing used to describe the full spacetime generated by the two particles. In a scattering problem, this global field only enters through its asymptotic values at $\pm \infty$, $U=U^{\text {as }}$. The corresponding asymptotic values of the spin vector $S_{1}$ are then given by

$$
\begin{aligned}
& S_{1}^{+}=B_{\eta}\left(u_{1}^{+} \rightarrow U^{\mathrm{as}}\right) s_{1}^{+} \\
& S_{1}^{-}=B_{\eta}\left(u_{1}^{-} \rightarrow U^{\mathrm{as}}\right) s_{1}^{-},
\end{aligned}
$$

with $\eta$ being the Minkowski metric at $\pm \infty$. Here, the linear map $B_{\eta}(u \rightarrow v)$ is the Lorentz boost matrix transforming the four-vector $u$ into the four-vector $v$. The explicit expression of the Lorentz matrix $\left[B_{\eta}(u \rightarrow v)\right]^{\mu}{ }_{\nu}$ is (see, e.g., [18])

$$
\begin{aligned}
& {\left[B_{\eta}(u \rightarrow v)\right]_{\nu}^{\mu}=\delta_{\nu}^{\mu} } \\
+ & \frac{1}{1-u \cdot v}\left[u^{\mu} u_{\nu}+v^{\mu} v_{\nu}+u^{\mu} v_{\nu}-(1-2 u \cdot v) v^{\mu} u_{\nu}\right] .
\end{aligned}
$$

Here the scalar product with respect to the flat metric $\eta$, i.e., ". $\eta$," is simply denoted as "." to ease the notation.

In the following, we will work, as is standard in EOB theory, in the center-of-mass (c.m.) frame of the binary system, i.e. we will take for $U^{\text {as }}$ the following unit timelike, asymptotic four-vector

$$
U^{\text {as }}=\left[\frac{p_{1}+p_{2}}{\mathcal{E}_{\text {real }}}\right]^{+}=\left[\frac{p_{1}+p_{2}}{\mathcal{E}_{\text {real }}}\right]^{-},
$$

where, in the conservative dynamics we are considering, the asymptotic values at $\pm \infty$ of the total four-momenta $\left(p_{1}+p_{2}\right)^{ \pm}$coincide. Here, $\mathcal{E}_{\text {real }}$ denotes the total energy of the binary system (including the rest-mass energy) in the c.m. frame, which is precisely defined as being the (Minkowski) norm of the asymptotic value of $p_{1}+p_{2}$ :

$$
s=\mathcal{E}_{\text {real }}^{2}=-\left(p_{1}^{+}+p_{2}^{+}\right)^{2}=-\left(p_{1}^{-}+p_{2}^{-}\right)^{2} .
$$

In Eq. (2.17) we used the traditional notation $s$ for the first Mandelstam variable.

By combining Eqs. (2.13)-(2.14) above, we obtain the linear map between the two asymptotic values (at $\pm \infty$ ) of the spatial spin vector of the first particle, namely

$$
S_{1}^{+}=R_{1} S_{1}^{-},
$$

where

$$
\begin{aligned}
R_{1} & \equiv B_{\eta}\left(u_{1}^{+} \rightarrow U^{\text {as }}\right) \Lambda_{1}\left[B_{\eta}\left(u_{1}^{-} \rightarrow U^{\text {as }}\right)\right]^{-1} \\
& =B_{\eta}\left(u_{1}^{+} \rightarrow U^{\text {as }}\right) \Lambda_{1} B_{\eta}\left(U^{\text {as }} \rightarrow u_{1}^{-}\right)
\end{aligned}
$$


with an analogous result for $R_{2}$.

The linear operator $R_{1}$ is easily seen to leave $U^{\text {as }}$ invariant: $R_{1} U^{\text {as }}=U^{\text {as }}$. In addition, as all the linear maps involved in Eq. (2.19) preserve the (Minkowski) length, and as $R_{1}$ transforms $S_{1}^{-}$into $S_{1}^{+}$(both spin vectors living in the three-space orthogonal to $U^{\text {as }}$ ), we conclude that the linear map $R_{1}$, which we shall call the spin holonomy of $\mathcal{L}_{1}$, is an $S O(3)$ rotation acting within the three-space orthogonal to $U^{\text {as }}$ (in the asymptotic Minkowski space).

We can express the spin holonomy, Eqs. (2.19), entirely in terms of the incoming asymptotic values by replacing $u_{1}^{+}$by $\Lambda_{1} u_{1}^{-}$, so that

$$
R_{1}=B_{\eta}\left(\Lambda_{1} u_{1}^{-} \rightarrow U^{\text {as }}\right) \Lambda_{1}\left[B_{\eta}\left(u_{1}^{-} \rightarrow U^{\text {as }}\right)\right]^{-1} .
$$

\section{EVALUATION OF THE SPIN HOLONOMY}

In order to proceed with the evaluation of the spin holonomy $R_{1}$, it is convenient to fix a coordinate system as well as an explicit representation of the asymptotic vectors $u_{1}$ and $u_{2}$ at past infinity: $u_{1}^{-}$and $u_{2}^{-}$. We assume that the scattering process is confined to the $x-y$ plane of a Lorentzian coordinate system $x^{\alpha}=(t, x, y, z)$ and that, with respect to this coordinate system, the particle 1 at past infinity is at rest, while the particle 2 moves along the negative $y$ direction (so that the angular momentum of the system is aligned with the positive $z$ axis). [In the following, we will generally use greek indices from the beginning of the alphabet to denote indices pertaining to the special coordinate system $x^{\alpha}$ attached to the incoming state of particle 1.] Denoting the corresponding coordinate frame $\partial / \partial x^{\alpha}$ as $e_{\alpha}=\left\{e_{t}, e_{x}, e_{y}, e_{z}\right\}$ the two incoming four-velocities read

$$
u_{1}^{-}=e_{t}, \quad u_{2}^{-}=\gamma e_{t}-\sqrt{\gamma^{2}-1} e_{y} .
$$

Here, $\gamma$ denotes the relative Lorentz $\gamma$ factor between the incoming particles, i.e.

$$
\gamma \equiv-u_{1}^{-} \cdot u_{2}^{-} .
$$

It will play an important role in all our computations.

The four-velocity, $U^{\text {as }}$, of the c.m. frame then reads

$$
U^{\text {as }}=\frac{m_{1}}{\mathcal{E}_{\text {real }}} u_{1}^{-}+\frac{m_{2}}{\mathcal{E}_{\text {real }}} u_{2}^{-}=\cosh \alpha e_{t}-\sinh \alpha e_{y},
$$

where we have introduced the rapidity parameter $\alpha$ of the boost between the incoming rest frame of particle 1 and the c.m. frame:

$$
\sinh \alpha=\frac{m_{2} \sqrt{\gamma^{2}-1}}{\mathcal{E}_{\text {real }}}, \quad \cosh \alpha=\frac{m_{1}+m_{2} \gamma}{\mathcal{E}_{\text {real }}} .
$$

As stated above, in the scattering process $U^{\text {as }}=U^{- \text {as }}=$ $U^{+ \text {as }}$ is conserved. Equivalently the total linear fourmomentum is conserved,

$$
P_{\text {tot }}=p_{1}^{-}+p_{2}^{-}=p_{1}^{+}+p_{2}^{+} \text {. }
$$

Let us also introduce a coordinate frame $e_{\hat{\alpha}}=$ $\left\{e_{\hat{t}}, e_{\hat{x}}, e_{\hat{y}}, e_{\hat{z}}\right\}$ attached to the center-of-mass frame, with basis vectors

$$
\begin{aligned}
& e_{\hat{t}}=U^{\mathrm{as}}, \quad e_{\hat{x}}=e_{x}, \\
& e_{\hat{y}}=-\sinh \alpha e_{t}+\cosh \alpha e_{y}, \quad e_{\hat{z}}=e_{z} .
\end{aligned}
$$

In this c.m. frame, only the direction of the spatial linear momentum of each particle is changed from $e_{\hat{y}}^{-}=e_{\hat{y}}$ to, say, $e_{\hat{y}}^{+}$. We can then write

$$
p_{1}^{-}=\mathcal{E}_{1} e_{\hat{t}}+p e_{\hat{y}}^{-}, \quad p_{1}^{+}=\mathcal{E}_{1} e_{\hat{t}}+p e_{\hat{y}}^{+}
$$

where $p$ denotes the magnitude of the three-momentum in the c.m. frame (which is, by definition, common to both particles)

$$
\begin{aligned}
\mathcal{E}_{1} & =\sqrt{m_{1}^{2}+p^{2}}=m_{1} \cosh \alpha, \\
\mathcal{E}_{1}+\mathcal{E}_{2} & =\mathcal{E}_{\text {real }}, \quad p=m_{1} \sinh \alpha .
\end{aligned}
$$

We recall that $\mathcal{E}_{1}$ denotes the c.m. energy of the first particle. The change in the direction of the c.m. threemomentum is measured by the c.m. scattering angle $\chi$. Namely, $\chi$ is the angle of rotation between $e_{\hat{y}}^{-}$and $e_{\hat{y}}^{+}$, so that

$$
e_{\hat{y}}^{+} \cdot e_{\hat{y}}^{-}=\cos \chi .
$$

In addition, $\chi$ enters the second Mandelstam variable $t=-\left(p_{1}^{+}-p_{1}^{-}\right)^{2}$ (measuring the invariant momentum transfer),

$$
\sqrt{-t}=2 p \sin \frac{\chi}{2}
$$

Using the definitions above, it is easily checked that

$$
\begin{aligned}
u_{1}^{+} & =\cosh \alpha\left[\cosh \alpha e_{t}-\sinh \alpha e_{y}\right] \\
& +\sinh \alpha\left[\sin \chi e_{x}+\cos \chi\left(-\sinh \alpha e_{t}+\cosh \alpha e_{y}\right)\right],
\end{aligned}
$$

so that the coordinate components $\Lambda_{(1)^{0}}^{\alpha}$ of $\Lambda_{1}$ in our special (particle-1-related) coordinate system, which must satisfy

$$
u_{1}^{+\alpha}=\Lambda_{(1) \beta}^{\alpha} u_{1}^{-\beta}=\Lambda_{(1) 0}^{\alpha},
$$

are explicitly given by

$$
\begin{aligned}
& \Lambda_{(1)^{0}}^{0}=1+\sinh ^{2} \alpha(1-\cos \chi) \\
& \Lambda_{(1)^{0}}^{1}=\sinh \alpha \sin \chi \\
& \Lambda_{(1)^{0}}^{2}=-\sinh \alpha \cosh \alpha(1-\cos \chi) \\
& \Lambda_{(1)^{0}}^{3}=0 .
\end{aligned}
$$

We can then use Eq. (2.20), written in its inverse form, i.e.,

$$
\Lambda_{1}=\left[B_{\eta}\left(\Lambda_{1} u_{1}^{-} \rightarrow U^{\mathrm{as}}\right)\right]^{-1} R_{1} B_{\eta}\left(u_{1}^{-} \rightarrow U^{\mathrm{as}}\right),
$$


to evaluate the remaining components of the matrix $\Lambda_{1}$. Indeed, we know that the components of the rotation matrix $R_{1}$ in the center-of-mass frame (indicated with hatted greek indices) read simply

$$
R_{1}{ }_{\hat{\beta}}^{\hat{\beta}}=\left(\begin{array}{cccc}
1 & 0 & 0 & 0 \\
0 & \cos \theta & \sin \theta & 0 \\
0 & -\sin \theta & \cos \theta & 0 \\
0 & 0 & 0 & 1
\end{array}\right)
$$

Translating this back to our special coordinate frame $e_{\alpha}$ one finds

$$
R_{1}{ }^{\alpha}{ }_{\beta}=\left(\begin{array}{cccc}
\cos \theta+\left(\cosh ^{2} \alpha-1\right)(1-\cos \theta) & \sin \theta \sinh \alpha & \cosh \alpha \sinh \alpha(1-\cos \theta) & 0 \\
\sin \theta \sinh \alpha & \cos \theta & \sin \theta \cosh \alpha & 0 \\
-\cosh \alpha \sinh \alpha(1-\cos \theta) & -\sin \theta \cosh \alpha & -\cosh ^{2} \alpha(1-\cos \theta)+1 & 0 \\
0 & 0 & 0 & 1
\end{array}\right)
$$

Similarly, using for instance Eq. (2.15), one can determine the coordinate components of the boost matrices $B_{\eta}\left(u_{1}^{-} \rightarrow\right.$ $\left.U^{\text {as }}\right)$ and $B_{\eta}\left(U^{\text {as }} \rightarrow u_{1}^{+}\right)$, namely

$$
B_{\eta}\left(u_{1}^{-} \rightarrow U^{\text {as }}\right)^{\alpha}{ }_{\beta}=\left(\begin{array}{cccc}
\cosh \alpha & 0 & -\sinh \alpha & 0 \\
0 & 1 & 0 & 0 \\
-\sinh \alpha & 0 & \cosh \alpha & 0 \\
0 & 0 & 0 & 1
\end{array}\right), \quad B_{\eta}\left(U^{\text {as }} \rightarrow u_{1}^{-}\right)^{\alpha}{ }_{\beta}=\left(\begin{array}{ccccc}
\cosh \alpha & 0 & \sinh \alpha & 0 \\
0 & 1 & 0 & 0 \\
\sinh \alpha & 0 & \cosh \alpha & 0 \\
0 & 0 & 0 & 1
\end{array}\right)
$$

and,

$$
B_{\eta}\left(U^{\text {as }} \rightarrow u_{1}^{+}\right)^{\alpha}{ }_{\beta}=\left(\begin{array}{cccc}
C^{2} s^{2}(C-1)+\left(1-s^{2}\right) C+s^{2} & -S s(C c+C-c) & S\left(s^{2} C^{2}-s^{2} C-c\right) & 0 \\
S s(C c-C-c) & s^{2} C+1-s^{2} & S^{2} s \frac{(-1+C c-C)}{(1+C)} & 0 \\
-S\left(s^{2} C^{2}-s^{2} C+c\right) & S^{2} s \frac{(1+C c+C)}{(1+C)} & -C\left(s^{2} C^{2}-s^{2} C-1\right) & 0 \\
0 & 0 & 0 & 1
\end{array}\right)
$$

where we shortened the notation as

$$
[s, c, S, C]=[\sin \chi, \cos \chi, \sinh \alpha, \cosh \alpha]
$$

Using Eq. (3.14), we can then determine the remaining nonvanishing components of the matrix $\Lambda$ (in our special coordinate frame) as being given by

$$
\begin{array}{rl}
\Lambda_{(1)^{0}}^{0}= & -c C^{2}+c+C^{2} \\
\Lambda_{(1)^{1}=}^{0} & -S s(-c-C+C c) \cos \theta \\
& +S(-1+c)(C c-c-1) \sin \theta \\
\Lambda_{(1)^{2}=}^{0} & -S(-1+c)(C c-c-1) \cos \theta \\
& -S s(-c-C+C c) \sin \theta \\
\Lambda_{(1)^{0}=}^{1} & S s
\end{array}
$$

$$
\begin{aligned}
\Lambda_{(1) 1}^{1}= & \left(-C c^{2}+C+c^{2}\right) \cos \theta \\
& -c s(C-1) \sin \theta \\
\Lambda_{(1)^{2}}^{1}= & c s(C-1) \cos \theta \\
& +\left(-C c^{2}+C+c^{2}\right) \sin \theta \\
\Lambda_{(1) 0}^{2}= & C(-1+c) S \\
\Lambda_{(1) 1}^{2}= & (C-1) s(-C+C c-1) \cos \theta \\
& +\left(-c-C+c C^{2}+C c^{2}-C^{2} c^{2}\right) \sin \theta \\
\Lambda_{(1)^{2}}^{2}= & \left(C^{2} c^{2}-c C^{2}-C c^{2}+c+C\right) \cos \theta \\
& +(C-1) s(-C+C c-1) \sin \theta .
\end{aligned}
$$

The most useful component for our computations below will be $\Lambda_{(1)^{2}}^{1}=\Lambda_{(1) y}^{x}$. Actually, as we will evaluate this component to the $2 \mathrm{PM}$ accuracy only, we can use a simplified, 2PM-accurate, form of this expression. Indeed, it is easily checked that if one knows $\chi$ and $\theta$ to their 2PM-expanded accuracy: $\chi=\chi_{\exp }=$ $G \chi_{1}+G^{2} \chi_{2}+O\left(G^{3}\right)$ and $\theta=\theta_{\exp }=G \theta_{1}+G^{2} \theta_{2}+O\left(G^{3}\right)$, we have the sufficiently accurate simplified expression

$$
\Lambda_{(1)^{2}}^{1}=\theta_{\exp }+(\cosh \alpha-1) \chi_{\exp }+O\left(G^{3}\right) .
$$

The list of all components in expanded form follows be- 
low:

$$
\begin{aligned}
\Lambda_{(1)^{0}}^{0} \approx & 1+\frac{1}{2}\left(\cosh ^{2} \alpha-1\right) \chi_{1}^{2} G^{2} \\
\Lambda_{(1)^{1}}^{0} \approx & \sinh \alpha \chi_{\exp } \\
\Lambda_{(1)^{2}}^{0} \approx & -\sinh \alpha\left[\left(1-\frac{1}{2} \cosh \alpha\right) \chi_{1}^{2}-\theta_{1} \chi_{1}\right] G^{2} \\
\Lambda_{(1)^{0}}^{1} \approx & \sinh \alpha \chi_{\exp } \\
\Lambda_{(1)^{1}}^{1} \approx & 1+\left[(1-\cosh \alpha)\left(\chi_{1} \theta_{1}-\chi_{1}^{2}\right)-\frac{1}{2} \theta_{1}^{2}\right] G^{2} \\
\Lambda_{(1)^{2}}^{1} \approx & \theta_{\exp }-(1-\cosh \alpha) \chi_{\exp } \\
\Lambda_{(1)^{0}}^{2} \approx & -\frac{1}{2} \sinh \alpha \cosh \alpha \chi_{1}^{2} G^{2} \\
\Lambda_{(1)^{1}}^{2} \approx & -\theta_{\exp }+(1-\cosh \alpha) \chi_{\exp } \\
\Lambda_{(1)^{2}}^{2} \approx & 1+\left[-\frac{1}{2}(1+\cosh \alpha) \chi_{1}^{2}\right. \\
& \left.+\chi_{1} \theta_{1}(1-\cosh \alpha)-\frac{1}{2} \chi_{1}^{2}-\frac{1}{2} \theta_{1}^{2}\right] G^{2} .(3.23
\end{aligned}
$$

As we see, the only components giving $2 \mathrm{PM}$ access to the spin rotation angle $\theta=\theta_{\exp }$ are $\Lambda_{(1)^{2}}^{1}$ and $\Lambda_{(1) 1}^{2}$. In the next sections we will show how to compute the $2 \mathrm{PM}$ accurate value of $\Lambda_{(1) 2}^{1}$ (in our special frame). This will allow us to compute the $2 \mathrm{PM}$-accurate value of the spin holonomy rotation angle $\theta$.

\section{THE TWO-BODY METRIC AT THE 2PM ORDER}

As explained above, to compute the spin holonomy one needs to evaluate the connection one-form associated with the metric $g_{\mu \nu}\left(m_{1}, m_{2}\right)$ generated by the two bodies (along the worldline of one of the two bodies, say the body 1$)$. The $2 \mathrm{PM}$-accurate value of $g_{\mu \nu}\left(m_{1}, m_{2}\right)$ has been explicitly derived (and regularized) long ago in Ref. [20]. In any given Lorentzian coordinate system $x^{\mu}$, with associated Minkowski flat metric $\eta_{\mu \nu}=$ $\operatorname{diag}[-1,+1,+1,+1]$, the solution is conveniently written in terms of the "gothic metric" $\mathfrak{g}^{\mu \nu}(x)$ (see Eq. (93) of Ref. [20])

$$
\mathfrak{g}^{\mu \nu}(x)=\sqrt{-g(x)} g^{\mu \nu}(x) \equiv \eta^{\mu \nu}+\mathrm{h}^{\mu \nu}(x) .
$$

Note that $\mathrm{h}^{\mu \nu}(x)$ denotes the gothic perturbation of the metric, which differs (but is one-to-one) related to the usual metric perturbation $h_{\mu \nu}(x)$, defined by writing

$$
g_{\mu \nu}(x)=\eta_{\mu \nu}+h_{\mu \nu}(x) .
$$

The 2PM solution for the metric of the two-body system found in Ref. [20] is explicitly expressed in terms of the four-velocities of the bodies, $u_{1}$ and $u_{2}$, and their masses. The spacetime dependence of $\mathrm{h}^{\mu \nu}(x)$ goes through the evaluation of retarded time effects. In addition, one should consider only the part of the metric (and the connection) which is "regular" in the vicinity of the worldline of one of the two bodies. This regular part involves both conservative and radiation-reaction effects. We will see that radiation-reaction effects do not contribute to $\theta$, so that our computation based on the retarded solution given in Ref. 20] will give the conservative $2 \mathrm{PM}$ value of $\theta$.

Following Ref. [20], one can write

$$
\begin{aligned}
\mathrm{h}^{\mu \nu} & =G m_{1} \mathrm{~h}_{m_{1}}^{\mu \nu}+G m_{2} \mathrm{~h}_{m_{2}}^{\mu \nu} \\
& +G^{2} m_{1}^{2} \mathrm{~h}_{m_{1}^{2}}^{\mu \nu}+G^{2} m_{1} m_{2} \mathrm{~h}_{m_{1} m_{2}}^{\mu \nu}+G^{2} m_{2}^{2} \mathrm{~h}_{m_{2}^{2}}^{\mu \nu} \\
& =\sum_{n} \mathrm{~h}_{n}^{\mu \nu} .
\end{aligned}
$$

Each one of these terms was given in 20] by an explicit expression involving either the simply-retarded four-velocities of the two bodies, or some doubly-retarded quantities (involving two successive retarded propagations), or (for the most nonlinear terms) the explicit result of a complicated integration involving the cubic vertex of Einstein's action ("P-terms"). We follow the notation and conventions of Ref. [20], apart from the fact that, to follow our previous 1PM work, we shall often use labels 1 and 2 to respectively denote the two masses, by contrast to the unprimed and primed notations used in Ref. [20], where the masses were denoted $m$ and $m^{\prime}$, and the corresponding four-velocities $u$ and $u^{\prime}$, etc.

The main steps necessary to perform the spin holonomy computation are listed below. We recall that our computations will finally be done in a special coordinate frame, see Eq. (3.1), linked to the incoming state of the first particle. We will evaluate the specific component $\Lambda_{(1)^{2}}^{1}=\Lambda_{(1) y}^{x}$ of the scattering holonomy in that special frame.

\section{A. 1PM-accurate orbits of the two bodies}

The solution for $u_{1}\left(\tau_{1}\right)$ and $u_{2}\left(\tau_{2}\right)$ parametrized by their (Minkowski) proper times $\tau_{1}$ and $\tau_{2}$ is obtained by solving the geodesic equations of the $2 \mathrm{PM}$ metric. [As it is customary in PM computations, we use flat space normalization of vectors, ". $\eta$ ", and flat space proper time definitions.] The $2 \mathrm{PM}$ equations of motion are explicitly given in Ref. [20] (see Eqs. (111) there). Here, we will only need their solution at the 1PM order of accuracy. Obtaining this solution is straightforward and leads (in an arbitrary frame) to

$$
\begin{aligned}
u_{1}^{\mu}\left(\tau_{1}\right)= & u_{1}^{-\mu}+G m_{2} \frac{\left(1-2 \gamma^{2}\right) S\left(\tau_{1}\right)}{\sqrt{\gamma^{2}-1} D\left(\tau_{1}\right)} e_{x}^{\mu} \\
& -G m_{2} \frac{\gamma\left(2 \gamma^{2}-3\right)}{\sqrt{\gamma^{2}-1} D\left(\tau_{1}\right)} e_{y}^{\mu},
\end{aligned}
$$


and

$$
\begin{aligned}
z^{\mu}\left(\tau_{1}\right)-z_{1}^{\mu}(0) & =u_{1}^{-\mu} \tau_{1}+G m_{2}\left(1-2 \gamma^{2}\right) \frac{\left(S\left(\tau_{1}\right)-1\right)}{\left(\gamma^{2}-1\right)} e_{x}^{\mu} \\
& -G m_{2} \frac{\gamma\left(2 \gamma^{2}-3\right)}{\gamma^{2}-1} \ln \left(S\left(\tau_{1}\right)\right) e_{y}^{\mu} .
\end{aligned}
$$

Here we have introduced the functions $D(\tau)$ and $S(\tau)$ defined by

$$
D(\tau) \equiv \sqrt{b_{0}^{2}+\tau^{2}\left(\gamma^{2}-1\right)}, \quad D(0)=b_{0},
$$

and

$$
S(\tau) \equiv \frac{1}{b_{0}}\left(\tau \sqrt{\gamma^{2}-1}+D(\tau)\right), \quad S(0)=1 .
$$

Note that $S(\tau) S(-\tau)=1$.

Similarly, the unit tangent vector to the worldline of body 2 is given by

$$
\begin{aligned}
u_{2}{ }^{\mu}\left(\tau_{2}\right) & =u_{2}^{-\mu}-G m_{1} \frac{\left(1-2 \gamma^{2}\right) S\left(\tau_{2}\right)}{\sqrt{\gamma^{2}-1} D\left(\tau_{2}\right)} e_{x}^{\mu} \\
& +G m_{1} \frac{\gamma\left(2 \gamma^{2}-3\right)}{\left(\gamma^{2}-1\right) D\left(\tau_{2}\right)} v^{\prime \mu}
\end{aligned}
$$

and the worldline

$$
\begin{aligned}
{z_{2}}^{\mu}\left(\tau_{2}\right)-z_{2}{ }^{\mu}(0) & =u_{2}^{-\mu} \tau_{2}-G m_{1}\left(1-2 \gamma^{2}\right) \frac{\left(S\left(\tau_{2}\right)-1\right)}{\left(\gamma^{2}-1\right)} e_{x}^{\mu} \\
& +G m_{1} \frac{\gamma\left(2 \gamma^{2}-3\right)}{\left(\gamma^{2}-1\right)^{3 / 2}} \ln \left(S\left(\tau_{2}\right)\right) v^{\prime \mu} .
\end{aligned}
$$

Here the four-vector $v^{\prime}$ is defined as (where $P(u)=\eta+$ $u \otimes u$ denotes the projector orthogonal to $u$ )

$$
v^{\prime} \equiv P\left(u_{2}^{-}\right) u_{1}^{-}=-\left(\gamma^{2}-1\right) e_{t}+\gamma \sqrt{\gamma^{2}-1} e_{y} .
$$

It is the $1 \leftrightarrow 2$ analog of the four-vector $v \equiv P\left(u_{1}^{-}\right) u_{2}^{-}=$ $-\sqrt{\gamma^{2}-1} e_{y}$. In the solutions above, we have fixed initial conditions at $\tau=0$ so that

$$
z_{1}(0)=b_{0} e_{x}, \quad z_{2}(0)=0 .
$$

Note that the intrinsic definition of the vector $b_{0} e_{x}$ is such that $z_{1}\left(\tau_{1}\right)-z_{2}\left(\tau_{2}\right)=b_{0} e_{x}$ when $\tau_{1}=\tau_{2}=0$, which corresponds to connecting the two 1PM-accurate worldlines by a bi-podal line orthogonal to both worldlines. The parameter $b_{0}$ (which is a relativistically defined 1PM-accurate closest distance of approach) differs by an $O(G)$ term from the usually defined c.m. impact parameter $b$ (defined by the condition that the c.m. angular momentum $L$ is equal to $L=b p$, where $p$ is the c.m. three-momentum). We derive in Appendix D the following relation between $b$ and $b_{0}$ :

$$
b=b_{0}+G\left(m+m^{\prime}\right) \frac{\left(2 \gamma^{2}-1\right)}{\gamma^{2}-1}+O\left(G^{2}\right) .
$$

This relation will be crucial for relating our computation to EOB theory.

Having in hands such explicit expressions for the (1PM-accurate) worldlines allows one to explicitly compute the needed retarded times, and associated retarded quantities, entering the 2PM metric. Indeed, given a generic spacetime point $x$ one can first define the intersection of the past light cone from $x$ with the first worldline $\mathcal{L}_{1}$. This defines a point $z_{1}^{R}(x)$ on $\mathcal{L}_{1}$, corresponding to a value $\tau_{1}^{R}(x)$ of the proper time. The null condition

$$
\left(x-z_{1}\left(\tau_{1}^{R}\right)\right)^{2}=0,
$$

defines the functional link $\tau_{1}^{R}=\tau_{1}^{R}(x)$ etc. Then, from the point $z_{1}\left(\tau_{1}^{R}\right)$ on $\mathcal{L}_{1}$ one can draw a second past light cone which intersects the worldline $\mathcal{L}_{2}$ of body 2 at the doubly retarded point $z_{2}^{R R}=z_{2}\left(\tau_{2}^{R R}(x)\right)$. One can determine this doubly-retarded point on $\mathcal{L}_{2}$ by using the null condition

$$
\left(z_{1}\left(\tau_{1}^{R}\right)-z_{2}\left(\tau_{2}^{R R}\right)\right)^{2}=0,
$$

thereby obtaining $\tau_{2}^{R R}$ as a function of $\tau_{1}^{R}(x)$ and hence as a function of the spacetime point $x$ itself.

\section{B. Regular terms in the 2PM metric, and its derivative}

Let us henceforth consider the explicit evaluation of 2PM metric (and its derivative) in the special frame where we shall perform our computation of the scattering holonomy $\Lambda_{1}$ (hence our use of greek indices from the beginning of the alphabet). We recall that, in order to compute $\Lambda_{1}$, we must evaluate the regularized value of the metric, and of the Levi-Civita connection, along the first worldline $\mathcal{L}_{1}$. For detailed discussions of regularization within a PM framework see, e.g., Refs [23] and [20].

At first order in $G$, we have the following contributions to the $2 \mathrm{PM}$ metric (4.17):

$$
\begin{aligned}
G m_{1} \mathrm{~h}_{m_{1}}^{\alpha \beta} & =-4 G m_{1}\left(\frac{u_{1}^{\alpha} u_{1}^{\beta}}{r_{1}}\right)_{R} \\
G m_{2} \mathrm{~h}_{m_{2}}^{\alpha \beta} & =-4 G m_{2}\left(\frac{u_{2}^{\alpha} u_{2}^{\beta}}{r_{2}}\right)_{R},
\end{aligned}
$$

where

$$
\begin{aligned}
& r_{1}=-\left(x-z_{1}\left(\tau_{1}\right)\right) \cdot u_{1}\left(\tau_{1}\right) \\
& r_{2}=-\left(x-z_{2}\left(\tau_{2}\right)\right) \cdot u_{2}\left(\tau_{2}\right),
\end{aligned}
$$

with the label $R$ denoting the evaluation at the retarded time $\tau_{1}^{R}(x)$ etc. Going to the worldline of body 1 the term $G m_{1} \mathrm{~h}_{m_{1}}^{\alpha \beta}$ is singular. However, $G m_{1} \mathrm{~h}_{m_{1}}^{\alpha \beta}$ also contains a regular part. Using, e.g., Ref. [23] for the evaluation of this regular part, and of its derivatives, one can explicitly check that the regular part of $G m_{1} \mathrm{~h}_{m_{1}}^{\alpha \beta}$ does not 
contribute to our computation. The second contribution, $G m_{2} \mathrm{~h}_{m_{2}}^{\alpha \beta}$ is purely regular on the worldline $\mathcal{L}_{1}$. We must keep in mind that, in view of the $1 \mathrm{PM}$-accurate definition of the retarded propertime $\tau_{2}(x)$, it will contribute (when PM-expanded) both at order $G$ and at order $G^{2}$.

At the second order in $G$, we have both "square" terms

$$
\begin{aligned}
G^{2} m_{1}^{2} \mathrm{~h}_{m_{1}^{2}}^{\alpha \beta} & =-G^{2} m_{1}^{2}\left(\frac{7 u_{1}^{\alpha} u_{1}^{\beta}+n_{1}^{\alpha} n_{1}^{\beta}}{r_{1}^{2}}\right)_{R} \\
G^{2} m_{2}^{2} \mathrm{~h}_{m_{2}^{2}}^{\alpha \beta} & =-G^{2} m_{2}^{2}\left(\frac{7 u_{2}^{\alpha} u_{2}^{\beta}+n_{2}^{\alpha} n_{2}^{\beta}}{r_{2}^{2}}\right)_{R},
\end{aligned}
$$

where

$$
\begin{aligned}
& n_{1 R}(x)=\frac{P\left(u_{1 R}\right)\left(x-z_{1}^{R}\right)}{r_{1 R}} \\
& n_{2 R}(x)=\frac{P\left(u_{2 R}\right)\left(x-z_{2}^{R}\right)}{r_{2 R}},
\end{aligned}
$$

and "mixed" terms proportional to $G^{2} m_{1} m_{2} \mathrm{~h}_{m_{1} m_{2}}^{\alpha \beta}$. We again find that the singular contribution $G^{2} m_{1}^{2} \mathrm{~h}_{m_{1}^{2}}^{\alpha \beta}$ does not contribute to our calculation.

Finally among the "mixed" terms we distinguish three different contributions:

$$
\begin{aligned}
G^{2} m_{1} m_{2} \mathrm{~h}_{m_{1} m_{2}}^{\alpha \beta}= & G^{2} m_{1} m_{2} \mathrm{~h}_{m_{1} m_{2}}^{1 /(r \rho) \alpha \beta} \\
& +G^{2} m_{1} m_{2} \mathrm{~h}_{m_{1} m_{2}}^{1 /\left(r^{\prime} \rho^{\prime}\right) \alpha \beta} \\
& +G^{2} m_{1} m_{2} \mathrm{~h}_{m_{1} m_{2}}^{P}{ }^{\alpha \beta}
\end{aligned}
$$

with

$$
\begin{aligned}
G^{2} m_{1} m_{2} \mathrm{~h}_{m_{1} m_{2}}^{1 /(r \rho) \alpha \beta} & =-4 G^{2} m_{1} m_{2}\left(1+2 \gamma^{2}\right)\left(\frac{u_{1}^{\alpha} u_{1}^{\beta}}{r_{1} \rho_{1}}\right)_{R} \\
G^{2} m_{1} m_{2} \mathrm{~h}_{m_{1} m_{2}}^{1 /\left(r^{\prime} \rho^{\prime}\right) \alpha \beta} & =-4 G^{2} m_{1} m_{2}\left(1+2 \gamma^{2}\right)\left(\frac{u_{2}^{\alpha} u_{2}^{\beta}}{r_{2} \rho_{2}}\right)_{R}
\end{aligned}
$$

where

$$
\begin{aligned}
& \rho_{1 R}=-\left(z_{1}^{R}-z_{2}^{R R}\right) \cdot u_{2 R} \\
& \rho_{2 R}=-\left(z_{2}^{R}-z_{1}^{R R}\right) \cdot u_{1 R} .
\end{aligned}
$$

Again, of these two terms the first one (involving $r_{1}$ ) is singular and can be discarded in our calculation. Finally the last "P"-term, $G^{2} m_{1} m_{2} \mathrm{~h}_{m_{1} m_{2}}^{P \beta}$ can be written as

$$
\begin{aligned}
G^{2} m_{1} m_{2} \mathrm{~h}_{m_{1} m_{2}}^{\alpha \beta}= & G^{2} m_{1} m_{2}\left[M_{1}^{\alpha \beta} \rho \sigma D^{\rho} P^{\sigma}\right. \\
& +M_{2}^{\alpha \beta} \rho \sigma D^{\prime \rho} P^{\prime \sigma} \\
& +N_{1}^{\alpha \beta} \rho \sigma D^{\prime \sigma} P^{\rho} \\
& \left.+N_{2}^{\alpha \beta} \rho \sigma D^{\sigma} P^{\prime \rho}\right]_{R}
\end{aligned}
$$

where

$$
\begin{aligned}
M_{1}^{\alpha \beta} \rho \sigma= & -16 u_{1}^{\alpha} u_{1}^{\beta} u_{2 \rho} u_{2 \sigma} \\
N_{1}^{\alpha \beta} \rho \sigma= & 8\left(2 u_{1}^{\alpha} u_{2}{ }^{\beta}-\gamma \eta^{\alpha \beta}\right) u_{2 \rho} u_{1 \sigma} \\
& +\left[-16 \gamma u_{1}^{\alpha} u_{2}{ }^{\beta}-2 \eta^{\alpha \beta}\left(2 \gamma^{2}-1\right)\right] \eta_{\rho \sigma} \\
& +16 \gamma u_{2}{ }^{\alpha} u_{1 \sigma} \delta_{\rho}^{\beta}+16 \gamma u_{2}{ }^{\beta} u_{1 \sigma} \delta_{\rho}^{\alpha} \\
& +4\left(2 \gamma^{2}-1\right) \delta_{\rho}^{\alpha} \delta_{\sigma}^{\beta}
\end{aligned}
$$

and where the derivative $D$ and $D^{\prime}$ are "worldline" derivatives, defined in Eq. (29) of Ref. 20]. Essentially, $h^{\mu} D_{\mu}$ (acting on some functional of the two worldlines, and a function of some given spacetime point $x$ ) denotes the geometric operation consisting in infinitesimally translating (as a whole) the first worldline $\mathcal{L}_{1}$ by the vectorial amount $h^{\mu}$. In view of the overall translation invariance of our problem, the combined derivative $\partial_{\mu}+D_{\mu}+D_{\mu}^{\prime}$ yields a vanishing result on any functional entering our problem. We note also that when $D_{\mu}$ acts on a functional defined (with sufficient accuracy) from straight worldlines (as is the case for $P_{\mu}$ and $P_{\mu}^{\prime}$ ), the longitudinal derivatives $u^{\mu} D_{\mu}$ and $u^{\prime \mu} D_{\mu}^{\prime}$ yield vanishing results.

The four-vectors $P_{\mu}$ and $P_{\mu}^{\prime}$ entering the definition of $\mathrm{h}_{m_{1} m_{0}{ }^{\alpha \beta}}$ (which were one of the crucial new results of Ref. [20]) are defined by nontrivial integrals (involving the cubically nonlinear gravitational vertex), and were explicitly computed in Ref. [20] (see Appendix C there). In addition, Appendix $\mathrm{C}$ there also gave all needed explicit formulas for evaluating the regularized values of $P_{\mu}$ and $P_{\mu}^{\prime}$, and their first derivatives, on the worldlines. See Eqs. (C21)-(C42) there.

As an example of the explicit expressions derived from the above results, we list below the nonvanishing components of the "P" part of the metric with respect to our special chosen coordinate system: 


$$
\begin{aligned}
& \mathrm{h}_{m_{1} m_{2}}^{P t}=-16\left(\gamma^{2}-1\right) D^{2} P^{2}+4\left(3-10 \gamma^{2}\right) D^{\prime 2} P^{2}-4\left(1+6 \gamma^{2}\right) D^{\prime 1} P^{1}-16\left(\gamma^{2}-1\right) D^{\prime 2} P^{\prime 2} \\
& \mathrm{~h}_{m_{1} m_{2}}^{P}{ }^{t x}=4 \frac{\left(2 \gamma^{2}+1\right) \sqrt{\gamma^{2}-1}}{\gamma} D^{\prime 2} P^{1}-16 \gamma \sqrt{\gamma^{2}-1} D^{\prime 1} P^{2} \\
& \mathrm{~h}_{m_{1} m_{2}}^{P}{ }^{t y}=16 \gamma \sqrt{\gamma^{2}-1} D^{\prime 1} P^{1}+12 \frac{\sqrt{\gamma^{2}-1}\left(2 \gamma^{2}-1\right)}{\gamma} D^{\prime 2} P^{2}+16 \frac{\left(\gamma^{2}-1\right)^{3 / 2}}{\gamma} D^{\prime 2} P^{\prime 2} \\
& \mathrm{~h}_{m_{1} m_{2}}^{P x}=4\left(2 \gamma^{2}-1\right) D^{\prime 1} P^{1}+4\left(2 \gamma^{2}-3\right) D^{\prime 2} P^{2} \\
& \mathrm{~h}_{m_{1} m_{2}}^{P} x y=4\left(2 \gamma^{2}-1\right) D^{\prime 1} P^{2}-4\left(2 \gamma^{2}-3\right) D^{\prime 2} P^{1} \\
& \mathrm{~h}_{m_{1} m_{2}}^{P} y y=-4\left(2 \gamma^{2}-1\right) D^{\prime 1} P^{1}+4\left(3-2 \gamma^{2}\right) D^{\prime 2} P^{2}-16 \frac{\left(\gamma^{2}-1\right)^{2}}{\gamma^{2}} D^{\prime 2} P^{\prime 2} \\
& \mathrm{~h}_{m_{1} m_{2}}^{P z}=4\left(-2 \gamma^{2}+1\right) D^{\prime 1} P^{1}+4\left(2 \gamma^{2}-3\right) D^{\prime 2} P^{2} \text {. }
\end{aligned}
$$

\section{COMPUTATION OF $\Lambda_{(1) y}^{x}=\Lambda_{(1) 2}^{1}$}

Having discussed the evaluation of the various regular terms entering the $2 \mathrm{PM}$ metric, we can now compute the connection one-form and, thereby (after integration) the specific scattering holonomy component $\Lambda_{(1) y}^{x}=\Lambda_{(1) 2}^{1}$ necessary to obtain the rotation angle of the center-ofmass spin vector after the full scattering process.

The specific component $\Lambda_{(1) 2}^{1}$ we wish to compute can be decomposed as the sum of various contributions. On the one hand, it contains the contributions obtained by inserting the different terms entering the 2PM metric, Eq. (4.17), into the linear piece, $-\int_{-\infty}^{\infty} \omega_{1}$, of the expanded time-ordered exponential (2.11), say

$$
\begin{aligned}
\Lambda_{(1 \operatorname{lin})_{2}}^{1} & =\sum_{n} \Lambda_{(1)}\left(\mathrm{h}_{n}\right)^{1}{ }_{2} \\
& \equiv \sum_{n} \int_{-\infty}^{+\infty} \Gamma\left(\mathrm{h}_{n}\right)^{1}{ }_{2 \alpha} u_{1}^{\alpha} d \tau
\end{aligned}
$$

On the other hand, one must also add (at the order $G^{2}$ at which we are working) the quadratic contribution in the Dyson expansion (2.11), say

$$
\Lambda_{(1 \text { quad })}^{1}=\int_{-\infty}^{\infty} d \tau \omega_{(1) \sigma}^{1}(\tau) \int_{-\infty}^{\tau} d \tau^{\prime} \omega_{(1)}{ }^{\sigma}{ }_{2}\left(\tau^{\prime}\right) .
$$

All the terms in Eq. (5.1) must be $G$-expanded, up to the order $O\left(G^{2}\right)$ included (and regularized, as explained above). The Christoffel symbols written directly in terms of the gothic metric are given in Eqs. (A12)(A14) of Ref. [20]. We can distinguish four regularized contributions to $\Lambda_{(1)^{2}}^{1}: \mathrm{h}_{1}=G m_{2} \mathrm{~h}_{m_{2}}^{\alpha \beta}$ contributes to both first and second order in $G$; while one gets only $O\left(G^{2}\right)$ contributions from $\mathrm{h}_{2}=G^{2} m_{2}^{2} \mathrm{~h}_{m_{2}^{2}}^{1 /\left(r_{2}{ }^{2}\right)_{\alpha \beta}}$, $\mathrm{h}_{3}=G^{2} m_{1} m_{2} \mathrm{~h}_{m_{1} m_{2}}^{1 /\left(r_{2} \rho_{2}\right)} \alpha \beta$ and $\mathrm{h}_{4}=G^{2} m_{1} m_{2} \mathrm{~h}_{m_{1} m_{2}}^{P}{ }^{\alpha \beta}$. Explicitly, using the notation

$$
u_{1}=u_{1}^{-}+\delta u_{1}
$$

with (in our special coordinate system) $u_{1}^{-\alpha}=\delta_{0}^{\alpha}$ and $\delta u_{1}^{\alpha}=\delta u_{1}^{x} \delta_{x}^{\alpha}+\delta u_{1}^{y} \delta_{y}^{\alpha}$, we find

$$
\begin{aligned}
\Gamma(\mathrm{h})^{1}{ }_{2 \alpha} u_{1}^{\alpha}= & \Gamma\left(\mathrm{h}_{1}\right)^{1}{ }_{2 \alpha}\left(u_{1}^{-\alpha}+\delta u_{1}^{\alpha}\right) \\
& +\sum_{n=2}^{4} \Gamma\left(\mathrm{h}_{n}\right)^{1}{ }_{2 \alpha} u_{1}^{-\alpha}
\end{aligned}
$$

with

$$
\begin{aligned}
\Gamma\left(\mathrm{h}_{1}\right)_{2 \alpha}^{1} u_{1}^{\alpha}= & -\frac{1}{2} \partial_{x} \mathrm{~h}_{1}^{t y} \\
& +\frac{1}{4}\left(\partial_{y} \mathrm{~h}_{1}^{y y}-\partial_{y} \mathrm{~h}_{1}^{t t}\right) \delta u_{1}^{x} \\
& +\frac{1}{4}\left(\partial_{x} \mathrm{~h}_{1}^{t t}+\partial_{x} \mathrm{~h}_{1}^{y y}\right) \delta u_{1}^{y} \\
& +\frac{1}{2}\left(\mathrm{~h}_{1}^{y y}-\mathrm{h}_{1}^{t t}\right) \partial_{x} \mathrm{~h}_{1}^{t y} \\
& -\frac{1}{4} \mathrm{~h}_{1}^{t y}\left(\partial_{x} \mathrm{~h}_{1}^{t t}-\partial_{x} \mathrm{~h}_{1}^{y y}\right),
\end{aligned}
$$

and

$$
\sum_{n=2}^{4} \Gamma\left(\mathrm{h}_{n}\right)^{1}{ }_{2 \alpha} u_{1}^{\alpha}=-\frac{1}{2} \sum_{n=2}^{4}\left(\partial_{x} \mathrm{~h}_{n}^{t y}-\partial_{y} \mathrm{~h}_{n}^{t x}\right) .
$$

For example, the second order contributions from $h_{2,3,4}$ arise from

$$
\int_{-\infty}^{\infty} \Gamma\left(\mathrm{h}_{n}\right)^{1}{ }_{2 \alpha} u_{1}^{-\alpha} d \tau_{1}=-\frac{1}{2} \int_{-\infty}^{\infty} d t\left(\partial_{x} \mathrm{~h}_{n}^{t y}-\partial_{y} \mathrm{~h}_{n}^{t x}\right)
$$

They can be computed relatively easily since, having a $O\left(G^{2}\right)$ integrand, they can be integrated along the 0PM approximation of the worldline of body 1, i.e. a straight worldline: $u_{1}=u_{1}^{-}$, with $u_{1}^{-\alpha}=\delta_{0}^{\alpha}$ with the choice of coordinates adopted here. We then find

$$
\begin{aligned}
& \int_{-\infty}^{\infty} \Gamma\left(\mathrm{h}_{2}\right)^{1}{ }_{2 \alpha} u_{1}^{-\alpha} d \tau_{1}=\frac{15}{4} \pi G^{2} m_{2}^{2} \frac{\gamma}{b_{0}^{2}} \\
& \int_{-\infty}^{\infty} \Gamma\left(\mathrm{h}_{3}\right)^{1}{ }_{2 \alpha} u_{1}^{-\alpha} d \tau_{1}=\pi G^{2} m_{1} m_{2} \frac{\gamma}{b_{0}^{2}}\left(1+2 \gamma^{2}\right) \\
& \int_{-\infty}^{\infty} \Gamma\left(\mathrm{h}_{4}\right)^{1}{ }_{2 \alpha} u_{1}^{-\alpha} d \tau_{1}=-3 \pi G^{2} m_{1} m_{2} \frac{\gamma}{b_{0}^{2}}
\end{aligned}
$$


Particular care should be used, however, for the term $\mathrm{h}_{4}=G^{2} m_{1} m_{2} \mathrm{~h}_{m_{1} m_{2}}^{P \beta}$. One convenient way to proceed is the following. One only needs the two metric components

$$
\begin{aligned}
\mathrm{h}_{m_{1} m_{2}}^{P}{ }^{t x}=-4(1 & \left.+2 \gamma^{2}\right) D^{\prime 0} P^{1}-16 \gamma \sqrt{\gamma^{2}-1} D^{\prime 1} P^{2} \\
\mathrm{~h}_{m_{1} m_{2}}^{P}{ }^{t y}= & -12\left(2 \gamma^{2}-1\right) D^{\prime 0} P^{2} \\
& +16 \gamma \sqrt{\gamma^{2}-1}\left(D^{\prime 0} P^{\prime 0}+D^{\prime 2} P^{2}\right) \\
& +16 \gamma \sqrt{\gamma^{2}-1} D_{\alpha}^{\prime} P^{\alpha} \\
\equiv & \tilde{\mathrm{h}}_{m_{1} m_{2}}^{P t y}+16 \gamma \sqrt{\gamma^{2}-1} D_{\alpha}^{\prime} P^{\alpha}
\end{aligned}
$$

to be used in

$$
\Gamma\left(\mathrm{h}_{4}\right)^{1}{ }_{2 \alpha} u_{1}^{-\alpha}=-\frac{1}{2}\left(\partial_{x} \mathrm{~h}_{4}^{t y}-\partial_{y} \mathrm{~h}_{4}^{t x}\right) .
$$

The regular part of the last term in $\mathrm{h}_{m_{1} m_{2}}^{P}{ }^{t y}$ can be rewritten as [20]

$$
\left[D_{\alpha}^{\prime} P^{\alpha}\right]_{\mathrm{reg}}=-\frac{1}{2 r_{2} \rho_{2}} .
$$

Its contribution to $\Lambda_{(1)}{ }_{2}$ is of the same type as that of $\mathrm{h}_{3}$ and can be evaluated in the same way, leading to the contribution

$$
-2 G^{2} m_{1} m_{2} \pi \frac{\gamma}{b_{0}^{2}}
$$

The contribution due to $\mathrm{h}_{m_{1} m_{2}}^{P}{ }^{t x}$ and the remaining term, $\tilde{\mathrm{h}}_{m_{1} m_{2}}^{{ }^{t y}}{ }^{t y}$, in $\mathrm{h}_{m_{1} m_{2}}^{P}{ }^{t y}$,

$$
-\frac{1}{2}\left(\partial_{x} \tilde{\mathrm{h}}_{4}^{t y}-\partial_{y} \mathrm{~h}_{4}^{t x}\right)
$$

leads to the additional term

$$
-G^{2} m_{1} m_{2} \pi \frac{\gamma}{b_{0}^{2}}
$$

This last computation is performed by: 1) exchanging the partial derivatives with the worldline derivatives; 2 ) replacing $P$ and $P^{\prime}$ with their regular parts using Eqs. (C21) and (C38) of Ref. [20],

$$
\begin{array}{ll}
\partial_{x} P_{x}=R^{(1)}{ }_{x x}, & \partial_{y} P_{x}=R^{(1)}{ }_{x y}, \\
\partial_{x} P_{y}=R^{(1)}{ }_{y x}, & \partial_{y} P_{y}=R^{(1)}{ }_{y y},
\end{array}
$$

and

$$
\partial_{x} P_{0}^{\prime}=R^{\prime(1)}{ }_{x}
$$

taking into account that $R^{(1)}{ }_{x y}=R^{(1)}{ }_{y x}$ and

$$
\begin{aligned}
R_{x y}^{(1)}= & -\frac{1}{4 \rho^{2}}\left[-\frac{A_{x} v_{y}}{A}+\left(A+\frac{A_{y} v_{y}}{A}\right) \frac{A_{x} A_{y}}{A^{2}}\right] \\
R_{x x}^{(1)}= & -\frac{1}{4 \rho^{2}}\left[\frac{A_{x}^{2}}{A}+\frac{A_{x}^{2} A_{y} v_{y}}{A^{3}}-A+\frac{A_{y} v_{y}}{A}\right] \\
R_{y y}^{(1)}= & -\frac{1}{4 \rho^{2}}\left[-\frac{A_{y} v_{y}}{A}+\frac{A_{y}^{2}}{A}+\frac{A_{y}^{3} v_{y}}{A^{3}}-A\right] \\
R_{x}^{\prime(1)}= & \frac{1}{2 \rho^{2}}\left[\left(B_{x}-A_{x}\right) \ln A\right. \\
& \left.+\frac{1}{2} A_{x}\left(1+\frac{2 B}{A}-\frac{2}{A^{2}}\right)+\frac{B_{x}}{2}\right] .
\end{aligned}
$$

Here, entered the components of the following two $u_{1^{-}}$ orthogonal vectors

$$
\mathbf{A}=P\left(u_{1}\right)\left(u_{2}+\nu\right), \quad \mathbf{B}=P\left(u_{1}\right)\left(u_{2}-\nu\right)
$$

as well as

$$
\begin{aligned}
\nu & =\frac{P\left(u_{2}\right)\left[z_{1 R}-z_{2 R R}\right]}{\rho}, \\
\rho & =-\left[z_{1 R}-z_{2 R R}\right] \cdot u_{2} .
\end{aligned}
$$

The above quantities then become functions of $\nu^{x}, \nu^{y}$ and $\rho$ and one can use the expressions for the worldline derivatives of the components of $\nu$ and $\rho$, given in Eqs. (C34) and (C36) of Ref. [20]. The result (5.15) then follows straightforwardly.

Besides the subtle (and intricate) $P$-contribution discussed above, another delicate evaluation concerns the contribution due to the (regularized) linearized gothic metric $\mathrm{h}_{1}$, namely

$$
\begin{aligned}
\mathrm{h}_{1} & =G m_{2} \mathrm{~h}_{m_{2}}^{\alpha \beta}=-4 G m_{2}\left(\frac{u_{2}^{\alpha} u_{2}^{\beta}}{r_{2}}\right)_{R} \\
& =-4 G m_{2} \frac{u_{2}\left(\tau_{2 R}(x)\right)^{\alpha} u_{2}\left(\tau_{2 R}(x)\right)^{\beta}}{r_{2}\left(\tau_{2 R}(x)\right)} .
\end{aligned}
$$

Indeed, this evaluation must be treated with $2 \mathrm{PM}$ accuracy, which means taking into account several $O(G)$ fractional modifications beyond the pure $1 \mathrm{PM}$ evaluation performed in Ref. [18].

The 2PM-accurate contribution associated with $\mathrm{h}_{1}$ has several origins. There is a piece coming from the fact that the first worldline $\mathcal{L}_{1}$ is curved at the $O(G)$ level. The corresponding contribution, proportional to $\delta u_{1}$, was already explicitly written out in Eq. (5.5). The last two lines in Eq. (5.5) exhibit an $\mathrm{h}_{1}$-related contribution that is quadratic in $\mathrm{h}_{1}$. In addition, one should take into account $O\left(G^{2}\right)$ corrections to the pure 1PM evaluation of $\Lambda_{(1)^{2}}^{1}\left(\mathrm{~h}_{1}\right)$ coming from the various $O(G)$ fractional corrections to the retardation effects in $u_{2}$ and $r_{2}$, which enter the definition of $h_{1}$. Indeed, one can formally decompose $\tau_{2 R}(x)$ into the sum of an $O\left(G^{0}\right)$ piece (evaluated as if $\mathcal{L}_{2}$ was straight) and an $O\left(G^{1}\right)$ one (taking into account the $O(G)$ curvature of $\left.\mathcal{L}_{2}\right)$, say

$$
\tau_{2 R}(x)=\tau_{2 R}^{(0)}(x)+G \delta \tau_{2 R}^{(1)}(x)+O\left(G^{2}\right) .
$$

We find

$$
\begin{aligned}
\tau_{2 R}^{(0)}(x)= & \gamma t+\sqrt{\gamma^{2}-1} y \\
& -\sqrt{\left[\sqrt{\gamma^{2}-1} t+\gamma y\right]^{2}+x^{2}},
\end{aligned}
$$

where the first-order correction $\delta \tau_{2 R}^{(1)}(x)$ can be straightforwardly computed from the $O(G)$ term in the $G$ expansion of the defining equation $\left(x-z_{2}\left(\tau_{2 R}\right)\right)^{2}=0$. As a consequence of the expansion (5.22), we have corresponding $G^{0}+G^{1}$ expansions for

$$
u_{2}\left(\tau_{2 R}(x)\right)^{\alpha}=u_{2}^{-\alpha}+G \delta u_{2}^{\alpha}(x)+O\left(G^{2}\right),
$$


and

$r_{2}\left(\tau_{2 R}(x)\right)=\sqrt{\left[\sqrt{\gamma^{2}-1} t+\gamma y\right]^{2}+x^{2}}+G \delta r_{2}(x)+O\left(G^{2}\right)$.

Inserting these expansions in powers of $G$ in the original expression of $h_{1}$ Eq. (5.21), yields the following decomposition of $h_{1}$ :

$$
\mathrm{h}_{1}=G \mathrm{~h}_{1}^{0}+G^{2} \mathrm{~h}_{1}^{\delta u_{2}}+G^{2} \mathrm{~h}_{1}^{\delta r_{2}} .
$$

The term $G \mathrm{~h}_{1}^{0}$ in the latter equation is the linearized metric generated by a straight worldline $\mathcal{L}_{2}$. When inserted in the first term in Eq. (5.5), namely

$$
-\frac{1}{2} \partial_{x} \mathrm{~h}_{1}^{t y}
$$

and integrated, it yields the pure $1 \mathrm{PM}$ contribution to $\Lambda_{1}$, namely

$$
\Lambda_{(1)}^{1 \mathrm{PM}_{2}^{1}}=4 G m_{2} \frac{\gamma}{b_{0}}
$$

Several $O\left(G^{2}\right)$ corrections to this result follow from Eq. (5.5). First, the terms proportional to $\delta u_{1}$ in Eq. (5.5) yield

$$
\begin{aligned}
& \int_{-\infty}^{\infty} \Gamma\left(\mathrm{h}_{1}^{0, \operatorname{lin}}\right)^{1}{ }_{2 \alpha} \delta u_{1}^{\alpha} d \tau_{1}= \\
& \quad+G^{2} m_{2}^{2} \frac{\gamma}{b_{0}^{2}}\left[\pi\left(1+2 \gamma^{2}\right)-4 \frac{\left(2 \gamma^{2}-1\right)}{\gamma^{2}-1}\right]
\end{aligned}
$$

We must then add the contribution to $\Lambda_{(1) 2}^{1}$ coming from the terms $G^{2} \mathrm{~h}_{1}^{\delta u_{2}}+G^{2} \mathrm{~h}_{1}^{\delta r_{2}}$ in Eq. (5.26). These terms can be computed as the ones in Eqs. (5.7), and yield

$$
\begin{aligned}
& \int_{-\infty}^{\infty} \Gamma\left(\mathrm{h}_{1}^{\delta u_{2}}\right)^{1}{ }_{2 \alpha} u_{1}^{-\alpha} d \tau_{1}= \\
& -2 \pi G^{2} m_{1} m_{2} \frac{\gamma}{b_{0}^{2}} \frac{\left(\gamma^{2}-2\right)\left(2 \gamma^{2}-1\right)}{\gamma^{2}-1} \\
& \int_{-\infty}^{\infty} \Gamma\left(\mathrm{h}_{1}^{\delta r_{2}}\right)^{1}{ }_{2 \alpha} u_{1}^{-\alpha} d \tau_{1}= \\
& G^{2} m_{1} m_{2} \frac{\gamma}{b_{0}^{2}}\left[\pi\left(2 \gamma^{2}+1\right)-4 \frac{2 \gamma^{2}-1}{\gamma^{2}-1}\right]
\end{aligned}
$$

Then, the last two terms, quadratic-in- $\mathrm{h}_{1}$, in Eq. (5.5), namely

$$
\frac{1}{2}\left(\mathrm{~h}_{1}^{y y}-\mathrm{h}_{1}^{t t}\right) \partial_{x} \mathrm{~h}_{1}^{t y}-\frac{1}{4} \mathrm{~h}_{1}^{t y}\left(\partial_{x} \mathrm{~h}_{1}^{t t}-\partial_{x} \mathrm{~h}_{1}^{y y}\right)
$$

are found to contribute

$$
\int_{-\infty}^{\infty} \Gamma\left(\mathrm{h}_{1}^{0, \text { quad }}\right)_{2 \alpha} u_{1}^{\alpha} d \tau_{1}=-6 G^{2} m_{2}^{2} \pi \frac{\gamma}{b_{0}^{2}} .
$$

The last $O\left(G^{2}\right)$ correction comes from the quadratic contribution in the Dyson expansion, as written in Eq.
(5.2). This term uses only $h_{1}^{0, l i n}$ and is found to yield the following contribution to $\Lambda_{(1) 2}^{1}$ :

$$
-\frac{1}{2} G^{2} m_{2}^{2} \pi \frac{\gamma}{b_{0}^{2}}\left[4+\frac{1}{\gamma^{2}-1}\right] \text {. }
$$

As a check on our results, we have recomputed all the terms deriving from $h_{1}$ in $\Lambda_{(1)^{2}}^{1}$ in a completely different way, namely by using the Fourier space approach outlined in Ref. [17]. We found complete agreement with those obtained above. Some results of the Fourier space approach are summarized in Appendix A.

\section{A. Final result for the holonomy map}

Summing up all the contributions above, and reexpressing the result in terms of the physical impact parameter $b$ instead of $b_{0}$ (using Eq. (4.12)), we get the final $2 \mathrm{PM}$-accurate result

$$
\Lambda_{(1) 2}^{1}=\frac{4 G m_{2} \gamma}{b}+\frac{G^{2} \pi m_{2} \gamma\left(5 \gamma^{2}-3\right)}{\left(\gamma^{2}-1\right) b^{2}}\left(\frac{3}{4} m_{2}+m_{1}\right) .
$$

It will be most useful to rewrite this result in terms of the total orbital c.m. angular momentum

$$
L=b p_{\mathrm{cm}}=b m_{1} \sinh \alpha .
$$

We introduce the rescaled version $j$ of $L$ defined by

$$
L=G m_{1} m_{2} j
$$

and the relation

$$
b=\frac{G\left(m_{1}+m_{2}\right) h j}{\sqrt{\gamma^{2}-1}},
$$

where $h$ denotes

$$
h \equiv \frac{\mathcal{E}_{\text {real }}}{M}=\frac{\mathcal{E}_{1}+\mathcal{E}_{2}}{m_{1}+m_{2}}=\sqrt{1+2 \nu(\gamma-1)} .
$$

This yields (modulo an $O\left(1 / j^{3}\right)$ error term)

$$
\Lambda_{(1) 2}^{1}=\frac{4 \gamma \sqrt{\gamma^{2}-1}}{j h} X_{2}+\frac{\pi \gamma\left(5 \gamma^{2}-3\right)}{j^{2} h^{2}} X_{2}\left(X_{1}+\frac{3}{4} X_{2}\right)
$$

Using then the relation (3.22), i.e.

$$
\theta_{1}=(1-\cosh \alpha) \chi+\Lambda_{(1) 2}^{1}+O\left(G^{3}\right),
$$

(where we have added a label 1 to $\theta$ as a reminder that this is the spin rotation along $\mathcal{L}_{1}$ ), with

$$
\cosh \alpha=\frac{X_{1}}{h}+\frac{\gamma X_{2}}{h},
$$

as well as the known $2 \mathrm{PM}$ expression of the orbital scattering angle $\chi[21]$

$$
\chi=\frac{2\left(2 \gamma^{2}-1\right)}{j \sqrt{\gamma^{2}-1}}+\frac{3}{4} \frac{\pi}{j^{2}} \frac{5 \gamma^{2}-1}{h},
$$


we finally obtain, at the $2 \mathrm{PM}$ level, the angle of rotation $\theta_{1}$ entering the spin holonomy along $\mathcal{L}_{1}$ :

$$
\begin{aligned}
\theta_{1}= & \frac{2 G}{b\left(\gamma^{2}-1\right)}\left[\left(2 \gamma^{2}-1\right)(h-1) m_{1}\right. \\
& \left.+\left[\left(2 \gamma^{2}-1\right) h-\gamma\right] m_{2}\right] \\
& +\frac{G^{2} \pi}{4 b^{2}\left(\gamma^{2}-1\right)}\left[3\left(5 \gamma^{2}-1\right)(h-1) m_{1}^{2}\right. \\
& +\left[3\left(5 \gamma^{2}-1\right)(2 h-1)\right. \\
& \left.+\gamma\left(5 \gamma^{2}-9\right)\right] m_{1} m_{2} \\
& \left.+3\left[h\left(5 \gamma^{2}-1\right)-2 \gamma\right] m_{2}^{2}\right]
\end{aligned}
$$

or

$$
\begin{aligned}
\theta_{1}= & -\frac{2}{h j \sqrt{\gamma^{2}-1}}\left[\gamma X_{2}+\left(2 \gamma^{2}-1\right)\left(X_{1}-h\right)\right] \\
& +\frac{\pi}{4 h^{2} j^{2}}\left[-3\left(5 \gamma^{2}-1\right)\left(X_{1}-h\right)-6 \gamma X_{2}\right. \\
& \left.+\gamma\left(5 \gamma^{2}-3\right) X_{1} X_{2}\right] .
\end{aligned}
$$

Here we have used either the impact parameter $b$ or the dimensionless orbital angular momentum $j$.

\section{EOB COMPUTATION OF THE SPIN-HOLONOMY ROTATION ANGLE $\theta_{1}$}

In order to transcribe the 2PM-accurate value (5.44) of the spin-holonomy rotation angle $\theta_{1}$ into a corresponding 2PM-accurate value of the two gyrogravitomagnetic factors $g_{S}$ and $g_{S *}$ parametrizing the spin-orbit contribution to the effective EOB Hamiltonian, Eq. (1.6), we need to compute, within the framework of EOB theory, the value of $\theta_{1}$. This was done at the 1PM level in Ref. [18]. The basic physical effect underlying this computation is conceptually very simple (and was already discussed in Ref. [18]). Indeed, the EOB-derived Hamiltonian equation of motion for the spin vector $\mathbf{S}_{1}$ of particle 1 , as obtained from Eq. (1.6), is simply

$$
\frac{d \mathbf{S}_{1}}{d T_{\mathrm{eff}}}=\mathbf{\Omega}_{\mathbf{1}} \times \mathbf{S}_{1}
$$

where $T_{\text {eff }}$ is the effective EOB time (i.e. the evolution parameter with respect to the effective Hamiltonian), and where

$$
\mathbf{\Omega}_{\mathbf{1}}=\frac{\partial H_{\mathrm{eff}}}{\partial \mathbf{S}_{1}}
$$

When considering the linear-in-spin effective EOB Hamiltonian Eq. (1.6), the vectorial angular velocity $\boldsymbol{\Omega}_{\mathbf{1}}$ is simply given by

$$
\boldsymbol{\Omega}_{\mathbf{1}}=G \frac{\mathbf{L}}{R^{3}}\left(g_{S}+\frac{m_{2}}{m_{1}} g_{S *}\right) .
$$

Moreover, when working linearly in the spins, the orbital angular momentum vector $\mathbf{L}$ can be considered as being constant. The integrated vectorial angle of rotation of $\mathbf{S}_{1}$ is then given by

$$
\begin{aligned}
\boldsymbol{\theta}_{1}^{\mathrm{EOB}} & =G \int \boldsymbol{\Omega}_{\mathbf{1}} d T_{\mathrm{eff}} \\
& =G \int \frac{\mathbf{L}}{R^{3}}\left(g_{S}+\frac{m_{2}}{m_{1}} g_{S *}\right) d T_{\mathrm{eff}}
\end{aligned}
$$

To get an explicit integral expression for $\boldsymbol{\theta}_{1}$ we need to express $d T_{\text {eff }}$ in terms of the EOB radial variable $R$. Such an expression is simply obtained by writing the Hamiltonian evolution equation for $R$, namely

$$
\frac{d R}{d T_{\mathrm{eff}}}=+\frac{\partial H_{\mathrm{eff}}}{\partial P_{R}}
$$

At linear order in the spins, it is enough to use the orbital part of $H_{\text {eff }}$, i.e. (with $L=P_{\phi}$ )

$$
H_{\mathrm{eff}}^{\mathrm{orb}}=\sqrt{A(R)\left(\mu^{2}+\frac{P_{R}^{2}}{B(R)}+\frac{L^{2}}{R^{2}}+Q\right)} .
$$

In addition, as we work at the 2PM accuracy, and as there is an explicit $G$ factor in the expression of $\boldsymbol{\theta}_{1}$, it is enough to use the $1 \mathrm{PM}$-accurate EOB effective Hamiltonian. It was shown in Ref. [17] that the 1PMaccurate effective EOB Hamiltonian was simply given by the geodesic dynamics of a particle of mass $\mu$ in a (linearized) Schwarzschild metric of mass $M$, i.e. by an Hamiltonian of the form (6.6) with

$$
\begin{aligned}
& A=1-2 \frac{G M}{R}+O\left(G^{2}\right), \\
& B=1+2 \frac{G M}{R}+O\left(G^{2}\right), \quad Q=0 .
\end{aligned}
$$

Using the equation of motion of $R$, Eq. (6.5), to express $d T_{\text {eff }}$ in terms of $d R$ then leads to the following expression for the (vectorial) rotation angle $\boldsymbol{\theta}_{1}$

$$
\boldsymbol{\theta}_{1}^{\mathrm{EOB}}=G \int \frac{\mathbf{L}}{R^{3}}\left(g_{S}+\frac{m_{2}}{m_{1}} g_{S *}\right) \frac{B}{A} E_{\text {eff }} \frac{d R}{P_{R}},
$$

in which $P_{R}$ should be expressed as a function of $R$ by using the energy conservation (for zero spins), namely

$$
E_{\mathrm{eff}}^{2}=A\left(\mu^{2}+\frac{P_{R}^{2}}{B}+\frac{L^{2}}{R^{2}}\right)
$$

so that

$$
P_{R}^{2}=\frac{B}{A}\left(E_{\mathrm{eff}}^{2}-A\left(\mu^{2}+\frac{L^{2}}{R^{2}}\right)\right) .
$$

In the following, we shall consider the (algebraic) scalar magnitude, $\theta_{1}^{\mathrm{EOB}}$, of the spin rotation, such that $\boldsymbol{\theta}_{1}^{\mathrm{EOB}} / \mathbf{L}=\theta_{1}^{\mathrm{EOB}} / L$.

The gyrogravitomagnetic factors $g_{S}$ and $g_{S *}$ entering Eq. (6.8) are not constant factors but are functions on phase-space. In order to be able to explicitly perform 
the integral over $R$ entering the expression (6.8), it is convenient to choose a spin-gauge which simplifies the phase-space dependence of $g_{S}$ and $g_{S *}$. We recall that Ref. 24] emphasized the existence of a rather large gauge freedom in the phase-space dependence of $g_{S}$ and $g_{S *}$, linked to the freedom in local rotations of the frame used to measure the local components of the spin vectors $\mathbf{S}_{1}$ and $\mathbf{S}_{2}$. In our previous 1 PM-level work Ref. [18], we had conveniently used this freedom to express the $G \rightarrow 0$ limit of $g_{S}$ and $g_{S *}$ as functions of only $\mathbf{P}^{2}$, which was conserved at this order. At our present $2 \mathrm{PM}$ order it will be convenient to use a spin-gauge such that

$$
\begin{aligned}
g_{S} & =g_{S}^{1 \mathrm{PM}}\left(H_{\mathrm{eff}}^{\mathrm{orb}}\right)+g_{S}^{2 \mathrm{PM}}\left(H_{\mathrm{eff}}^{\mathrm{orb}}\right) u+O\left(u^{2}\right), \\
g_{S *} & =g_{S *}^{1 \mathrm{PM}}\left(H_{\mathrm{eff}}^{\mathrm{orb}}\right)+g_{S *}^{2 \mathrm{PM}}\left(H_{\mathrm{eff}}^{\mathrm{orb}}\right) u+O\left(u^{2}\right)
\end{aligned}
$$

As we work linearly in the spins, the use of $H_{\mathrm{eff}}^{\mathrm{orb}}$ as argument is equivalent to using the full $H_{\text {eff }}$ as was indicated in Eq. (1.8) of the Introduction. The crucial point is that the value of this argument can be treated as a constant (equal to $E_{\text {eff }}$, see Eq. (6.9)) during the integration involved in Eq. (6.8). In the following, we shall refer to this gauge as the "energy-gauge".

The integral (6.8) can then be explicitly computed to the required $2 \mathrm{PM}$ accuracy by expanding its integrand in powers of $G$, which means expanding it in powers of

$$
u=\frac{G M}{R} .
$$

Let us use the shorthand notation

$$
\begin{aligned}
g_{S S *} & \equiv g_{S}+\frac{m_{2}}{m_{1}} g_{S *} \\
& =g_{S S *}^{1 \mathrm{PM}}+g_{S S *}^{2 \mathrm{PM}} u+g_{S S *}^{3 \mathrm{PM}} u^{2}+O\left(G^{3}\right) .
\end{aligned}
$$

The various $u$ - or $G$-expansions we need are

$$
A=1-2 u+O\left(G^{2}\right) ; \quad B=1+2 u+O\left(G^{2}\right),
$$

so that

$$
\frac{B}{A}=1+4 u+O\left(G^{2}\right)
$$

and

$$
P_{R}= \pm\left(P_{R}^{(0)}+G P_{R}^{(1)}\right)
$$

with

$$
\begin{aligned}
P_{R}^{(0)} & =\sqrt{E_{\mathrm{eff}}^{2}-\mu^{2}-\frac{L^{2}}{R^{2}}}, \\
G P_{R}^{(1)} & =2 u P_{R}^{(0)}+u \frac{\mu^{2}+\frac{L^{2}}{R^{2}}}{P_{R}^{(0)}} .
\end{aligned}
$$

Substituting in Eq. (6.8) we then formally find

$$
\begin{aligned}
\theta_{1}^{\mathrm{EOB}}= & \\
= & 2 G E_{\mathrm{eff}} L \int_{R_{\min }}^{\infty} \frac{g_{S S *}}{R^{3}}(1+4 u) \times \\
& \times\left(1-G \frac{P_{R}^{(1)}}{P_{R}^{(0)}}\right) \frac{d R}{P_{R}^{(0)}} \\
= & 2 G E_{\mathrm{eff}} \int_{R_{\min }}^{\infty} \frac{g_{S S *}}{R^{3}}\left(1+4 u-G \frac{P_{R}^{(1)}}{P_{R}^{(0)}}\right) \frac{L d R}{P_{R}^{(0)}}
\end{aligned}
$$

where the lower limit of the radial integral is

$$
R_{\min }=\frac{L}{\sqrt{E_{\mathrm{eff}}^{2}-\mu^{2}}}
$$

Note that

$$
P_{R}^{(0)}=\sqrt{E_{\mathrm{eff}}^{2}-\mu^{2}} \sqrt{1-\frac{R_{\mathrm{min}}^{2}}{R^{2}}}
$$

so that $P_{R}^{(0)}$ vanishes at the lower limit (which, indeed, corresponds to the turning point of the $O\left(G^{0}\right)$ radial motion).

A more explicit form of the above integral is then

$$
\theta_{1}^{\mathrm{EOB}}=2 G E_{\mathrm{eff}} R_{\min } \int_{R_{\min }}^{\infty} \frac{d R}{R^{3} \sqrt{1-\frac{R_{\min }^{2}}{R^{2}}}}\left[g_{S S *}^{1 \mathrm{PM}}+u g_{S S *}^{2 \mathrm{PM}}+2 u g_{S S *}^{1 \mathrm{PM}}-u g_{S S *}^{1 \mathrm{PM}} \frac{R_{\min }^{2}}{L^{2}} \frac{\mu^{2}+\frac{L^{2}}{R^{2}}}{1-\frac{R_{\min }^{2}}{R^{2}}}\right]
$$

This integral is formally divergent at the lower limit, where the last term in the integrand has a power-law singularity $\propto\left(1-\frac{R_{\min }^{2}}{R^{2}}\right)^{-3 / 2}$. This divergence was absent in the original, unexpanded form of the integral, and was generated by our formal expansion in powers of $G$. However, some years ago, Damour and Schäfer 25] have proven a general result about such singular integrals obtained by formally expanding (in a perturbation parameter) radial integrals involving turning points that are perturbed during the formal expansion. The correct result is simply obtained by taking the Hadamard partie finie $(\mathrm{PF})$ of the formally expanded (singular) radial 
integrals.

It is technically convenient to use the new variable

$$
x=\frac{R_{\min }^{2}}{R^{2}}, \quad-\frac{1}{2} \frac{d x}{R_{\min }^{2}}=\frac{d R}{R^{3}},
$$

in the integral (6.8). We then get

$$
\begin{aligned}
\theta_{1}^{\mathrm{EOB}}= & \frac{G E_{\mathrm{eff}}}{R_{\min }} g_{S S *}^{1 \mathrm{PM}} \mathrm{PF} \int_{0}^{1} \frac{d x}{\sqrt{1-x}} \\
+ & \frac{G^{2} M E_{\mathrm{eff}}}{R_{\mathrm{min}}^{2}}\left[\left(g_{S S *}^{2 \mathrm{PM}}+2 g_{S S *}^{1 \mathrm{PM}}-g_{S S *}^{1 \mathrm{PM}} \frac{\mu^{2}}{E_{\mathrm{eff}}^{2}-\mu^{2}}\right) \times\right. \\
& \left.\mathrm{PF} \int_{0}^{1} \frac{\sqrt{x} d x}{\sqrt{1-x}}\right] \\
& -g_{S S *}^{1 \mathrm{PM}} \frac{E_{\mathrm{eff}}^{2}}{E_{\mathrm{eff}}^{2}-\mu^{2}} \mathrm{PF} \int_{0}^{1} \frac{x^{3 / 2} d x}{(1-x)^{3 / 2}} .
\end{aligned}
$$

This result is actually valid in any spin-gauge, but it is particularly useful in what we have called above the energy-gauge, as defined by Eq. 6.11). Indeed, in this spin-gauge all the coefficients involving $g_{S S *}^{1 \mathrm{PM}}$ and $g_{S S *}^{2 \mathrm{PM}}$ are constant.

The integral can then be easily obtained, besides using the trivial 1PM-level integral,

$$
\int_{0}^{1} \frac{d x}{\sqrt{1-x}}=2
$$

from the general result:

$\mathrm{PF} \int_{0}^{1} \frac{x^{(2 n+1) / 2} d x}{(1-x)^{(2 n+1) / 2}}=(-1)^{n} \frac{(2 n+1) \pi}{2}, n=0,1,2, \ldots$

leading, in particular, to

$$
\begin{gathered}
\mathrm{PF} \int_{0}^{1} \frac{\sqrt{x} d x}{\sqrt{1-x}}=\frac{\pi}{2} \\
\mathrm{PF} \int_{0}^{1} \frac{x^{3 / 2} d x}{(1-x)^{3 / 2}}=-\frac{3 \pi}{2} .
\end{gathered}
$$

The final result of the integration is:

$$
\begin{aligned}
\theta_{1}^{\mathrm{EOB}} & =\frac{2 \nu}{j} \gamma \sqrt{\gamma^{2}-1} g_{S S *}^{1 \mathrm{PM}} \\
& +\frac{\pi}{2} \frac{\nu}{j^{2}} \gamma\left[\left(5 \gamma^{2}-3\right) g_{S S *}^{1 \mathrm{PM}}+\left(\gamma^{2}-1\right) g_{S S *}^{2 \mathrm{PM}}\right]
\end{aligned}
$$

Here, we used, as above, the dimensionless orbital angular momentum $j=\frac{L}{G M \mu}$, and we have also (to prepare the EOB transcription of the $2 \mathrm{PM}$ rotation angle) replaced $E_{\text {eff }}$ by

$$
\gamma=\frac{E_{\text {eff }}}{\mu} \equiv \hat{E}_{\text {eff }}
$$

It is indeed a noticeable result of EOB theory that the map between the (squared) real c.m. energy, $s=\mathcal{E}_{\text {real }}^{2}$, Eq. (2.17), and the effective EOB energy $E_{\text {eff }}$ is given by the formula (proven in Ref. [17] to be valid to all orders in $v / c$ )

$$
\mathcal{E}_{\text {real }}=M \sqrt{1+2 \nu\left(\frac{E_{\text {eff }}}{\mu}-1\right)},
$$

or, equivalently,

$$
\frac{E_{\text {eff }}}{\mu}=\frac{\left(\mathcal{E}_{\text {real }}\right)^{2}-m_{1}^{2}-m_{2}^{2}}{2 m_{1} m_{2}}
$$

Inserting $s=\mathcal{E}_{\text {real }}^{2}=-\left(p_{1}^{-}+p_{2}^{-}\right)^{2}$ in the latter expression, then yields

$$
\frac{E_{\mathrm{eff}}}{\mu}=-\frac{p_{1}^{-} \cdot p_{2}^{-}}{m_{1} m_{2}}=\gamma
$$

\section{EOB TRANSCRIPTION OF THE SPIN-HOLONOMY ROTATION ANGLE $\theta_{1}$}

As discussed in Ref. [18], the basic condition allowing one to transcribe our 2PM-accurate computation in Sec. [V] of the real, two-body spin-rotation angle $\theta_{1}=\theta_{1}^{\text {real }}$ into information about the EOB spin-orbit couplings is simply the identification

$$
\theta_{1}^{\text {real }}\left(\gamma, L^{\text {real }}, m_{1}, m_{2}\right)=\theta_{1}^{\mathrm{EOB}}\left(E_{\mathrm{eff}}, L^{\mathrm{EOB}}, m_{1}, m_{2}\right) .
$$

Crucial to this identification is the knowledge of the connection between the real dynamical variables (notably $\gamma$, and $L^{\text {real }}$ ) appearing as arguments on the left-hand side, and the effective dynamical variables $\left(E_{\text {eff }}, L^{\mathrm{EOB}}\right)$ appearing on the right-hand side. On the one hand, one of the basic principles of EOB theory is that $L^{\text {real }}=L^{\mathrm{EOB}}$, or $j^{\text {real }}=j^{\mathrm{EOB}}$. On the other hand, we have already explained above the link predicted by EOB theory between the real (incoming) Lorentz factor $\gamma$ and the EOB effective energy $E_{\text {eff }}$. See, Eq. 6.31), which was already used in re-expressing our final result for $\theta_{1}^{\mathrm{EOB}}$.

We note also that, in our energy-spin-gauge, $g_{S}^{1 \mathrm{PM}}$, $g_{S *}^{1 \mathrm{PM}}, g_{S}^{2 \mathrm{PM}}$, and $g_{S *}^{2 \mathrm{PM}}$ must all be functions of only $\gamma=\hat{E}_{\text {eff }}$ and of the symmetric mass ratio $\nu$.

As both sides of the basic identification (7.1) are truncated power series in $1 / j$, we get a separate condition at each PM order. This condition was already solved at the $1 \mathrm{PM}$ order in Ref. [18], with the result (recalling the notation $X_{1} \equiv m_{1} / M, X_{2} \equiv m_{2} / M=1-X_{1}$ )

$$
g_{S S *}^{1 \mathrm{PM}}\left(\gamma, X_{1}\right)=g_{S}^{1 \mathrm{PM}}(\gamma, \nu)+\frac{X_{2}}{X_{1}} g_{S *}^{1 \mathrm{PM}}(\gamma, \nu),
$$


with

$$
\begin{aligned}
g_{S}^{1 \mathrm{PM}}(\gamma, \nu) & =\frac{(2 \gamma+1)(2 \gamma+h)-1}{h(h+1) \gamma(\gamma+1)} \\
& =\frac{1}{h(h+1)}\left[4+\frac{h-1}{\gamma+1}+\frac{h-1}{\gamma}\right] \\
g_{S *}^{1 \mathrm{PM}}(\gamma, \nu) & =\frac{2 \gamma+1}{h \gamma(\gamma+1)} \\
& =\frac{1}{h}\left[\frac{1}{\gamma+1}+\frac{1}{\gamma}\right]
\end{aligned}
$$

The $\nu$-dependence of $g_{S}^{1 \mathrm{PM}}$ and $g_{S *}^{1 \mathrm{PM}}$ is entirely contained in the quantity $h$. We recall that $h \equiv \mathcal{E}_{\text {real }} / M$ is the following function of $\gamma=E_{\text {eff }} / \mu$ and $\nu$ :

$$
h=\sqrt{1+2 \nu(\gamma-1)} .
$$

At the 2PM order, the condition (7.1) gives one equation relating the combination

$$
g_{S S *}^{2 \mathrm{PM}}\left(\gamma, X_{1}\right)=g_{S}^{2 \mathrm{PM}}(\gamma, \nu)+\frac{X_{2}}{X_{1}} g_{S *}^{2 \mathrm{PM}}(\gamma, \nu),
$$

to some given function of $\gamma$, and $X_{1}$, say $f\left(\gamma, X_{1}\right)$. One can extract the two independent (but symmetric under the $1 \leftrightarrow 2$ exchange) functions $g_{S}^{2 \mathrm{PM}}(\gamma, \nu)$ and $g_{S *}^{2 \mathrm{PM}}(\gamma, \nu)$ from the single condition

$$
g_{S}^{2 \mathrm{PM}}(\gamma, \nu)+\frac{X_{2}}{X_{1}} g_{S *}^{2 \mathrm{PM}}(\gamma, \nu)=f\left(\gamma, X_{1}\right)
$$

Indeed, the right-hand side of this equation is a dissymetric function of the two masses. By considering suitable symmetric combinations (such as $f\left(\gamma, X_{1}\right)+f\left(\gamma, X_{2}\right)$ and $\left.\left[f\left(\gamma, X_{1}\right)-f\left(\gamma, X_{2}\right)\right] /\left(X_{1}-X_{2}\right)\right)$ one gets two independent equations allowing one to determine the two symmetric functions $g_{S}^{2 \mathrm{PM}}(\gamma, \nu)$ and $g_{S *}^{2 \mathrm{PM}}(\gamma, \nu)$. [We recall that $\left.\nu \equiv X_{1} X_{2} \cdot\right]$

By equating our two 2PM-accurate results (5.44) and 6.27) we finally get the $2 \mathrm{PM}$-accurate values of the two gyrogravitomagnetic ratios

$$
\begin{aligned}
g_{S}^{2 \mathrm{PM}}(\gamma, \nu) & =-\frac{\nu}{\gamma(\gamma+1)^{2} h^{2}(h+1)^{2}}\left[2(2 \gamma+1)\left(5 \gamma^{2}-3\right) h+(\gamma+1)\left(35 \gamma^{3}-15 \gamma^{2}-15 \gamma+3\right)\right] \\
& =\frac{\nu}{h^{2}(h+1)^{2}}\left[-5(7 \gamma+4 h-10)+\frac{8(3 h-4)}{\gamma+1}-\frac{4 h}{(\gamma+1)^{2}}+\frac{3(2 h-1)}{\gamma}\right] \\
g_{S *}^{2 \mathrm{PM}}(\gamma, \nu) & =-\frac{1}{2 \gamma(\gamma+1)^{2} h^{2}(h+1)}\left[\left(5 \gamma^{2}+6 \gamma+3\right)(h+1)+4 \nu(1+2 \gamma)\left(5 \gamma^{2}-3\right)\right] \\
& =\frac{1}{h^{2}(h+1)}\left[-20 \nu+\frac{24 \nu-h-1}{\gamma+1}+\frac{h+1-4 \nu}{(\gamma+1)^{2}}-\frac{3}{2} \frac{h+1-4 \nu}{\gamma}\right] \\
& =\frac{1}{h^{2}(h+1)}\left[-\frac{20 \gamma \nu}{\gamma+1}+(h+1-4 \nu)\left(\frac{1}{(\gamma+1)^{2}}-\frac{1}{\gamma+1}-\frac{3}{2} \frac{1}{\gamma}\right)\right] .
\end{aligned}
$$

These are the two main results of the present work.

\section{COMPARISON WITH PN-EXPANDED RESULTS}

One of the crucial checks on our 2PM-accurate computation of the gyrogravitomagnetic ratios is their comparison with the known sub-sub-leading order PN-expanded values of these ratios. [This check has helped us to better locate the subtle aspects of the $2 \mathrm{PM}$ calculations explained in Sec. [V] above.] In the present section we restore the presence of the velocity of light $c$ in formulas.

We recall that the $\mathrm{PN}$-expansions of $g_{S}$ and $g_{S *}$ were derived at the next-to-leading order (or sub-leading order) in Ref. 24], and at the sub-sub-leading order in Refs. [26] and [27]. These determinations were done in a general spin gauge, i.e. allowing for a large arbitrariness in the rotational state of the local spin frame.

We have determined the values of the spin-gauge parameters corresponding to the energy-spin-gauge we found most useful in our 2PM calculation. To do so, it is useful to replace the dimensionless effective energy $\hat{E}_{\text {eff }}=E_{\text {eff }} /\left(\mu c^{2}\right)$, or rather the dimensionless effective Hamiltonian $\hat{H}_{\text {eff }}=H_{\text {eff }} /\left(\mu c^{2}\right)$, by the phase-space variable $W(\mathbf{Q}, \mathbf{P})$, such that

$$
\hat{H}_{\mathrm{eff}}^{2}(\mathbf{Q}, \mathbf{P}) \equiv 1+\frac{W(\mathbf{Q}, \mathbf{P})}{c^{2}}
$$

In the $\mathrm{PN}$ expansion, one considers that $1 / c \rightarrow 0$, keeping fixed $W$ as well as the rescaled momenta $\mathbf{p} \equiv \mathbf{P} / \mu$ and $p_{r} \equiv P_{R} / \mu$. To sufficient accuracy, we can use the effective Hamiltonian corresponding to an effective 
Schwarzschild metric (with $u=G M / R$ )

$$
\hat{H}_{\mathrm{eff}}=\sqrt{\left(1-2 \frac{u}{c^{2}}\right)\left(1+\left(1-2 \frac{u}{c^{2}}\right) \frac{p_{r}^{2}}{c^{2}}+\frac{p^{2}}{c^{2}}-\frac{p_{r}^{2}}{c^{2}}\right)} .
$$

[At the present, sub-sub-leading order, we do not need $2 \mathrm{PN}-$ level corrections to the effective metric.]

We can then use the previous relations to express $p$ in terms of $W$ and $p_{r}$ :

$$
\begin{aligned}
p & =\sqrt{W+2 u}+\frac{1}{c^{2}} \frac{\left(2 u^{2}+p_{r}^{2} u+W u\right)}{(W+2 u)^{1 / 2}} \\
& +\frac{1}{c^{4}} \frac{u^{2}\left[-p_{r}^{4}-2(W+2 u) p_{r}^{2}+3 W^{2}+12 W u+12 u^{2}\right]}{2(W+2 u)^{3 / 2}} .
\end{aligned}
$$

Substituting $p^{2}$ into the general gauge-dependent expressions of $g_{S}\left(p^{2}, p_{r}^{2}, u\right)$ and $g_{S *}\left(p^{2}, p_{r}^{2}, u\right)$ derived in [26] allows us to express $g_{S}$ and $g_{S *}$ as functions of the three variables $W, p_{r}^{2}$ and $u$. We can then look for values of the spin-gauge parameters that completely remove all $p_{r}^{2}$ terms. We found a unique set of such gauge-parameters, namely (in the notation of [26])

$$
\begin{aligned}
& a=-\frac{3}{2} \nu, \quad b=-\frac{5}{4} \nu, \quad \eta=\frac{9}{16} \nu+\frac{5}{8} \nu^{2}, \\
& \alpha=-\frac{5}{8} \nu-\frac{7}{4} \nu^{2}, \quad \beta=\frac{27}{16} \nu^{2}, \\
& \delta=\frac{7}{8} \nu-\frac{5}{4} \nu^{2}, \quad \gamma=\frac{7}{8} \nu^{2}, \quad \zeta=\frac{3}{2} \nu^{2} .
\end{aligned}
$$

This allows us to compute the sub-sub-leading PNexpanded values of $g_{S}$ and $g_{S *}$ in the energy-gauge, i.e. as functions of $W$ and $u$

$$
\begin{aligned}
g_{S}(W, u)= & +\frac{1}{c^{2}}\left(-\frac{9}{8} \nu W-\frac{7}{4} u \nu\right) \\
& +\frac{1}{c^{4}}\left[\left(-\frac{129}{8} \nu+\frac{5}{4} \nu^{2}\right) u^{2}\right. \\
& +\left(\frac{9}{4} \nu^{2}-\frac{35}{8} \nu\right) W u \\
& \left.+\left(\frac{1}{8} \nu+\frac{7}{8} \nu^{2}\right) W^{2}\right] \\
g_{S *}(W, u)= & \frac{3}{2}+\frac{1}{c^{2}}\left[-\left(\frac{5}{8}+\frac{3}{4} \nu\right) W-\left(\frac{7}{4}+\frac{3}{2} \nu\right) u\right] \\
& +\frac{1}{c^{4}}\left[\left(\frac{7}{16}+\frac{1}{2} \nu+\frac{9}{16} \nu^{2}\right) W^{2}\right. \\
& +\left(\frac{3}{4}-\nu+\frac{15}{8} \nu^{2}\right) u W \\
& \left.+\left(-\frac{3}{4}-\frac{21}{2} \nu+\frac{9}{8} \nu^{2}\right) u^{2}\right] .
\end{aligned}
$$

We can then re-order these PN expansions according to powers of $u=G M / R$, thereby determining the PNexpanded versions of the coefficients of the PM expansion of $g_{S}$ and $g_{S *}$, namely

$$
\begin{aligned}
g_{S} & =g_{S}^{1 \mathrm{PM}}(\hat{W})+g_{S}^{2 \mathrm{PM}}(\hat{W}) \frac{u}{c^{2}}+g_{S}^{3 \mathrm{PM}}(\hat{W}) \frac{u^{2}}{c^{4}} \\
g_{S *} & =g_{S *}^{1 \mathrm{PM}}(\hat{W})+g_{S *}^{2 \mathrm{PM}}(\hat{W}) \frac{u}{c^{2}}+g_{S *}^{3 \mathrm{PM}}(\hat{W}) \frac{u^{2}}{c^{4}}
\end{aligned}
$$

where $\hat{W}=W / c^{2}$ and

$$
\begin{aligned}
& g_{S}^{1 \mathrm{PM}}(\hat{W})= 2-\frac{9}{8} \nu \hat{W}+\left(\frac{1}{8} \nu+\frac{7}{8} \nu^{2}\right) \hat{W}^{2}+O\left(\hat{W}^{3}\right) \\
& g_{S}^{2 \mathrm{PM}}(\hat{W})=-\frac{7}{4} \nu+\left(\frac{9}{4} \nu^{2}-\frac{35}{8} \nu\right) \hat{W}+O\left(\hat{W}^{2}\right) \\
& g_{S}^{3 \mathrm{PM}}(\hat{W})=-\frac{129}{8} \nu+\frac{5}{4} \nu^{2}+O(\hat{W}) \\
& g_{S *}^{1 \mathrm{PM}}(\hat{W})= \frac{3}{2}-\left(\frac{5}{8}+\frac{3}{4} \nu\right) \hat{W} \\
&+\left(\frac{7}{16}+\frac{1}{2} \nu+\frac{9}{16} \nu^{2}\right) \hat{W}^{2}+O\left(\hat{W}^{3}\right) \\
&+O\left(\frac{7}{4}+\frac{3}{2} \nu\right)+\left(\frac{3}{4}-\nu+\frac{15}{8} \nu^{2}\right) \hat{W} \\
& g_{S *}^{2 \mathrm{PM}}(\hat{W})=-\frac{3}{4}-\frac{21}{2} \nu+\frac{9}{8} \nu^{2}+O(\hat{W}) . \\
& g_{S *}^{3 \mathrm{PM}}(\hat{W})=
\end{aligned}
$$

We have checked that the PN expansion (i.e. the expansion in powers of $\hat{W}$ ) of our 1PM-level, and 2PMlevel, results, Eqs. (7.3), (7.7), fully agree with all the corresponding terms in the PN expansions above.

We note that our new results allow one to replace the current, limited-accuracy PN-expanded versions of the four quantitities $g_{S}^{1 \mathrm{PM}}, g_{S *}^{1 \mathrm{PM}}, g_{S}^{2 \mathrm{PM}}, g_{S *}^{2 \mathrm{PM}}$ by four, exactly known functions of $\hat{W}$. On the other hand, the only knowledge we currently have concerning the $3 \mathrm{PM}$ level is embodied in the two numbers

$$
\lim _{W \rightarrow 0} g_{S}^{3 \mathrm{PM}}(W)=-\frac{129}{8} \nu+\frac{5}{4} \nu^{2},
$$

and

$$
\lim _{W \rightarrow 0} g_{S *}^{3 \mathrm{PM}}(W)=-\frac{3}{4}-\frac{21}{2} \nu+\frac{9}{8} \nu^{2}
$$

\section{HIGH-ENERGY (HE) BEHAVIOR, STRONG-FIELD BEHAVIOR AND RESUMMATION}

\section{A. High-energy (HE) behavior}

One of the great interests in replacing PN-expanded results by PM-extended ones is that it allows one to discuss regimes of the gravitational interaction involving high kinetic energies. Examples of the new insights obtained this way have been discussed in Refs. [17], [18] and [16]. In particular, an interesting property of the high-energy 
behavior of the spin-orbit sector has been pointed out in [18]. Namely, the fact that, at the 1PM level, $g_{S}^{1 \mathrm{PM}}(\gamma, \nu)$, and $g_{S *}^{1 \mathrm{PM}}(\gamma, \nu)$ tend to zero at high-energy (HE), in specific ways, that differ for $g_{S}$ and $g_{S *}$ :

$$
g_{S}^{1 \mathrm{PM}}(\gamma, \nu) \sim \frac{1}{\nu \gamma} \text { as } \gamma \rightarrow \infty
$$

and

$$
g_{S *}^{1 \mathrm{PM}}(\gamma, \nu) \sim \frac{1}{\gamma \sqrt{2 \nu \gamma}} \text { as } \gamma \rightarrow \infty .
$$

It is noticeable that these HE limiting behaviors still hold at the $2 \mathrm{PM}$ level, so that it is tempting to conjecture that they hold at all PM levels. More precisely, we find that, in the HE limit $\gamma \rightarrow \infty$, we have

1.

$$
\begin{aligned}
g_{S}(\gamma, u, \nu) & =g_{S}^{1 \mathrm{PM}}(\gamma, \nu)+u g_{S}^{2 \mathrm{PM}}(\gamma, \nu) \\
& \rightarrow 2\left(1-\frac{35}{8} u\right) \frac{1}{\nu \gamma}+O\left(\frac{1}{\gamma^{3 / 2}}\right)
\end{aligned}
$$

2.

$$
\begin{aligned}
g_{S *}(\gamma, u, \nu) & =g_{S *}^{1 \mathrm{PM}}(\gamma, \nu)+u g_{S *}^{2 \mathrm{PM}}(\gamma, \nu) \\
& \rightarrow 2(1-5 u) \frac{1}{\gamma \sqrt{2 \nu \gamma}}+O\left(\frac{1}{\gamma^{2}}\right) .
\end{aligned}
$$

We conjecture that we would have more generally, as $\gamma \rightarrow \infty$

$$
\begin{gathered}
g_{S}(\gamma, u, \nu) \rightarrow f_{S}(u) \frac{1}{\nu \gamma}+O\left(\frac{1}{\gamma^{3 / 2}}\right) \\
g_{S *}(\gamma, u, \nu) \rightarrow f_{S *}(u) \frac{1}{\gamma \sqrt{2 \nu \gamma}}+O\left(\frac{1}{\gamma^{2}}\right) ;
\end{gathered}
$$

with

$$
\begin{aligned}
f_{S}(u) & =2\left(1-\frac{35}{8} u\right)+O\left(u^{2}\right) ; \\
f_{S *}(u) & =2(1-5 u)+O\left(u^{2}\right) .
\end{aligned}
$$

There are several interesting consequences of these HE behaviors. A consequence concerns the HE behavior of the spin-rotation angle $\theta_{1}$. Let us first recall that the study of the HE limit of the EOB Hamiltonian [16] has shown that it could still be parametrized by an effective metric, with some ( $\nu$-independent!) values of the metric functions $A(R)$ and $B(R)$. [Actually, only the conformal structure of this effective HE metric matters in the HE limit. It is convenient to fix the conformal freedom by using a Schwarzschild-like (areal radius) gauge, and we will do so.] Using then the EOB-predicted integral expression for $\theta_{1}$, Eq. (6.8) (with these effective values of $A(R)$ and $B(R)$ ), and inserting in this integral the limiting behaviors (9.3), (9.4), we first notice that the faster decrease of $g_{S *}$ at $\mathrm{HE}$ implies the relative disappearance of the contribution $\propto \frac{m_{2}}{m_{1}} g_{S *}$. This immediately shows that the spin rotation angle $\theta_{1}$ along $\mathcal{L}_{1}$ will be equal to the spin rotation angle $\theta_{2}$ along $\mathcal{L}_{2}$. Taking into account the various factors of $\nu$ entering into the HE limit of Eq. (6.8), one also finds that the common value, say $\theta^{\text {spin }}$ of $\theta_{1}$ and $\theta_{2}$ has a finite value in the $\mathrm{HE}$ limit, which is independent of $\nu$.

Ref. [16] found that the HE limit of the orbital scattering angle is given by

$$
\frac{\pi}{2}+\frac{\chi}{2} \stackrel{\mathrm{HE}}{=} \int_{0}^{u_{\max }(\bar{\alpha})} d u \frac{\sqrt{A(u) B(u)}}{\sqrt{\bar{\alpha}^{2}-u^{2} A(u)}},
$$

where $u_{\max }(\bar{\alpha})$ is the root of radicand closest to zero, and where we have set

$$
\bar{\alpha} \equiv \frac{\hat{E}_{\mathrm{eff}}}{j} \equiv \frac{G M E_{\mathrm{eff}}}{L} .
$$

The quantity $\bar{\alpha}$ is kept fixed in the HE limit where $E_{\text {eff }}$ and $j$ both tend to infinity.

The corresponding HE limit of $\theta^{\text {spin }}$ is then found to be given by the integral

$$
\frac{\theta^{\text {spin }}}{2} \stackrel{\text { HE }}{=} \int_{0}^{u_{\max }(\bar{\alpha})} u d u f_{S}(u) \sqrt{\frac{B(u)}{A(u)}} \frac{1}{\sqrt{\bar{\alpha}^{2}-u^{2} A(u)}}
$$

The first point we wish to emphasize is that the existence of a finite (mass-independent) $\mathrm{HE}$ limit for $\theta^{\text {spin }}$ has directly followed (within the other tenets of EOB theory) from the special ( $\nu$-dependent!) HE asymptotic behavior (9.3). The second point is that the actual value of the limiting spin-rotation angle $\theta^{\text {spin }}$ is directly related to the value of the renormalized $\mathrm{HE}$ gyrogravitomagnetic ratio $f_{S}(u)$. When using the $1 \mathrm{PM}$ value of $f_{S}(u)$, Eq. (9.5), the computation of the integral (9.9) yields

$$
\theta^{\text {spin }} \stackrel{\mathrm{HE}}{=} 4 \bar{\alpha}+\frac{5}{8} \pi \bar{\alpha}^{2}+O\left(\bar{\alpha}^{3}\right) .
$$

This value can also, evidently, be directly obtained from taking the corresponding limit in the expression (5.44) The corresponding HE value of the orbital scattering angle is [16]

$$
\chi \stackrel{\mathrm{HE}}{=} 4 \bar{\alpha}+O\left(\bar{\alpha}^{3}\right) .
$$

It is interesting to note that, though these values are independent of the mass ratio, the corresponding $\mathrm{HE}$ values of $\theta^{\text {spin }}$ and $\chi$ in the test-particle limit (i.e. when taking $\nu \rightarrow 0$ before taking the HE limit) are different from the above results, but agree among themselves.

Let us first recall the value of $g_{S *}$ for a test particle moving in a spherically symmetric metric [28 30]

$$
g_{S *}^{\mathrm{test}}=\frac{r^{2} \nabla \sqrt{A}}{1+\sqrt{K}}+\frac{r(1-\nabla r) \sqrt{A}}{\sqrt{K}}
$$

where $K \equiv \hat{H}_{\text {eff }}^{2} / A$ and $\nabla \equiv B^{-1 / 2} d / d r$. For the Schwarzschild values of $A$ and $B$ one finds

$$
g_{S *}^{\mathrm{test}}=\frac{\sqrt{1-2 u}}{\hat{E}_{\mathrm{eff}}+\sqrt{1-2 u}}+\frac{1-2 u}{u \hat{E}_{\text {eff }}}(1-\sqrt{1-2 u}) .
$$


Contrary to the previous case where it was $g_{S}$ which was dominating in the HE limit, when the test-particle limit is done before considering the HE limit, it is $g_{S *}$ which dominates. One then finds

$$
\frac{\theta^{\text {test }}}{2} \stackrel{\mathrm{HE}}{=} \int_{0}^{u_{\max }(\bar{\alpha})} d u\left(1-\frac{1-3 u}{\sqrt{1-2 u}}\right) \frac{1}{\sqrt{\bar{\alpha}^{2}-u^{2}(1-2 u)}} .
$$

A direct computation shows that

$$
\int_{0}^{u_{\max }(\bar{\alpha})} d u \frac{1-3 u}{\sqrt{1-2 u}} \frac{1}{\sqrt{\bar{\alpha}^{2}-u^{2}(1-2 u)}}=\frac{\pi}{2}
$$

independent of $\bar{\alpha}$, so that

$$
\theta^{\text {test }} \stackrel{\text { HE }}{=} \chi^{\text {test }},
$$

where $\chi^{\text {test }}$ is the test-particle limit of the HE orbital scattering angle, given by:

$$
\frac{\pi}{2}+\frac{\chi^{\text {test }}}{2} \stackrel{\mathrm{HE}}{=} \int_{0}^{u_{\max }(\bar{\alpha})} d u \frac{1}{\sqrt{\bar{\alpha}^{2}-u^{2} A(u)}}
$$

The beginning of the HE expansions of $\theta^{\text {spin }}$ and $\chi$ read (see [16] for more terms in the expansion)

$$
\chi^{\text {test }} \stackrel{\text { HE }}{=} \theta_{\text {spin }}^{\text {test }} \stackrel{\text { HE }}{=} 4 \bar{\alpha}+\frac{15}{4} \pi \bar{\alpha}^{2}+O\left(\bar{\alpha}^{3}\right) .
$$

\section{B. Strong-field behavior and resummation}

It was noticed early on [24] that the PN corrections to the leading PN-order values of $g_{S}$ and $g_{S *}$, namely $g_{S}^{\mathrm{LO}}=2$ and $g_{S *}^{\mathrm{LO}}=\frac{3}{2}$ tended (for the comparable-mass case, $4 \nu \sim 1$ ) to be all negative, and thereby to diminish the values of $g_{S}$ and $g_{S *}$ in the strong-field regime, i.e. when the two bodies get close to each other so that $u=$ $G M /\left(c^{2} R\right)$ becomes of order unity. In the case of $g_{S *}$, its exact test-mass expression (9.13) shows indeed that $g_{S *}$ contains effects that make it tend to zero both at large energies, and at the horizon $R=2 G M / c^{2}$, i.e. $u=\frac{1}{2}$. We wish to emphasize here that the decrease of $g_{S}$ and $g_{S *}$ as $u$ increases is significantly amplified when considering large energies. Indeed, after having factored the overall power-law decreases of $g_{S}$ and $g_{S *}$ with energy, as in Eqs. (9.3) and (9.4), we see that the linear slope of fractional decrease of $g_{S}$ and $g_{S *}$ as $u$ increases becomes so large (namely $\propto 1-\frac{35}{8} u$ and $\propto 1-5 u$ ) that they would formally predict $g_{S}$ and $g_{S *}$ to vanish at, respectively, separations $R=\frac{35}{8} G M / c^{2}=4.375 G M / c^{2}$ and $R=5 G M / c^{2}$, and then to become negative.

To have $g_{S}$ and $g_{S *}$ changing sign as the two bodies get close to each other does not a priori seem to be physically acceptable. On the other hand, the fact that the HE behavior is factorizable (i.e. seems to be the same at each PM order) suggests a simple way of resumming the PM expansions of $g_{S}(\gamma, u)$ and $g_{S *}(\gamma, u)$. The idea is first to factor out the $\gamma$-dependence that exist at $u=0$, and then to inverse-resum the result of this factorization. In other words, we suggest to replace the standard PM expansions

$$
\begin{aligned}
g_{S, S *}(\gamma, \nu, u)= & g_{S, S *}^{1 \mathrm{PM}}(\gamma, \nu)+u g_{S, S *}^{2 \mathrm{PM}}(\gamma, \nu) \\
& +u^{2} g_{S, S *}^{3 \mathrm{PM}}(\gamma, \nu)+O\left(u^{3}\right)
\end{aligned}
$$

by the following resummed expressions

$$
g_{S, S *}(\gamma, \nu, u)=\frac{g_{S, S *}^{1 \mathrm{PM}}(\gamma, \nu)}{1+u \tilde{c}_{S, S *}^{1}(\gamma, \nu)+u^{2} \tilde{c}_{S, S *}^{2}(\gamma, \nu)},
$$

where

$$
\tilde{c}_{S, S *}^{1}(\gamma, \nu)=-\frac{g_{S, S *}^{2 \mathrm{PM}}(\gamma, \nu)}{g_{S, S *}^{1 \mathrm{PM}}(\gamma, \nu)}
$$

and

$$
\tilde{c}_{S, S *}^{2}(\gamma, \nu)=\left(\frac{g_{S, S *}^{2 \mathrm{PM}}(\gamma, \nu)}{g_{S, S *}^{1 \mathrm{PM}}(\gamma, \nu)}\right)^{2}-\frac{g_{S, S *}^{3 \mathrm{PM}}(\gamma, \nu)}{g_{S, S *}^{1 \mathrm{PM}}(\gamma, \nu)} .
$$

The dependence of the coefficients $\tilde{c}_{S, S *}^{1}(\gamma, \nu)$ on $\gamma$ (and the symmetric mass-ratio $\nu$ ) is shown in Fig. 1 . Both quantities are positive, increasing with $\gamma$ (up to an asymptotic value, $\tilde{c}_{S}^{1} \infty=35 / 8$ and $\tilde{c}_{S *}^{1}{ }^{\infty}=5$, respectively) and have a mild dependence on the symmetric mass ratio $\nu$.

Concerning the next coefficients, $\tilde{c}_{S}^{2}(\gamma, \nu), \tilde{c}_{S *}^{2}(\gamma, \nu)$ we only know their low-energy values, i.e. their values for $\gamma=1$. These are easily deduced from the sub-sub-leading results (8.8), (8.9) above and read

$$
\begin{aligned}
\tilde{c}_{S}^{2}(1, \nu) & =\frac{9}{64} \nu^{2}+\frac{129}{16} \nu, \\
\tilde{c}_{S *}^{2}(1, \nu) & =\frac{7}{16} \nu^{2}+\frac{91}{12} \nu+\frac{125}{72} .
\end{aligned}
$$

In view of the above significant increase of $\tilde{c}_{S}^{1}, \tilde{c}_{S *}^{1}$ as $\gamma$ increases, we might also expect a significant variation of $\tilde{c}_{S}^{2}, \tilde{c}_{S *}^{2}$ with $\gamma$. We recommend to define new EOB codes incorporating the spin-gauge versions of $g_{S}$ and $g_{S *}$ employed in this paper, together with the inverseresummed expressions above, and to compare the predictions of such codes to numerical simulations of two spinning black holes to try to determine best-fit values for $\tilde{c}_{S}^{2}$, and $\tilde{c}_{S *}^{2}$ (and/or for higher-order coefficients). It would also be interesting to transcribe the high-PN-order results on $g_{S}$ and $g_{S *}$ obtained (in a different spin-gauge) from Self-Force theory into the energy-gauge used in this paper. As, in the context of coalescing binary black holes, the effective energy, $\gamma$, varies numerically little around 1 , it might be sufficient to approximate the rather complicated functions $\tilde{c}_{S}^{n}, \tilde{c}_{S *}^{n}$ by simplified expressions.

To give an idea of the values of $g_{S}(\gamma, \nu, u)$ and $g_{S *}(\gamma, \nu, u)$ predicted by our new results, and our new gauge, we plot in Fig. 2 the $u$-dependences predicted by our formulas (together with the leading-PN order values of $\tilde{c}_{S}^{2}$ and $\tilde{c}_{S *}^{2}$ ) for a sample of values of $\gamma$ and $\nu$. 


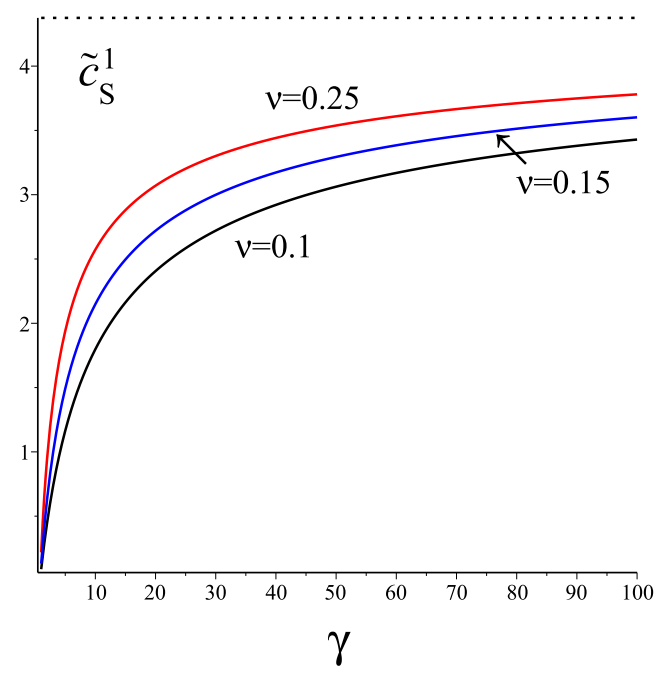

(a)

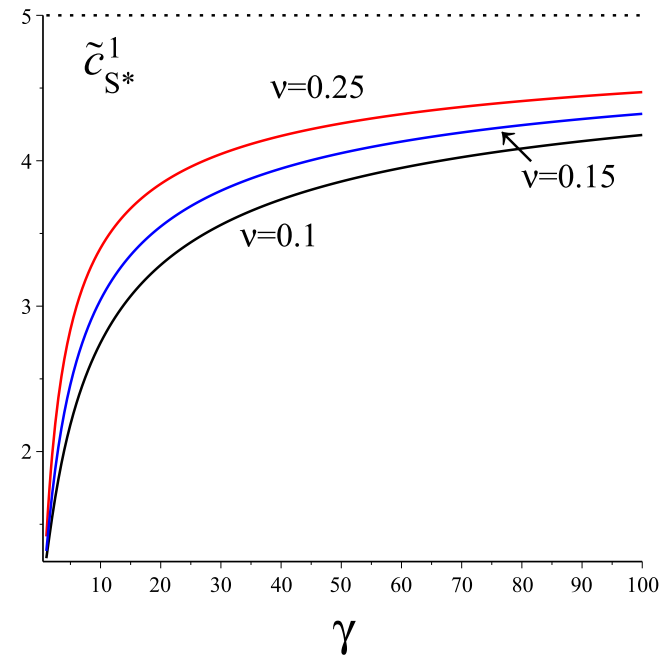

(b)

FIG. 1: Panel (a). The behavior of $\tilde{c}_{S}^{1}$ is shown as a function of $\gamma$ for selected values of $\nu=$ [0.1 (black online), 0.15 (blue online), 0.25 (red online)]. The dotted line corresponds to the asymptotic value $\tilde{c}_{S}^{1} \infty=35 / 8$. Panel (b). The behaviour of $\tilde{c}_{S *}^{1}$ is shown as for $\tilde{c}_{S}^{1}$ with asymptotic value $\tilde{c}_{S *}^{1}{ }^{\infty}=5$.

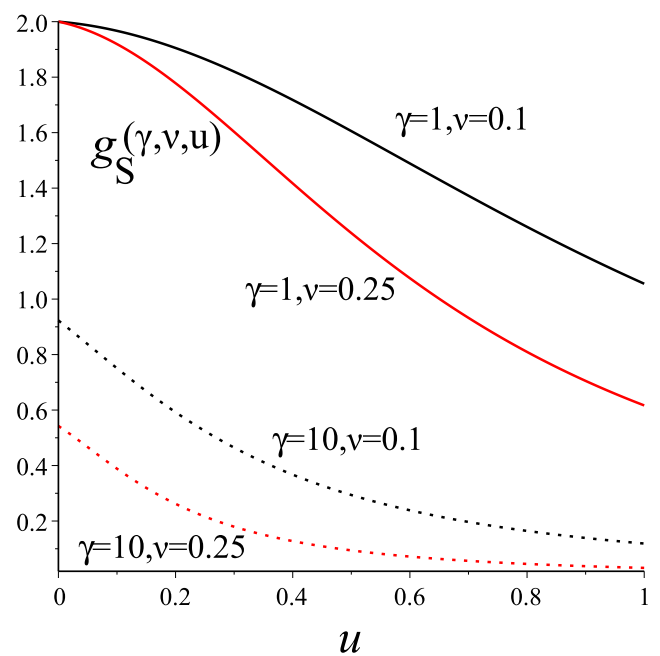

$(a)$

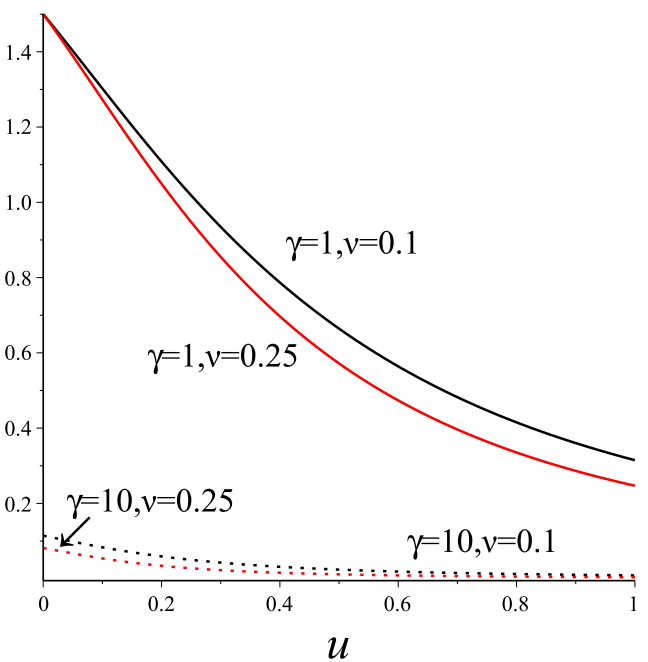

(b)

FIG. 2: Panel (a). The behavior of $g_{S}(\gamma, \nu, u)$ as given in Eq. (9.20) (with known 3PM terms included) is plotted as a function of $u$ for different values of $\gamma=1,10$ and $\nu=0.1$ (black online), 0.25 (red online). Panel (b). $g_{S *}(\gamma, \nu, u)$ is plotted as a function of $u$ for the same parameter choice of panel (a).

We wish also to mention an alternative way of resumming our 2PM results for the gyrogravitomagnetic ratios $g_{S}$ and $g_{S *}$, which is suggested by the (known) structure of the test-particle expression $g_{S *}^{\text {test }}$, Eq. (9.13), for $g_{S *}$ [28 30]. We see on this expression that the numerators and denominators of $g_{S *}(\gamma, u)$ contain linear combinations of $\gamma$ with functions of $u$. When expanding such expressions in powers of $u$ this will generate more com- plicated $\gamma$-dependent denominators, of the type seen in our result for $g_{S, S *}^{2 \mathrm{PM}}(\gamma)$. This suggest to resum $g_{S *}$, at the 2PM accuracy, by an expression of the following type

$$
g_{S *}^{2 \mathrm{PMresum}}=\frac{1}{h}\left[\frac{1+\tilde{a}_{2} u}{\gamma+1+\tilde{a}_{1} u}+\frac{1+\tilde{a}_{3} u}{\gamma}+\tilde{a}_{4} u\right] .
$$

Expanding this expression in powers of $u$ and identifying it (at sub-leading order) with our 2PM-accurate result 
determines the various coefficients entering Eq. (9.25) to be

$$
\tilde{a}_{i}=\frac{a_{i}}{h(h+1)}
$$

with

$$
\begin{aligned}
& a_{1}=-h-1+4 \nu \\
& a_{2}=-h-1-24 \nu \\
& a_{3}=-\frac{3}{2}(h+1)+6 \nu \\
& a_{4}=-20 \nu .
\end{aligned}
$$

A similar resummation of the $2 \mathrm{PM}$-accurate $g_{S}$ is given by

$$
\begin{aligned}
g_{S}^{2 \text { PMresum }}= & \frac{c_{0}+c_{1} u}{h(h+1)+c_{2} \nu u \gamma} \\
& +\frac{1}{\gamma} \frac{-2 \nu h+3 \nu u(2 h-1)}{h^{2}(h+1)^{2}} \\
& +\frac{1}{\gamma+1} \frac{d_{0}+d_{1} u}{\gamma+1+d_{2} u},
\end{aligned}
$$

where

$$
\begin{aligned}
c_{0} & =\frac{4(h+1+\nu)}{h+1} \\
c_{1} & =-\frac{10(2 h-5) \nu}{h(h+1)} \\
c_{2} & =\frac{35(h+1)}{4(h+1+\nu)} \\
d_{0} & =-4 h \nu \\
d_{1} & =8 \nu(-4+3 h) \\
d_{2} & =-1 .
\end{aligned}
$$

One can easily complete these expressions by including the 3PM (i.e., $O\left(u^{2}\right)$ ) known contributions, Eqs. (8.9), (8.9), in the above resummed expressions.

\section{CONCLUDING REMARKS}

Using the notion of spin holonomy (i.e. a suitable projection of the spacetime spin rotation), introduced, at the first post-Minkowskian order, in Ref. [18], we have computed the second-order post-Minkowskian (2PM) corrections to the spin-orbit coupling of a gravitationally interacting, comparable-mass, two-body system. We transcribed our $O\left(G^{2}\right)$-accurate computation of the spin rotation during two-body scattering into the corresponding determination of the gyrogravitomagnetic ratios $g_{S}$ and $g_{S *}$ entering the effective one-body (EOB) Hamiltonian description of linear-in-spin coupling effects. Contrary to the previous, post-Newtonian-based, knowledge of $g_{S}$ and $g_{S *}$, our 2PM-accurate results are exact in $v / c$, and represent a new direction for improving the EOB conservative dynamics of a spinning two-body system.
The computations have been performed in $x$-space by using the explicit form of the 2PM metric generated by two spinless bodies, available since 1981 [20]. Most of our results have been double-checked by Fourier-space ( $k$-space) computations (see Appendix A).

We have indicated ways of resumming our final results so as to use them for defining new versions of spinning EOB codes. In view of future, forthcoming high signalto-noise-ratio gravitational-wave observations, we think that the improved analytical knowledge of the conservative dynamics of a spinning two-body system brought by the present work might play a useful role.

Similarly to the considerations recently made in [16] for the orbital scattering, it would be interesting to explore whether one can extract the classical spin scattering angle from quantum gravitational amplitudes. This would offer a new avenue for further improving the knowledge of spin-orbit couplings in gravitationally interacting systems. Note that our classical 2PM-accurate result corresponds to the quantum one-loop level.

\section{Appendix A: Fourier space computations}

Most of the terms computed above in $x$-space to obtain the spin holonomy matrix component $\Lambda_{(1)}{ }^{1}{ }_{2}$ can be validated by performing analogous calculations in Fourier space. The well-known Fourier-space results

$$
\begin{aligned}
\int \frac{d^{3} k}{(2 \pi)^{3}} \frac{e^{i \vec{k} \cdot \vec{x}}}{k^{2}} & =+\frac{1}{4 \pi|x|} \\
\int \frac{d^{2} K}{(2 \pi)^{2}} \frac{e^{i \vec{K} \cdot \vec{X}}}{K^{2}} & =-\frac{\ln |X|}{2 \pi},
\end{aligned}
$$

where $\vec{k}, \vec{x}$ are vectors of the Euclidean 3 -space and $\vec{K}, \vec{X}$ are vectors in the Euclidean 2-plane, will be often used in the following.

Let us recall that at the linear order in $G$ the gothic metric satisfies

$$
\square \mathrm{h}_{\alpha \beta}=+16 \pi G T_{\alpha \beta} .
$$

The source $T_{\alpha \beta}$ corresponds to the two particles $m_{1}$ and $m_{2}$ and

$$
T_{\alpha \beta}(x)=T_{\alpha \beta}^{\left(m_{1}\right)}(x)+T_{\alpha \beta}^{\left(m_{2}\right)}(x),
$$

where, for example

$$
T_{\alpha \beta}^{\left(m_{2}\right)}(x)=m_{2} \int d \tau^{\prime} u_{2 \alpha}\left(\tau^{\prime}\right) u_{2 \beta}\left(\tau^{\prime}\right) \delta^{(4)}\left(x-z_{2}\left(\tau^{\prime}\right)\right) .
$$

The part of the metric generated by $T^{\left(m_{2}\right)}$ turns out to be given by

$$
\mathrm{h}_{\alpha \beta}(x)=-16 \pi G \int \frac{d^{4} k}{(2 \pi)^{4}} \frac{e^{i k \cdot x}}{k^{2}} T_{\left(m_{2}\right) \alpha \beta}(k),
$$


where

$$
\begin{aligned}
T^{\left(m_{2}\right) \alpha^{\prime} \beta^{\prime}}(k) & =\int d^{4} x e^{-i k \cdot x} T^{\left(m_{2}\right) \alpha^{\prime} \beta^{\prime}}(x) \\
& =m_{2} \int d \tau^{\prime} u_{2}^{\alpha}\left(\tau^{\prime}\right) u_{2}^{\beta}\left(\tau^{\prime}\right) e^{-i k \cdot z_{2}\left(\tau^{\prime}\right)} .
\end{aligned}
$$

Therefore

$$
\begin{aligned}
\mathrm{h}_{\alpha \beta}(x)= & -16 \pi G m_{2} \int \frac{d^{4} k}{(2 \pi)^{4}} \frac{1}{k^{2}} \times \\
& \int d \tau^{\prime} u_{2 \alpha}\left(\tau^{\prime}\right) u_{2 \beta}\left(\tau^{\prime}\right) e^{i k \cdot\left(x-z_{2}\left(\tau^{\prime}\right)\right),}
\end{aligned}
$$

and

$$
\mathrm{h}(x)=16 \pi G m_{2} \int \frac{d^{4} k}{(2 \pi)^{4}} \frac{1}{k^{2}} \int d \tau^{\prime} e^{i k \cdot\left(x-z_{2}\left(\tau^{\prime}\right)\right)},
$$

since $u_{2}\left(\tau^{\prime}\right) \cdot u_{2}\left(\tau^{\prime}\right)=-1+O\left(G^{2}\right)$.

We will compute the following tensors

$$
\begin{aligned}
\mathcal{B}_{\mu \alpha}= & \int \partial_{\mu} \mathrm{h}_{\alpha \beta}(x) d z^{\beta}=\int d \tau\left(\partial_{\mu} \mathrm{h}_{\alpha \beta}(x)\right)_{x=z_{1}(\tau)} u_{1}^{\beta}(\tau) \\
= & -16 \pi G m_{2} \int d \tau u_{1}^{\beta}(\tau) \times \\
& \int \frac{d^{4} k}{(2 \pi)^{4}} \frac{i k_{\mu}}{k^{2}} \int d \tau^{\prime} u_{2 \alpha}\left(\tau^{\prime}\right) u_{2 \beta}\left(\tau^{\prime}\right) e^{i k \cdot\left(z_{1}(\tau)-z_{2}\left(\tau^{\prime}\right)\right)} \\
\mathcal{C}_{\mu}{ }^{\beta}= & \int \partial_{\mu} \mathrm{h}(x) d z^{\beta}=\int d \tau\left(\partial_{\mu} \mathrm{h}\right)_{x=z_{1}(\tau)} u_{1}^{\beta}(\tau) \\
= & 16 \pi G m_{2} \int u_{1}^{\beta}(\tau) d \tau \times \\
& \int \frac{d^{4} k}{(2 \pi)^{4}} \frac{i k_{\mu}}{k^{2}} \int d \tau^{\prime} e^{i k \cdot\left(z_{1}(\tau)-z_{2}\left(\tau^{\prime}\right)\right)} .
\end{aligned}
$$

They enter $\Lambda_{(1)}{ }^{1}$ through the relation

$$
\begin{aligned}
\Lambda_{(1)}{ }^{1}{ }_{2} & =\frac{1}{2}\left(\mathcal{B}_{x y}-\mathcal{B}_{y x}\right)-\frac{1}{4}\left(\mathcal{C}_{x y}-\mathcal{C}_{y x}\right) \\
& =\mathcal{B}_{[x y]}-\frac{1}{2} \mathcal{C}_{[x y]} \\
& \equiv \Lambda_{\mathcal{B}}{ }^{1}{ }_{2}+\Lambda_{\mathcal{C}}{ }^{1}{ }_{2},
\end{aligned}
$$

where one has used the standard notation for antisymmetrization of indices. In fact, from the first-order relation

$$
\begin{aligned}
2 \Gamma^{x}{ }_{y \mu} u_{1}^{\mu}= & \partial_{x} \mathrm{~h}_{y \gamma} u_{1}^{\gamma}-\partial_{y} \mathrm{~h}_{x \gamma} u_{1}^{\gamma} \\
& -\frac{1}{2} u_{1}^{y} \partial_{x} \mathrm{~h}+\frac{1}{2} u_{1}^{x} \partial_{y} \mathrm{~h},
\end{aligned}
$$

one immediately obtains Eq. (A10).

\section{The case of straight lines}

In this limit the worldlines of the two particles read

$$
z_{1}(\tau)=b_{0} \partial_{x}+u_{1}^{-} \tau, \quad z_{2}\left(\tau^{\prime}\right)=u_{2}^{-} \tau^{\prime}
$$

and $b_{0}=b$ coincides with the impact parameter.
1. Computation of $\mathcal{B}_{\mu \alpha}$

Let us start analyzing the case of straight lines

$$
\begin{aligned}
\mathcal{B}_{\mu \alpha}= & +16 \pi G m_{2} \gamma u_{2}^{-} \alpha \int \frac{d^{4} k}{(2 \pi)^{4}} \frac{i k_{\mu}}{k^{2}} e^{i k \cdot b_{0}} \times \\
& \int d \tau e^{i\left(k \cdot u_{1}^{-}\right) \tau} \int d \tau^{\prime} e^{-i\left(k \cdot u_{2}^{-}\right) \tau^{\prime}} \\
= & +16 \pi G m_{2} \gamma u_{2}^{-} \alpha \times \\
& \frac{\partial}{\partial b_{0}^{\mu}} \int \frac{d^{4} k}{(2 \pi)^{2}} \frac{e^{i k \cdot b_{0}}}{k^{2}} \delta\left(k \cdot u_{1}^{-}\right) \delta\left(k \cdot u_{2}^{-}\right) .(
\end{aligned}
$$

Expressing $\delta\left(k \cdot u_{1}^{-}\right) \delta\left(k \cdot u_{2}^{-}\right)$in the usual coordinate system we find

$\delta\left(k \cdot u_{1}^{-}\right) \delta\left(k \cdot u_{2}^{-}\right)=\frac{1}{\sqrt{\gamma^{2}-1}} \delta\left(k^{0}\right) \delta\left(k^{y}\right)$,

and then, recalling that $\frac{\partial b_{0}}{\partial b_{0}^{\mu}}=\frac{b_{0 \mu}}{b_{0}}$, we find

$$
\begin{aligned}
\mathcal{B}_{\mu \alpha} & =16 \pi G m_{2} \frac{\gamma}{\sqrt{\gamma^{2}-1}} u_{2}^{-} \alpha \frac{\partial}{\partial b_{0}^{\mu}} \int \frac{d k^{x} d k^{z}}{(2 \pi)^{2}} \frac{e^{i k \cdot b_{0}}}{k^{2}} \\
& =-16 \pi G m_{2} \frac{\gamma}{\sqrt{\gamma^{2}-1}} u_{2}-\frac{\partial}{\partial b_{0}^{\mu}} \frac{1}{2 \pi} \ln b_{0} \\
& =-8 G m_{2} \frac{\gamma}{\sqrt{\gamma^{2}-1}} \frac{b_{0 \mu}}{b_{0}^{2}} u_{2}^{-} \alpha,
\end{aligned}
$$

implying

$$
\mathcal{B}_{x y}=8 G m_{2} \frac{\gamma}{b_{0}}, \quad \mathcal{B}_{y x}=0 .
$$

2. Computation of $\mathcal{C}_{\mu \alpha}$

In this case we have

$$
\begin{aligned}
\mathcal{C}_{\mu \beta}= & -16 \pi G m_{2} \delta_{\beta}^{0} \int \frac{d^{4} k}{(2 \pi)^{4}} \frac{i k_{\mu}}{k^{2}} e^{i k \cdot b_{0}} \times \\
& \int d \tau e^{i k \cdot u_{1}^{-} \tau} \int d \tau^{\prime} e^{-i k \cdot u_{2}^{-} \tau^{\prime}} \\
= & -16 \pi G m_{2} \delta_{\beta}^{0} \frac{\partial}{\partial b_{0}^{\mu}} \int \frac{d^{4} k}{(2 \pi)^{4}} \\
& \frac{e^{i k \cdot b_{0}}}{k^{2}}(2 \pi)^{2} \delta\left(k \cdot u_{1}^{-}\right) \delta\left(k \cdot u_{2}^{-}\right) \\
= & -16 \pi G m_{2} \delta_{\beta}^{0} \frac{1}{\sqrt{\gamma^{2}-1}} \frac{b_{0}^{\mu}}{b_{0}^{2}},
\end{aligned}
$$

so that $\mathcal{C}_{x y}=\mathcal{C}_{y x}=0$.

The final result for $\Lambda_{(1)^{2}}^{1}$ at the 1PM order coincides with the well-known result

$$
\Lambda_{(1) 2}^{1}=-\frac{1}{2} \mathcal{B}_{x y}=4 G m_{2} \frac{\gamma}{b_{0}}
$$


In this special case (straight lines) one can easily compute $\partial_{\alpha} \mathrm{h}_{\beta \mu}(x)$, namely

$$
\partial_{\alpha} \mathrm{h}_{\beta \mu}(x)=C_{\alpha} u_{-\beta}^{\prime} u_{-\mu}^{\prime}, \quad \partial_{\alpha} \mathrm{h}(x)=-C_{\alpha}
$$

where

$$
\begin{aligned}
C_{\alpha}(x) & =-16 \pi G m_{2} \int \frac{d^{4} k}{(2 \pi)^{4}} \frac{i k_{\alpha}}{k^{2}}(2 \pi) e^{i k \cdot\left(x-z_{2}(0)\right)} \delta\left(k \cdot u_{2}^{-}\right) \\
& =-16 \pi G m_{2} \frac{\partial}{\partial \xi^{\alpha}} \int \frac{d^{4} k}{(2 \pi)^{3}} \frac{e^{i k \cdot \xi}}{k^{2}} \delta\left(k^{0}+\frac{\sqrt{\gamma^{2}-1}}{\gamma} k^{y}\right) \\
& =-16 \pi G m_{2} \frac{\partial}{\partial \xi^{\alpha}} \frac{1}{4 \pi} \frac{1}{|\xi|}=4 G m_{2} \frac{\xi_{\alpha}}{|\xi|^{3}}, \quad \text { (A20) }
\end{aligned}
$$

where

$$
\begin{aligned}
\xi & =P\left(u_{2}^{-}\right) x=x+\left(u_{2}^{-} \cdot x\right) u_{2}^{-} \\
& =x \partial_{x}+\left(\gamma y+\sqrt{\gamma^{2}-1} t\right) e\left(u_{2}^{-}\right)_{2}+z \partial_{z}
\end{aligned}
$$

with

$$
e\left(u_{2}^{-}\right)_{2}=-\sqrt{\gamma^{2}-1} \partial_{t}+\gamma \partial_{y} .
$$

Along the particle $m_{1}$ worldline

$$
\xi \rightarrow b_{0} \partial_{x}+t \sqrt{\gamma^{2}-1} e\left(u_{2}^{-}\right)_{2},
$$

so that

$$
\begin{aligned}
\frac{\xi_{t}}{|\xi|^{3}} & =\frac{\left(\gamma^{2}-1\right) t}{D(t)^{3}} \\
\frac{\xi_{x}}{|\xi|^{3}} & =\frac{b_{0}}{D(t)^{3}} \\
\frac{\xi_{y}}{|\xi|^{3}} & =\frac{\gamma \sqrt{\gamma^{2}-1} t}{D(t)^{3}} .
\end{aligned}
$$

Replacing

$$
\partial_{\alpha} \mathrm{h}_{\beta \mu}(x)=4 G m_{2} \frac{\xi_{\alpha}}{|\xi|^{3}} u_{-\beta}^{\prime} u_{-\mu}^{\prime}=C_{\alpha} u_{-\beta}^{\prime} u_{-\mu}^{\prime},
$$

implying

$$
u_{-}^{\prime} \cdot C=0, \quad u_{-}^{\prime}{ }^{\alpha} \partial_{\alpha} \mathrm{h}_{\beta \mu}(x)=0,
$$

in the connection term (also evaluated along the worldline of the particle $m_{1}$, with $x=u_{1}^{-} \tau+b_{0}$ and $C^{\alpha}=$ $\left.C^{\alpha}(\tau)\right)$, namely

$$
\begin{aligned}
\omega_{1}(\tau)^{\alpha}{ }_{\beta}= & \frac{1}{2}\left(\partial^{\alpha} \mathbf{h}_{\beta 0}-\partial_{\beta} \mathrm{h}^{\alpha}{ }_{0}-\partial_{0} \mathrm{~h}^{\alpha}{ }_{\beta}\right) \\
& +\left.\frac{1}{4}\left(\delta_{0}^{\alpha} \partial_{\beta} \mathrm{h}+\delta_{\beta}^{\alpha} \partial_{0} \mathrm{~h}+\delta_{\beta}^{0} \partial^{\alpha} \mathrm{h}\right)\right|_{x=x(\tau)}(\mathrm{A} 27
\end{aligned}
$$

we find

$$
\begin{aligned}
\omega_{1}(\tau)^{\alpha}{ }_{\beta}= & \left\{\frac { 1 } { 2 } \left[-\gamma\left(C^{\alpha} u_{2}^{-}{ }^{-\alpha} u_{2}^{-\alpha} C_{\beta}\right)\right.\right. \\
& \left.-C_{0} u_{2}^{-\alpha} u_{2}^{-} \beta\right] \\
& \left.-\frac{1}{4}\left[\delta_{0}^{\alpha} C_{\beta}+\delta_{\beta}^{\alpha} C_{0}+\delta_{\beta}^{0} C^{\alpha}\right]\right\} d \tau \\
= & -\frac{\gamma}{2}\left[C \wedge u_{2}^{-}\right]^{\alpha}{ }_{\beta}+\frac{1}{4}\left[C \wedge u_{1}^{-}\right]^{\alpha}{ }_{\beta} \\
& -\frac{1}{2} C_{0}\left(u_{2}^{-\alpha} u_{2}^{-} \beta+\frac{1}{2} \delta_{\beta}^{\alpha}\right) \\
= & {\left[C_{1}(\tau)^{\alpha}{ }_{\beta}+\mathrm{C}_{2}(\tau)^{\alpha}{ }_{\beta}\right] d \tau, }
\end{aligned}
$$

or explicitly

$$
\omega_{1}(\tau)^{\alpha}{ }_{\beta}=\left(\begin{array}{cccc}
\frac{C^{0}\left(1-2 \gamma^{2}\right)}{4} & -\frac{C^{1}\left(1-2 \gamma^{2}\right)}{4} & \frac{C^{2}\left(1-2 \gamma^{2}\right)}{4} & 0 \\
-\frac{C^{1}\left(1-2 \gamma^{2}\right)}{4} & \frac{C^{0}}{4} & \frac{\gamma \sqrt{\gamma^{2}-1} C^{1}}{2} & 0 \\
-\frac{C^{2}\left(1-2 \gamma^{2}\right)}{4}+\gamma \sqrt{\gamma^{2}-1} C^{0} & -\frac{\gamma \sqrt{\gamma^{2}-1} C^{1}}{2} & -\frac{C^{0}\left(1-2 \gamma^{2}\right)}{4} & 0 \\
0 & 0 & 0 & \frac{C^{0}}{4}
\end{array}\right)
$$

We can compute then

$$
\begin{aligned}
\omega_{1}(\tau)^{\alpha}{ }_{\beta} \omega_{1}\left(\tau^{\prime}\right)_{\mu}^{\beta}= & {\left[\mathrm{C}_{1}(\tau)^{\alpha}{ }_{\beta}+\mathrm{C}_{2}(\tau)^{\alpha}{ }_{\beta}\right] \times } \\
& {\left[\mathrm{C}_{1}\left(\tau^{\prime}\right)^{\beta}{ }_{\mu}+\mathrm{C}_{2}\left(\tau^{\prime}\right)^{\beta}{ }_{\mu}\right] d \tau d \tau^{\prime}, }
\end{aligned}
$$

and finally, using the abbreviated notation $C^{\alpha}=C^{\alpha}(\tau)$ and $C^{\prime \alpha}=C^{\prime \alpha}\left(\tau^{\prime}\right)$,

$$
\begin{aligned}
\omega_{1}(\tau)^{x}{ }_{\beta} \omega_{1}\left(\tau^{\prime}\right)_{y}^{\beta} & =\left\{\frac { 2 \gamma ^ { 2 } - 1 } { 8 } \left[\frac{1}{2}\left(2 \gamma^{2}-1\right) C^{2}\right.\right. \\
& \left.+\gamma \sqrt{\gamma^{2}-1} C^{\prime 0}\right] C^{1} \\
& \left.+\frac{\gamma \sqrt{\gamma^{2}-1}}{8} C^{0} C^{\prime 1}\right\} d \tau^{\prime} d \tau(\mathrm{A} 31)
\end{aligned}
$$

Integrating over $\tau^{\prime} \in(-\infty, \tau)$ and then over $\tau \in$ 
$(-\infty,+\infty)$ gives

$$
\int_{-\infty}^{\infty} \int_{-\infty}^{\tau} \omega_{1}(\tau)^{x}{ }_{\beta} \omega_{1}\left(\tau^{\prime}\right)^{\beta}{ }_{y}=-\frac{\pi G^{2} m_{2}^{2}}{2} \frac{\gamma\left(4 \gamma^{2}-3\right)}{b_{0}^{2}\left(\gamma^{2}-1\right)}
$$

which coincides with the previously obtained result.

\section{Appendix B: The general case: computation of $\mathcal{B}_{\mu \alpha}$}

Start from Eq. (A9) and insert the four velocities and worldlines

$$
\begin{aligned}
z_{1}^{\beta}(\tau) & =z_{1}(0)^{\beta}+u_{1}^{-\beta} \tau+\delta z_{1}^{\beta}(\tau), \\
z_{2}^{\beta}\left(\tau^{\prime}\right) & =z_{2}(0)^{\beta}+u_{2}^{-\beta} \tau^{\prime}+\delta z_{2}^{\beta}\left(\tau^{\prime}\right),
\end{aligned}
$$

with $b_{0}^{\beta}=z_{1}(0)^{\beta}$ and $z_{2}(0)^{\beta}$ vanishing, namely

$$
\mathcal{B}_{\mu \alpha}=-16 \pi G m_{2} \int \frac{d^{4} k}{(2 \pi)^{4}} \frac{i k_{\mu}}{k^{2}} e^{i k \cdot b_{0}} F_{\alpha}(k),
$$

where

$$
\begin{aligned}
F^{\alpha}(k) & =\iint d \tau d \tau^{\prime}\left(u_{1}^{-\beta}+\delta u_{1}^{\beta}(\tau)\right)\left(u_{2}^{-} \beta+\delta u_{2 \beta}\left(\tau^{\prime}\right)\right)\left(u_{2}^{-\alpha}+\delta u_{2}{ }^{\alpha}\left(\tau^{\prime}\right)\right) e^{i k \cdot\left(u_{1}^{-} \tau+\delta z_{1}(\tau)-u_{2}^{-} \tau^{\prime}-\delta z_{2}\left(\tau^{\prime}\right)\right.} \\
& =\iint e^{i k \cdot u_{1}^{-} \tau} d \tau e^{-i k \cdot u_{2}^{-} \tau^{\prime}} d \tau^{\prime}\left\{u_{2}^{-\alpha}\left[-\gamma+u_{1}^{-} \cdot \delta u_{2}+u_{2}^{-} \cdot \delta u_{1}-i \gamma k \cdot \delta z_{1}(\tau)+i \gamma k \cdot \delta z_{2}\left(\tau^{\prime}\right)\right]-\gamma \delta u_{2}{ }^{\alpha}\left(\tau^{\prime}\right)\right\}(\mathrm{B} 4)
\end{aligned}
$$

The six terms entering $F^{\alpha}(k)$ can be re-written as

$$
\begin{aligned}
F^{\alpha}(k) & =-3 \gamma u_{2}^{-\alpha}(2 \pi)^{2} \delta\left(k \cdot u_{1}^{-}\right) \delta\left(k \cdot u_{2}^{-}\right) \\
& +(2 \pi) \delta\left(k \cdot u_{1}^{-}\right) u_{2}^{-\alpha} u_{1}^{-} \cdot \int e^{-i k \cdot u_{2}^{-} \tau^{\prime}} d \tau^{\prime} \delta u_{2}\left(\tau^{\prime}\right) \\
& +(2 \pi) \delta\left(k \cdot u_{2}^{-}\right) u_{2}^{-\alpha} u_{2}^{-} \cdot \int e^{i k \cdot u_{1}^{-} \tau} d \tau \delta u_{1}(\tau) \\
& +(2 \pi) \delta\left(k \cdot u_{2}^{-}\right) \gamma u_{2}^{-\alpha} \int e^{i k \cdot u_{1}^{-} \tau-i k \cdot \delta z_{1}(\tau)} d \tau \\
+ & (2 \pi) \delta\left(k \cdot u_{1}^{-}\right) \gamma u_{2}^{-\alpha} \int e^{-i k \cdot u_{2}^{-} \tau^{\prime}+i k \cdot \delta z_{2}\left(\tau^{\prime}\right)} d \tau^{\prime} \\
- & (2 \pi) \delta\left(k \cdot u_{1}^{-}\right) \gamma \int e^{-i k \cdot u_{2}^{-} \tau^{\prime}} d \tau^{\prime} \delta u_{2}{ }^{\alpha}\left(\tau^{\prime}\right) \\
\equiv & \sum_{i=1}^{6} F_{i}^{\alpha}(k),
\end{aligned}
$$

and using Eq. (B8) one finds the corresponding contributions to $\mathcal{B}_{\mu \alpha}$,

$$
\begin{aligned}
\mathcal{B}_{\mu \alpha} & \equiv \sum_{n=1}^{6} \mathcal{B}_{\mu \alpha}^{(1)} \\
& =-16 \pi G m_{2} \int \frac{d^{4} k}{(2 \pi)^{4}} \frac{i k_{\mu} e^{i k \cdot b_{0}}}{k^{2}} \sum_{n=1}^{6} F_{n \alpha}(k),
\end{aligned}
$$

identifying the various terms contributing to $\mathcal{B}_{\mu \alpha}$.

\section{Computation of $\mathcal{B}_{\mu \alpha}^{(1)}$}

In this case

$$
\begin{aligned}
\mathcal{B}_{\mu \alpha}^{(1)}= & 48 \gamma u_{2}^{-}{ }_{\alpha} \pi G m_{2} \frac{\partial}{\partial b_{0}^{\mu}} \times \\
& \int \frac{d^{4} k}{(2 \pi)^{2}} \frac{e^{i k \cdot b_{0}}}{k^{2}} \delta\left(k \cdot u_{1}^{-}\right) \delta\left(k \cdot u_{2}^{-}\right) \\
= & 48 \gamma u_{2}^{-} \alpha \pi m_{2} \frac{1}{\sqrt{\gamma^{2}-1}} \frac{\partial}{\partial b_{0}^{\mu}} \int \frac{d k^{x} d k^{z}}{(2 \pi)^{2}} \frac{e^{i k_{x} b_{0}}}{k_{x}^{2}+k_{z}^{2}} \\
= & -24 u_{2}^{-} \alpha G m_{2} \frac{\gamma}{\sqrt{\gamma^{2}-1}} \frac{\partial}{\partial b_{0}^{\mu}} \ln b_{0},
\end{aligned}
$$

so that, using the relation $\frac{\partial}{\partial b_{0}^{\mu}} b_{0}=\frac{b_{0 \mu}}{b_{0}}$,

$$
\mathcal{B}_{\mu \alpha}^{(1)}=-24 G m_{2} \frac{\gamma}{\sqrt{\gamma^{2}-1}} \frac{b_{0 \mu}}{b_{0}^{2}} u_{2}^{-} \alpha .
$$

In particular

$$
\mathcal{B}_{x y}^{(1)}=24 G m_{2} \frac{\gamma}{b_{0}}, \quad \quad \mathcal{B}_{y x}^{(1)}=0 .
$$

\section{Computation of $\mathcal{B}_{\mu \alpha}^{(2)}$}

In this case

$$
\begin{aligned}
\mathcal{B}_{\mu \alpha}^{(2)}= & -16 \pi G m_{2} u_{2}^{-} \alpha u_{1}^{-} \cdot \int d \tau^{\prime} \delta u_{2}\left(\tau^{\prime}\right) \\
& \int \frac{d^{4} k}{(2 \pi)^{3}} \frac{i k_{\mu} e^{i k \cdot\left(b_{0}-u_{2}^{-} \tau^{\prime}\right)}}{k^{2}} \delta\left(k^{0}\right) .
\end{aligned}
$$


Introducing the vectors

$$
\xi=b_{0}-u_{2}^{-} \tau^{\prime}, \quad \eta=P\left(u_{1}^{-}\right) \xi=b_{0}+\sqrt{\gamma^{2}-1} \tau^{\prime} \partial_{y} .
$$

and proceeding as before one easily finds then

$$
\mathcal{B}_{x y}^{(2)}=-2 \pi G^{2} m_{1} m_{2} \frac{\gamma\left(2 \gamma^{2}-3\right)}{b_{0}^{2}}, \quad \mathcal{B}_{y x}^{(2)}=0 .
$$

\section{Computation of $\mathcal{B}_{\mu \alpha}^{(3)}$}

In this case

$$
\begin{aligned}
\mathcal{B}_{\mu \alpha}^{(3)}= & -16 \pi G m_{2} u_{2}^{-} \alpha u_{2}^{-} \cdot \int d \tau \delta u_{1}(\tau) \\
& \int \frac{d^{4} k}{(2 \pi)^{3}} \frac{i k_{\mu} e^{i k \cdot\left(b_{0}+u_{1}^{-} \tau\right)}}{k^{2}} \delta\left(k \cdot u_{2}^{-}\right) \\
= & -16 \pi G m_{2} u_{2}^{-} \alpha u_{2}^{-} \cdot \int d \tau \delta u_{1}(\tau) \\
& \int \frac{d^{4} k}{(2 \pi)^{3}} \frac{i\left[P\left(u_{2}^{-}\right) k\right]_{\mu} e^{i P\left(u_{2}^{-}\right) k \cdot\left(b_{0}+u_{1}^{-} \tau\right)}}{\left|P\left(u_{2}^{-}\right) k\right|^{2}} \\
& \delta\left(\gamma k^{0}+\sqrt{\gamma^{2}-1} k^{y}\right),
\end{aligned}
$$

where we have replaced $k$ by $P\left(u_{2}^{-}\right) k$ because of the condition $u_{2} \cdot k=0$. The various quantities involved here reduce to

$$
\begin{aligned}
P\left(u_{2}^{-}\right) k & =k^{x} \partial_{x}+\left(\gamma^{-1} k^{y}\right) e\left(u_{2}^{-}\right)_{2}+k^{z} \partial_{z} \\
\left|P\left(u_{2}^{-}\right) k\right|^{2} & =\left(k^{x}\right)^{2}+\left(\gamma^{-1} k^{y}\right)^{2}+\left(k^{z}\right)^{2} \\
P\left(u_{2}^{-}\right) k \cdot\left(b_{0}+u_{1}^{-} \tau\right) & =k^{x} b_{0}+\frac{\sqrt{\gamma^{2}-1}}{\gamma} k^{y} \tau, \quad \text { (B15 }
\end{aligned}
$$

and defining

$$
\xi=b_{0}+u_{1}^{-} \tau, \quad \eta=P\left(u_{2}^{-}\right) \xi,
$$

we find then

$$
\mathcal{B}_{x y}^{(3)}=-2 \pi G^{2} m^{\prime 2} \frac{\gamma\left(2 \gamma^{2}-3\right)}{b_{0}^{2}}, \quad \mathcal{B}_{y x}^{(3)}=0
$$

\section{Computation of $\mathcal{B}_{\mu \alpha}^{(4)}$}

In this case we introduce the vector

$$
\begin{aligned}
\xi & =b_{0}+u_{1}^{-} \tau-\delta z_{1}(\tau) \\
{\left[P\left(u_{2}^{-}\right) k\right] \cdot \xi } & =k^{x}\left(b_{0}-\delta z_{1}^{x}\right) \\
& +\left(\gamma^{-1} k^{y}\right)\left[\sqrt{\gamma^{2}-1} \tau-\gamma \delta z_{1}^{y}\right]
\end{aligned}
$$

with

$$
P\left(u_{2}^{-}\right) k=k^{x} \partial_{x}+\left(\gamma^{-1} k^{y}\right) e\left(u_{2}^{-}\right)_{2}+k^{z} \partial_{z} .
$$

Its projection orthogonal to $u_{2}^{-}$is the four-vector

$$
\eta=P\left(u_{2}^{-}\right) \xi
$$

(not to be confused with the Minkowski metric) which reads

$$
\begin{aligned}
\eta & \equiv P\left(u_{2}^{-}\right) \xi \\
& =\left(b_{0}-\delta z_{1}^{x}\right) \partial_{x}+\left[\sqrt{\gamma^{2}-1} \tau-\gamma \delta z_{1}^{y}\right]\left(\gamma \partial_{y}-\sqrt{\gamma^{2}-1} \partial_{t}\right) \\
& =\left(b_{0}-\delta z_{1}^{x}\right) \partial_{x}+\left[\sqrt{\gamma^{2}-1} \tau-\gamma \delta z_{1}^{y}\right] e\left(u_{2}^{-}\right)_{2} \\
& =\eta^{x} \partial_{x}+\eta^{y} e\left(u_{2}^{-}\right)_{2} .
\end{aligned}
$$

Therefore

$$
\begin{aligned}
\mathcal{B}_{\mu \alpha}^{(4)} & =-16 \pi G m_{2} \gamma u_{2}^{-} \alpha \int d \tau \int \frac{d^{4} k}{(2 \pi)^{3}} \frac{i k_{\mu} e^{i k \cdot b_{0}}}{k^{2}} \delta\left(k \cdot u_{2}^{-}\right) e^{i k \cdot\left(u_{1}^{-} \tau-\delta z_{1}(\tau)\right)} \\
& =-16 \pi G m_{2} \gamma u_{2}^{-} \alpha \int d \tau \int \frac{d^{4} k}{(2 \pi)^{3}} \frac{i\left[P\left(u_{2}^{-}\right) k\right]_{\mu} e^{i P\left(u_{2}^{-}\right) k \cdot\left(b_{0}+u_{1}^{-} \tau-\delta z_{1}(\tau)\right.}}{\left|P\left(u_{2}^{-}\right) k\right|^{2}} \delta\left(k \cdot u_{2}^{-}\right) \\
& =4 G m_{2} \gamma u_{2}^{-} \alpha \int d \tau \frac{\eta_{\mu}}{\left[\left(b_{0}-\delta z_{1}^{x}\right)^{2}+\left(\sqrt{\gamma^{2}-1} \tau-\gamma \delta z_{1}^{y}\right)^{2}\right]^{3 / 2}}
\end{aligned}
$$

where, we recall

$$
\eta_{x}=b_{0}-\delta z_{1}^{x}, \quad \eta_{y}=\sqrt{\gamma^{2}-1} \tau-\gamma \delta z_{1}^{y} .
$$

In particular

$$
\mathcal{B}_{x y}^{(4)}=-8 G m^{\prime} \frac{\gamma}{b_{0}}-4 \frac{\gamma}{b_{0}^{2}} m^{\prime 2} G^{2}\left(-\pi \frac{2 \gamma^{2}+1}{2}+2 \frac{2 \gamma^{2}-1}{\gamma^{2}-1}\right), \quad \mathcal{B}_{y x}^{(4)}=0
$$




\section{Computation of $\mathcal{B}_{\mu \alpha}^{(5)}$}

Let us introduce the vector

$$
\xi=b_{0}-u_{2}^{-} \tau^{\prime}+\delta z_{2}\left(\tau^{\prime}\right), \quad\left[P\left(u_{1}^{-}\right) k\right] \cdot \xi=k^{x}\left(b_{0}+\delta z_{2}{ }^{x}\right)+k^{y}\left(\sqrt{\gamma^{2}-1} \tau^{\prime}+\delta z_{2}^{y}\right)
$$

such that

$$
\eta=P\left(u_{1}^{-}\right) \xi=\left(b_{0}+\delta z_{2}^{x}\right) \partial_{x}+\left(\sqrt{\gamma^{2}-1} \tau^{\prime}+\delta z_{2}{ }^{y}\right) \partial_{y}
$$

We find

$$
\begin{aligned}
\mathcal{B}_{\mu \alpha}^{(5)} & =-16 \pi G m_{2} \gamma u_{2}^{-} \alpha \int d \tau^{\prime} \int \frac{d^{3} k}{(2 \pi)^{3}} \frac{i\left(P\left(u_{1}^{-}\right) k\right)_{\mu} e^{i \vec{k} \cdot\left(b_{0}-P\left(u_{1}^{-}\right) u_{2}^{-} \tau^{\prime}+P\left(u_{-}\right) \delta z_{2}\left(\tau^{\prime}\right)\right)}}{\vec{k}^{2}} \\
& =-4 G m_{2} \gamma u_{2}^{-} \alpha \int d \tau^{\prime} \frac{\partial}{\partial \eta^{\mu}} \frac{1}{\left.\mid b_{0}-P\left(u_{1}^{-}\right) u_{2}^{-} \tau^{\prime}+P\left(u_{1}^{-}\right) \delta z_{2}\left(\tau^{\prime}\right)\right) \mid} \\
& =4 G m_{2} \gamma u_{2}^{-} \alpha \int d \tau^{\prime} \frac{\eta_{\mu}}{\left[\left(b_{0}+\delta z_{2}\left(\tau^{\prime}\right)^{x}\right)^{2}+\left(\sqrt{\gamma^{2}-1} \tau^{\prime}+\delta z_{2}\left(\tau^{\prime}\right)^{y}\right)^{2}\right]^{3 / 2}} .
\end{aligned}
$$

In particular

$$
\mathcal{B}_{x y}^{(5)}=-8 G m_{2} \frac{\gamma}{b_{0}}-4 G^{2} m_{1} m_{2}\left(-\frac{1}{2}\left(2 \gamma^{2}+1\right) \pi+2 \frac{2 \gamma^{2}-1}{\gamma^{2}-1}\right) \frac{\gamma}{b_{0}^{2}}, \quad \mathcal{B}_{y x}^{(5)}=0
$$

\section{Computation of $\mathcal{B}_{\mu \alpha}^{(6)}$}

Let us introduce the vectors

$$
\xi=b_{0}-u_{2}^{-} \tau^{\prime}, \quad \eta=P\left(u_{1}^{-}\right) \xi=b_{0}+\sqrt{\gamma^{2}-1} \tau^{\prime} \partial_{y} .
$$

We have then

$$
\begin{aligned}
\mathcal{B}_{\mu \alpha}^{(6)} & =+16 \pi G m_{2} \gamma \int d \tau^{\prime} \delta u_{2}\left(\tau^{\prime}\right)_{\alpha} \int \frac{d^{4} k}{(2 \pi)^{3}} \frac{i k_{\mu} e^{i k \cdot b_{0}}}{k^{2}} \delta\left(k \cdot u_{1}^{-}\right) e^{-i k \cdot u_{2}^{-} \tau^{\prime}} \\
& =+16 \pi G m_{2} \gamma \int d \tau^{\prime} \delta u_{2}\left(\tau^{\prime}\right)_{\alpha} \frac{\partial}{\partial \eta^{\mu}} \int \frac{d^{3} k}{(2 \pi)^{3}} \frac{e^{i \vec{k} \cdot\left(b_{0}-P\left(u_{1}^{-}\right) u_{2}^{-} \tau^{\prime}\right)}}{\vec{k}^{2}} \\
& =+4 G m_{2} \gamma \int d \tau^{\prime} \delta u_{2}\left(\tau^{\prime}\right)_{\alpha} \frac{-\eta_{\mu}}{D\left(\tau^{\prime}\right)^{3}}
\end{aligned}
$$

In particular

$$
\mathcal{B}_{x y}^{(6)}=-2 G^{2} m_{1} m_{2} \pi \frac{\gamma^{2}\left(2 \gamma^{2}-3\right)}{\left(\gamma^{2}-1\right)} \frac{\gamma}{b_{0}^{2}}, \quad \mathcal{B}_{y x}^{(6)}=-2 G^{2} m_{1} m_{2} \pi \frac{\left(2 \gamma^{2}-1\right)}{\gamma^{2}-1} \frac{\gamma}{b_{0}^{2}}
$$

\section{Appendix C: The general case: computation of $\mathcal{C}_{\mu \alpha}$}

Let us recall

$$
\begin{aligned}
\mathcal{C}_{\mu \beta} & =16 \pi G m_{2} \int u_{1 \beta}(\tau) d \tau \int \frac{d^{4} k}{(2 \pi)^{4}} \frac{i k_{\mu}}{k^{2}} \int d \tau^{\prime} e^{i k \cdot b_{0}} e^{i\left(u_{1}^{-} \tau+\delta z_{1}(\tau)-u_{2}^{-} \tau^{\prime}-\delta z_{2}\left(\tau^{\prime}\right)\right)} \\
& =16 \pi G m_{2} \iint d \tau d \tau^{\prime} \int \frac{d^{4} k}{(2 \pi)^{4}} \frac{i k_{\mu} e^{i k \cdot b_{0}}}{k^{2}} e^{i k \cdot u_{1}^{-} \tau} e^{-i k \cdot u_{2}^{-} \tau^{\prime}}\left[u_{1}^{-} \beta\left(e^{i k \cdot \delta z_{1}(\tau)}+e^{-i k \cdot \delta z_{2}\left(\tau^{\prime}\right)}-1\right)+\delta u_{1 \beta}(\tau)\right]
\end{aligned}
$$


We will compute separately the following four terms

$$
\begin{aligned}
& \mathcal{C}_{\mu \beta}^{(1)}=16 \pi G m_{2} u_{1 \beta}^{-} \iint d \tau d \tau^{\prime} \int \frac{d^{4} k}{(2 \pi)^{4}} \frac{i k_{\mu} e^{i k \cdot b_{0}}}{k^{2}} e^{i k \cdot u_{1}^{-} \tau} e^{-i k \cdot u_{2}^{-} \tau^{\prime}} e^{i k \cdot \delta z_{1}(\tau)} \\
& \mathcal{C}_{\mu \beta}^{(2)}=16 \pi G m_{2} u_{1}^{-} \beta \iint d \tau d \tau^{\prime} \int \frac{d^{4} k}{(2 \pi)^{4}} \frac{i k_{\mu} e^{i k \cdot b_{0}}}{k^{2}} e^{i k \cdot u_{1}^{-} \tau} e^{-i k \cdot u_{2}^{-} \tau^{\prime}} e^{-i k \cdot \delta z_{2}\left(\tau^{\prime}\right)} \\
& \mathcal{C}_{\mu \beta}^{(3)}=-16 \pi G m_{2} u_{1 \beta}^{-} \iint d \tau d \tau^{\prime} \int \frac{d^{4} k}{(2 \pi)^{4}} \frac{i k_{\mu} e^{i k \cdot b_{0}}}{k^{2}} e^{i k \cdot u_{1}^{-} \tau} e^{-i k \cdot u_{2}^{-} \tau^{\prime}} \\
& \mathcal{C}_{\mu \beta}^{(4)}=16 \pi G m_{2} \iint d \tau d \tau^{\prime} \int \frac{d^{4} k}{(2 \pi)^{4}} \frac{i k_{\mu} e^{i k \cdot b_{0}}}{k^{2}} e^{i k \cdot u_{1}^{-} \tau} e^{-i k \cdot u_{2}^{-} \tau^{\prime}} \delta u_{1 \beta}(\tau) .
\end{aligned}
$$

\section{Computation of $\mathcal{C}_{\mu \alpha}^{(1)}$}

We find

$$
\begin{aligned}
\mathcal{C}_{\mu \beta}^{(1)} & =16 \pi G m_{2} u_{1}^{-} \beta \iint d \tau d \tau^{\prime} \int \frac{d^{4} k}{(2 \pi)^{4}} \frac{i k_{\mu} e^{i k \cdot b_{0}}}{k^{2}} e^{i k \cdot u_{1}^{-} \tau} e^{-i k \cdot u_{2}^{-} \tau^{\prime}} e^{i k \cdot \delta z_{1}(\tau)} \\
& =16 \pi G m_{2} u_{1}^{-} \beta \int d \tau \int \frac{d^{4} k}{(2 \pi)^{3}} \frac{i k_{\mu} e^{i k \cdot b_{0}}}{k^{2}} e^{i k \cdot u_{1}^{-} \tau} e^{i k \cdot \delta z_{1}(\tau)} \delta\left(k \cdot u_{2}^{-}\right) \\
& =16 \pi G m_{2} u_{1}^{-} \beta \int d \tau \int \frac{d k^{x} d\left(\gamma^{-1}\right) k^{y} d k^{z}}{(2 \pi)^{3}} \frac{i\left[P\left(u_{2}^{-}\right) k\right]_{\mu} e^{i P\left(u_{2}^{-}\right) k \cdot\left(b_{0}+u_{1}^{-} \tau+\delta z_{1}(\tau)\right)}}{\left|P\left(u_{2}^{-}\right) k\right|^{2}}
\end{aligned}
$$

Let us introduce the vectors

$$
\xi=b_{0}+u_{1}^{-} \tau+\delta z_{1}(\tau), \quad \eta=P\left(u_{2}^{-}\right) \xi
$$

such that

$$
\begin{aligned}
P\left(u_{2}^{-}\right) k \cdot \xi & =k^{x}\left(b_{0}+\delta z_{1}^{x}\right)+\left(\gamma^{-1} k^{y}\right)\left[\sqrt{\gamma^{2}-1} \tau+\gamma \delta z_{1}^{y}\right] \\
\eta & =\left(b_{0}+\delta z_{1}^{x}\right) \partial_{x}+\left[\sqrt{\gamma^{2}-1} \tau+\gamma \delta z_{1}^{y}\right] e\left(u_{2}^{-}\right)_{2} .
\end{aligned}
$$

A straightforward computation shows that

$$
\mathcal{C}_{x y}^{(1)}=\mathcal{C}_{y x}^{(1)}=0
$$

\section{Computation of $\mathcal{C}_{\mu \alpha}^{(2)}$}

$$
\begin{aligned}
\mathcal{C}_{\mu \beta}^{(2)} & =16 \pi G m_{2} u_{1}^{-} \beta \iint d \tau d \tau^{\prime} \int \frac{d^{4} k}{(2 \pi)^{4}} \frac{i k_{\mu} e^{i k \cdot b_{0}}}{k^{2}} e^{i k \cdot u_{1}^{-} \tau} e^{-i k \cdot u_{2}^{-} \tau^{\prime}} e^{-i k \cdot \delta z_{2}\left(\tau^{\prime}\right)} \\
& =16 \pi G m_{2} u_{1}^{-} \beta \int d \tau^{\prime} \int \frac{d^{4} k}{(2 \pi)^{3}} \frac{i k_{\mu} e^{i k \cdot b_{0}}}{k^{2}} \delta\left(k \cdot u_{1}^{-}\right) e^{-i k \cdot u_{2}^{-} \tau^{\prime}} e^{-i k \cdot \delta z_{2}\left(\tau^{\prime}\right)} \\
& =16 \pi G m_{2} u_{1}^{-} \beta \int d \tau^{\prime} \int \frac{d^{3} k}{(2 \pi)^{3}} \frac{i\left[P\left(u_{1}^{-}\right) k\right]_{\mu} e^{i P\left(u_{1}^{-}\right) k \cdot\left(b_{0}-u_{2}^{-} \tau^{\prime}-\delta z_{2}\left(\tau^{\prime}\right)\right)}}{\left|P\left(u_{1}^{-}\right) k\right|^{2}}
\end{aligned}
$$

Let us introduce the vectors

$$
\xi=b_{0}-u_{2}^{-}-\tau^{\prime}-\delta z_{2}\left(\tau^{\prime}\right), \quad \eta=P\left(u_{1}^{-}\right) \xi
$$

such that

$$
\begin{aligned}
P\left(u_{1}^{-}\right) k \cdot \xi & =k^{x}\left(b_{0}-\delta z_{2}{ }^{x}\right)+k^{y}\left[\sqrt{\gamma^{2}-1} \tau^{\prime}-\delta z_{2}{ }^{y}\right] \\
\eta & =\left(b_{0}-\delta z_{2}{ }^{x}\right) \partial_{x}+\left[\sqrt{\gamma^{2}-1} \tau^{\prime}-\delta z_{2}{ }^{y}\right] \partial_{y} .
\end{aligned}
$$

Proceeding as before one finds

$$
\mathcal{C}_{x y}^{(2)}=\mathcal{C}_{y x}^{(2)}=0
$$




\section{Computation of $\mathcal{C}_{\mu \alpha}^{(3)}$}

$$
\begin{aligned}
\mathcal{C}_{\mu \beta}^{(3)} & =-16 \pi G m_{2} u_{1 \beta}^{-} \frac{\partial}{\partial b_{0}^{\mu}} \iint d \tau d \tau^{\prime} \int \frac{d^{4} k}{(2 \pi)^{4}} \frac{e^{i k \cdot b_{0}}}{k^{2}} e^{i k \cdot u_{1}^{-} \tau} e^{-i k \cdot u_{2}^{-} \tau^{\prime}} \\
& =-16 \pi G m_{2} u_{1}^{-} \beta \frac{\partial}{\partial b_{0}^{\mu}} \int \frac{d^{4} k}{(2 \pi)^{2}} \frac{e^{i k \cdot b_{0}}}{k^{2}} \delta\left(k \cdot u_{1}^{-}\right) \delta\left(k \cdot u_{2}^{-}\right) \\
& =8 G m_{2} u_{1}^{-}{ }^{-} \frac{b_{0 \mu}}{b_{0}^{2}}
\end{aligned}
$$

Also in this case $\mathcal{C}_{x y}^{(3)}=\mathcal{C}_{y x}^{(3)}=0$.

\section{Computation of $\mathcal{C}_{\mu \alpha}^{(4)}$}

We have

$$
\begin{aligned}
\mathcal{C}_{\mu \beta}^{(4)} & =16 \pi G m_{2} \iint d \tau d \tau^{\prime} \int \frac{d^{4} k}{(2 \pi)^{4}} \frac{i k_{\mu} e^{i k \cdot b_{0}}}{k^{2}} e^{i k \cdot u_{1}^{-} \tau} e^{-i k \cdot u_{2}^{-} \tau^{\prime}} \delta u_{1 \beta}(\tau) \\
& =16 \pi G m_{2} \int d \tau \delta u_{1 \beta}(\tau) \int \frac{d^{4} k}{(2 \pi)^{3}} \frac{i k_{\mu} e^{i k \cdot\left(b_{0}+u_{1}^{-} \tau\right)}}{k^{2}} \delta\left(k \cdot u_{2}^{-}\right)
\end{aligned}
$$

Let

$$
\xi=b_{0}+u_{1}^{-} \tau, \quad \eta=P\left(u_{2}^{-}\right) \xi=b_{0}+\tau \sqrt{\gamma^{2}-1} e\left(u_{2}^{-}\right)_{2}
$$

We find

$$
\begin{aligned}
\mathcal{C}_{\mu \beta}^{(4)} & =16 \pi G m_{2} \int d \tau \delta u_{\beta}(\tau) \frac{\partial}{\partial \eta^{\mu}} \int \frac{d^{4} k}{(2 \pi)^{3}} \frac{e^{i P\left(u_{2}^{-}\right) k \cdot\left(b_{0}+u_{1}^{-} \tau\right)}}{\left|P\left(u_{2}^{-}\right) k\right|^{2}} \frac{1}{\gamma} \delta\left(k^{0}+\gamma^{-1} \sqrt{\gamma^{2}-1} k^{y}\right) \\
& =16 \pi G m_{2} \int d \tau \delta u_{1 \beta}(\tau) \frac{\partial}{\partial \eta^{\mu}} \int \frac{d k^{x} d\left(\gamma^{-1} k^{y}\right) d k^{z}}{(2 \pi)^{3}} \frac{\left.e^{i\left(k^{x} b+\frac{\sqrt{\gamma^{2}-1}}{\gamma}\right.} k^{y} \tau\right)}{\left(k^{x}\right)^{2}+\left(\gamma^{-1} k^{y}\right)^{2}+\left(k^{z}\right)^{2}} \\
& =4 G m_{2} \int d \tau \delta u_{1 \beta}(\tau) \frac{\partial}{\partial \eta^{\mu}} \frac{1}{\sqrt{b_{0}^{2}+\left(\gamma^{2}-1\right) \tau^{2}}} \\
& =-4 G m_{2} \int d \tau \delta u_{1 \beta}(\tau) \frac{\eta_{\mu}}{\left[b_{0}^{2}+\left(\gamma^{2}-1\right) \tau^{2}\right]^{3 / 2}} \\
& =\mathcal{C}_{\mu x}^{(4)} \delta_{\beta}^{x}+\mathcal{C}_{\mu y}^{(4)} \delta_{\beta}^{y} .
\end{aligned}
$$

In particular

$$
\mathcal{C}_{x y}^{(4)}=2 G^{2} m_{2}{ }^{2} \pi \frac{\gamma\left(2 \gamma^{2}-3\right)}{\gamma^{2}-1} \frac{1}{b_{0}^{2}}, \quad \mathcal{C}_{y x}^{(4)}=2 G^{2} m_{2}{ }^{2} \pi \frac{\gamma\left(2 \gamma^{2}-1\right)}{\left(\gamma^{2}-1\right)} \frac{1}{b_{0}^{2}}
$$

We can now proceed summing up all the various contributions (generated by the the first order in $\mathrm{h}$ terms of the metric) entering $\Lambda_{12}^{1}$, i.e., recalling Eq. A10

$$
\Lambda_{(1)^{2}}^{1}=\frac{1}{2}\left(\mathcal{B}_{x y}-\mathcal{B}_{y x}\right)-\frac{1}{4}\left(\mathcal{C}_{x y}-\mathcal{C}_{y x}\right)=\mathcal{B}_{[x y]}-\frac{1}{2} \mathcal{C}_{[x y]} \equiv \Lambda_{\mathcal{B}}{ }^{x}{ }_{y}+\Lambda_{\mathcal{C}}{ }^{x} y
$$


with $\Lambda_{\mathcal{B}}{ }^{x} y=\sum_{n=1}^{6} \mathcal{B}_{[x y]}^{(n)}$ and $\Lambda_{\mathcal{C}}{ }^{x} y=-\frac{1}{2} \sum_{n=1}^{4} \mathcal{C}_{[x y]}^{(n)}$. Summing up the various contributions to $\Lambda_{\mathcal{B}}{ }^{x} y$ we find:

$$
\begin{array}{lr}
\mathcal{B}_{[x y]}^{(1)}=+12 G m_{2} \frac{\gamma}{b_{0}} & +0 \\
\mathcal{B}_{[x y]}^{(2)}=0 & -\pi G^{2} m_{1} m_{2} \frac{\gamma\left(2 \gamma^{2}-3\right)}{b_{0}^{2}} \\
\mathcal{B}_{[x y]}^{(3)}=0 & -\pi G^{2} m_{2} \frac{\gamma\left(2 \gamma^{2}-3\right)}{b_{0}^{2}} \\
\mathcal{B}_{[x y]}^{(4)}=-4 G m_{2} \frac{\gamma}{b_{0}} & -2 G^{2} m_{2}^{2}\left(-\frac{1}{2} \pi\left(2 \gamma^{2}+1\right)+\frac{2\left(2 \gamma^{2}-1\right)}{\gamma^{2}-1}\right) \frac{\gamma}{b_{0}^{2}} \\
\mathcal{B}_{[x y]}^{(5)}=-4 G m_{2} \frac{\gamma}{b_{0}} & -2 G^{2} m_{1} m_{2}\left(-\frac{1}{2} \pi\left(2 \gamma^{2}+1\right)+\frac{2\left(2 \gamma^{2}-1\right)}{\gamma^{2}-1}\right) \frac{\gamma}{b_{0}^{2}} \\
\mathcal{B}_{[x y]}^{(6)}=0 & -\pi G^{2} m_{1} m_{2} \frac{\gamma^{3}\left(2 \gamma^{2}-3\right)}{\gamma^{2}-1} \frac{1}{b_{0}^{2}}+\pi G^{2} m_{1} m_{2} \frac{\left(2 \gamma^{2}-1\right)}{\gamma^{2}-1} \frac{\gamma}{b_{0}^{2}}
\end{array}
$$

Similarly, summing the various contributions to $-2 \Lambda_{\mathcal{C}^{x} y}=\sum_{n=1}^{4} \mathcal{C}_{[x y]}^{(n)}$ we find:

$$
\begin{array}{lrr}
\mathcal{C}_{[x y]}^{(1)}=0 & +0 \\
\mathcal{C}_{[x y]}^{(2)}=0 & +0 \\
\mathcal{C}_{[x y]}^{(3)}=0 & +0 \\
\mathcal{C}_{[x y]}^{(4)}=0 & +\pi G^{2} m_{2} \frac{2}{2} \frac{\gamma\left(2 \gamma^{2}-3\right)}{\gamma^{2}-1} \frac{1}{b_{0}^{2}}-G^{2} m_{2}{ }^{2} \pi \frac{\gamma\left(2 \gamma^{2}-1\right)}{\left(\gamma^{2}-1\right)} \frac{1}{b_{0}^{2}}
\end{array}
$$

Including the quadratic term

$$
\int_{-\infty}^{\infty} d \tau \int_{-\infty}^{\tau} d \tau^{\prime} \omega_{1}(\tau)^{x}{ }_{\beta} \omega_{1}\left(\tau^{\prime}\right)^{\beta}{ }_{y}=-\frac{\pi G^{2} m_{2}^{2}}{2} \frac{\gamma\left(4 \gamma^{2}-3\right)}{b_{0}^{2}\left(\gamma^{2}-1\right)}
$$

and comparing with our previous computations (those using only the 1PM metric), namely

$$
\begin{aligned}
\Lambda_{(1) 2}^{1} & =\frac{4 G m^{\prime} \gamma}{b_{0}}+\frac{\gamma G^{2} m_{2}^{2}\left(2 \gamma^{2}+1\right) \pi}{b_{0}^{2}}-4 G^{2} m^{\prime 2} \gamma \frac{\left(2 \gamma^{2}-1\right)}{b_{0}^{2}\left(\gamma^{2}-1\right)} \\
& -2 G^{2} m_{1} m_{2} \pi \frac{\gamma\left(\gamma^{2}-2\right)\left(2 \gamma^{2}-1\right)}{b_{0}^{2}\left(\gamma^{2}-1\right)} \\
& +G^{2} m_{1} m_{2} \pi \frac{\gamma\left(2 \gamma^{2}+1\right)}{b_{0}^{2}}-4 G^{2} m m^{\prime} \frac{\gamma\left(2 \gamma^{2}-1\right)}{b_{0}^{2}\left(\gamma^{2}-1\right)} \\
& +G^{2} m_{2}{ }^{2} \pi \frac{\gamma\left(2 \gamma^{2}-1\right)}{2 b_{0}^{2}\left(\gamma^{2}-1\right)} \\
& -G^{2} m_{2}{ }^{2} \pi \frac{\gamma\left(2 \gamma^{2}-1\right)\left(2 \gamma^{2}-3\right)}{2 b_{0}^{2}\left(\gamma^{2}-1\right)} \\
& -\frac{G^{2} m_{2}{ }^{2} \pi}{2} \frac{\gamma\left(4 \gamma^{2}-3\right)}{b_{0}^{2}\left(\gamma^{2}-1\right)}
\end{aligned}
$$

we find complete agreement.

\section{Appendix D: The impact parameter at 1PM order}

The explicit calculation showing the relation between $b$ and $b_{0}$ uses

$$
L_{\lambda}=U^{\mathrm{as} \sigma} \epsilon_{\sigma \mu \nu \lambda}\left(m_{1} z_{1}^{\mu}(\tau) u_{1}^{\nu}(\tau)+m_{2} z_{2}{ }^{\mu}\left(\tau^{\prime}\right) u_{2}{ }^{\nu}\left(\tau^{\prime}\right)\right)
$$

where $U^{\text {as }}$ has been defined in Eq. (3.3). We find $L_{\lambda}=$ $L \delta_{\lambda}^{z}=L_{0}+G \delta L$ with

$$
\begin{aligned}
L_{0}= & m b \sinh \alpha \\
\delta L= & {\left[m_{2} \sqrt{\gamma^{2}-1}\left(\delta u_{2}{ }^{x} \tau^{\prime}-\delta z_{2}{ }^{x}\left(\tau^{\prime}\right)\right)\right.} \\
& \left.+m_{1} b \delta u_{1}^{y}(\tau)\right] \cosh \alpha \\
& -\left[m_{2} \gamma\left(\delta u_{2}{ }^{x} \tau^{\prime}-\delta z_{2}{ }^{x}\left(\tau^{\prime}\right)\right)\right. \\
& \left.+m_{1}\left(\delta u_{1}^{x} \tau-\delta z_{1}^{x}(\tau)\right)\right] \sinh \alpha
\end{aligned}
$$


In this expression $\tau$ must be replaced by its functional link with $\tau^{\prime}$ implied by the definition of center-of-mass frame:

$$
U^{\mathrm{as}} \cdot\left(z_{2}\left(\tau^{\prime}\right)-z_{1}(\tau)\right)=0
$$

that is

$$
\tau=\left(\gamma_{0}-\sqrt{\gamma^{2}-1} \tanh \alpha\right) \tau^{\prime}+G \delta \tau^{\prime} .
$$

We see that it is enough to know the relation $\tau$ vs $\tau^{\prime}$ at the zeroth order in $G$, which implies

$$
\begin{aligned}
\delta L= & m_{1} \sinh \alpha\left[\left(\delta u_{2}^{x}\left(\tau^{\prime}\right) \tau^{\prime}-\delta z_{2}^{x}\left(\tau^{\prime}\right)\right)\right. \\
& \left.-\left(\delta u_{1}^{x}(\tau) \tau-\delta z_{1}^{x}(\tau)\right)\right] \\
& +m_{1} b \cosh \alpha \delta u_{1}^{y}(\tau)
\end{aligned}
$$

Taking into account that for $\tau \rightarrow+\infty$

$$
\frac{S(\tau)}{D(\tau)} \tau-\frac{S-1}{\sqrt{\gamma^{2}-1}}=\frac{1}{\sqrt{\gamma^{2}-1}}+O\left(\frac{1}{\tau}\right)
$$

we find

$$
\delta u_{2}^{x}\left(\tau^{\prime}\right) \tau^{\prime}-\delta z_{2}{ }^{x}\left(\tau^{\prime}\right)= \begin{cases}+\infty & -G m_{2} \frac{1-2 \gamma^{2}}{\gamma^{2}-1} \\ -\infty & -G m_{1} \frac{1-2 \gamma^{2}}{\gamma^{2}-1}\end{cases}
$$

and

$$
\delta u_{1}^{x}(\tau) \tau-\delta z_{1}^{x}(\tau)= \begin{cases}+\infty & G m_{1} \frac{1-2 \gamma^{2}}{\gamma^{2}-1} \\ -\infty & +G m_{2} \frac{1-2 \gamma^{2}}{\gamma^{2}-1}\end{cases}
$$

Moreover,

$$
\lim _{\tau= \pm \infty} \delta u^{y}(\tau)=0
$$

Taking the limit $\tau^{\prime} \rightarrow-\infty$ gives

$$
\begin{aligned}
L_{-} & =m_{1} b_{0} \sinh \alpha+G \frac{\left(2 \gamma^{2}-1\right)}{\gamma^{2}-1} m_{1}\left(m_{1}+m_{2}\right) \sinh \alpha \\
& =m_{1} \sinh \alpha\left(b_{0}+G \delta b\right),
\end{aligned}
$$

and hence

$$
\delta b=\frac{\left(2 \gamma^{2}-1\right)}{\gamma^{2}-1}\left(m_{1}+m_{2}\right) .
$$

Taking the limit $\tau^{\prime} \rightarrow+\infty$ gives exactly the same limit, i.e.,

$$
L_{+}=m_{1} b_{0} \sinh \alpha+G \frac{\left(2 \gamma^{2}-1\right)}{\gamma^{2}-1} m_{1}\left(m_{1}+m_{2}\right) \sinh \alpha,
$$

in agreement with previous results.

\section{Acknowledgments}

D.B. thanks the International Center for Relativistic Astrophysics Network (ICRANet) and the Italian Istituto Nazionale di Fisica Nucleare (INFN) for partial support and the Institut des Hautes Etudes Scientifiques (IHES) for warm hospitality at various stages during the development of the present project.
[1] A. Buonanno and T. Damour, "Effective one-body approach to general relativistic two-body dynamics," Phys. Rev. D 59, 084006 (1999) gr-qc/9811091.

[2] A. Buonanno and T. Damour, "Transition from inspiral to plunge in binary black hole coalescences," Phys. Rev. D 62, 064015 (2000) gr-qc/0001013.

[3] T. Damour, P. Jaranowski and G. Schäfer, "On the determination of the last stable orbit for circular general relativistic binaries at the third post-Newtonian approximation," Phys. Rev. D 62, 084011 (2000) gr-qc/0005034.

[4] T. Damour, "Coalescence of two spinning black holes: an effective one-body approach," Phys. Rev. D 64, 124013 (2001) gr-qc/0103018.

[5] A. Taracchini et al., "Effective-one-body model for black-hole binaries with generic mass ratios and spins," Phys. Rev. D 89, 061502 (2014) doi:10.1103/PhysRevD.89.061502 [gr-qc]].

[6] A. Bohé et al., "Improved effective-one-body model of spinning, nonprecessing binary black holes for the era of gravitational-wave astrophysics with advanced detectors," Phys. Rev. D 95, no. 4, 044028 (2017) doi:10.1103/PhysRevD.95.044028 arXiv:1611.03703 [grqc]].
[7] B. P. Abbott et al. [LIGO Scientific and Virgo Collaborations], "Observation of Gravitational Waves from a Binary Black Hole Merger," Phys. Rev. Lett. 116, 061102 (2016) doi:10.1103/PhysRevLett.116.061102 arXiv:1602.03837 [gr-qc]].

[8] B. P. Abbott et al. [LIGO Scientific and Virgo Collaborations], "GW151226: Observation of Gravitational Waves from a 22-Solar-Mass Binary Black Hole Coalescence," Phys. Rev. Lett. 116, no. 24, 241103 (2016) doi:10.1103/PhysRevLett.116.241103 arXiv:1606.04855 [gr-qc]].

[9] B. P. Abbott et al. [LIGO Scientific and VIRGO Collaborations], "GW170104: Observation of a 50Solar-Mass Binary Black Hole Coalescence at Redshift 0.2," Phys. Rev. Lett. 118 (2017) no.22, 221101. doi:10.1103/PhysRevLett.118.221101

[10] B. P. Abbott et al. [LIGO Scientific and Virgo Collaborations], "GW170814: A Three-Detector Observation of Gravitational Waves from a Binary Black Hole Coalescence," Phys. Rev. Lett. 119, no. 14, 141101 (2017) doi:10.1103/PhysRevLett.119.141101 arXiv:1709.09660 [gr-qc]].

[11] T. Damour, B. R. Iyer and A. Nagar, "Improved resummation of post-Newtonian multipolar waveforms 
from circularized compact binaries," Phys. Rev. D 79, 064004 (2009) doi:10.1103/PhysRevD.79.064004 arXiv:0811.2069 [gr-qc]].

[12] A. Buonanno, Y. Pan, J. G. Baker, J. Centrella, B. J. Kelly, S. T. McWilliams and J. R. van Meter, "Toward faithful templates for non-spinning binary black holes using the effective-one-body approach," Phys. Rev. D 76, 104049 (2007) doi:10.1103/PhysRevD.76.104049 arXiv:0706.3732 [gr-qc]].

[13] T. Damour and A. Nagar, "Comparing EffectiveOne-Body gravitational waveforms to accurate numerical data," Phys. Rev. D 77, 024043 (2008) doi:10.1103/PhysRevD.77.024043 arXiv:0711.2628 [grqc]].

[14] T. Damour, A. Nagar, E. N. Dorband, D. Pollney and L. Rezzolla, "Faithful Effective-One-Body waveforms of equal-mass coalescing black-hole binaries," Phys. Rev. D 77, 084017 (2008) doi:10.1103/PhysRevD.77.084017 arXiv:0712.3003 [gr-qc]].

[15] S. Akcay, L. Barack, T. Damour and N. Sago, "Gravitational self-force and the effective-one-body formalism between the innermost stable circular orbit and the light ring," Phys. Rev. D 86, 104041 (2012) doi:10.1103/PhysRevD.86.104041 arXiv:1209.0964 [gr$\mathrm{qc}]]$.

[16] T. Damour, "High-energy gravitational scattering and the general relativistic two-body problem," Phys. Rev. D 97, no. 4, 044038 (2018) doi:10.1103/PhysRevD.97.044038 arXiv:1710.10599 [gr-qc]].

[17] T. Damour, "Gravitational scattering, postMinkowskian approximation and Effective One-Body theory," Phys. Rev. D 94, no. 10, 104015 (2016) doi:10.1103/PhysRevD.94.104015 arXiv:1609.00354 [gr-qc]].

[18] D. Bini and T. Damour, "Gravitational spin-orbit coupling in binary systems, post-Minkowskian approximation and effective one-body theory," Phys. Rev. D 96, no. 10, 104038 (2017) doi:10.1103/PhysRevD.96.104038 arXiv:1709.00590 [gr-qc]].

[19] J. Vines, "Scattering of two spinning black holes in post-Minkowskian gravity, to all orders in spin, and effective-one-body mappings," Class. Quant. Grav. 35, no. 8, 084002 (2018) doi:10.1088/1361-6382/aaa3a8 arXiv:1709.06016 [gr-qc]].

[20] L. Bel, T. Damour, N. Deruelle, J. Ibanez and J. Martin, "Poincaré-invariant gravitational field and equations of motion of two pointlike objects: The postlinear approximation of general relativity," Gen. Rel. Grav. 13, 963 (1981). doi:10.1007/BF00756073
We note the following misprints in this paper: Eq. C20: minus sign needed at the rhs; Eq. C36: the last two terms in the square bracket should be $\nu_{\beta} \tilde{\nu}_{\alpha}$,i.e., the tilde should be exchanged; Eq. C42: $B_{\mu}=v_{\mu}-\tilde{\nu}_{\mu}$.

[21] K. Westpfahl, "High-Speed Scattering of Charged and Uncharged Particles in General Relativity," Fortschr. Physik 33, 417 (1985). DOI: 10.1002/prop.2190330802

[22] F. J. Dyson, "The Radiation theories of Tomonaga, Schwinger, and Feynman," Phys. Rev. 75, 486 (1949). doi:10.1103/PhysRev.75.486

[23] T. Damour, "A New and Consistent Method for Classical Renormalization," Nuovo Cim. B 26, 157 (1975). doi:10.1007/BF02755544

[24] T. Damour, P. Jaranowski and G. Schaefer, "Effective one body approach to the dynamics of two spinning black holes with next-to-leading order spinorbit coupling," Phys. Rev. D 78, 024009 (2008) doi:10.1103/PhysRevD.78.024009 arXiv:0803.0915 [grqc]].

[25] T. Damour and G. Schaefer, "Higher Order Relativistic Periastron Advances and Binary Pulsars," Nuovo Cim. B 101, 127 (1988). doi:10.1007/BF02828697

[26] A. Nagar, "Effective one body Hamiltonian of two spinning black-holes with next-to-next-to-leading order spin-orbit coupling," Phys. Rev. D 84, 084028 (2011) Erratum: [Phys. Rev. D 88, no. 8, 089901 (2013)] doi:10.1103/PhysRevD.84.084028, 10.1103/PhysRevD.88.089901 arXiv:1106.4349 [gr-qc]].

[27] E. Barausse and A. Buonanno, "Extending the effectiveone-body Hamiltonian of black-hole binaries to include next-to-next-to-leading spin-orbit couplings," Phys. Rev. D 84, 104027 (2011) doi:10.1103/PhysRevD.84.104027 arXiv:1107.2904 [gr-qc]].

[28] E. Barausse, E. Racine and A. Buonanno, "Hamiltonian of a spinning test-particle in curved spacetime," Phys. Rev. D 80, 104025 (2009) Erratum: [Phys. Rev. D 85, 069904 (2012)] doi:10.1103/PhysRevD.85.069904, 10.1103/PhysRevD.80.104025 arXiv:0907.4745 [gr-qc]].

[29] E. Barausse and A. Buonanno, "An Improved effective-one-body Hamiltonian for spinning blackhole binaries," Phys. Rev. D 81, 084024 (2010) doi:10.1103/PhysRevD.81.084024 arXiv:0912.3517] [grqc]].

[30] D. Bini, T. Damour and A. Geralico, "Spin-dependent two-body interactions from gravitational self-force computations," Phys. Rev. D 92, no. 12, 124058 (2015) Erratum: [Phys. Rev. D 93, no. 10, 109902 (2016)] doi:10.1103/PhysRevD.93.109902, 10.1103/PhysRevD.92.124058 arXiv:1510.06230 [gr-qc]]. 